\title{
Absorption Spectrum of Water Vapor Between 4.5 and 13 Microns
}

\author{
W. S. Benedict, ${ }^{2}$ H. H. Claassen, ${ }^{3}$ and J. H. Shaw ${ }^{4}$
}

\begin{abstract}
The absorption spectrum of water vapor has been measured from 4.5 to 13 microns with a 3,600 line per inch replica echelette grating as the dispersing element. Various absorbing path lengths and concentrations of water vapor at atmospheric pressure were used up to 8 meters of steam near $110^{\circ} \mathrm{C}$. Almost all of the previously unreported lines that have been found are also present in the solar spectrum. A rotational analysis shows that most of the lines can be assigned either to rotational transitions or to rotation-vibration transitions of the $\nu_{2}$ fundamental of the water-vapor molecule. In addition, a few lines have also been assigned to the transitions $\left(2 \nu_{2}-\nu_{2}\right),\left(\nu_{1}-\nu_{2}\right)$, and $\left(\nu_{3}-\nu_{2}\right)$.
\end{abstract}

\section{Introduction}

Investigations of the infrared spectrum of sunlight between 7 and $13 \mu$ have shown that essentially all the structure is due to absorption by polyatomic gases that are in the earth's atmosphere. In addition to the known bands of ozone, carbon dioxide, water vapor, nitrous oxide, and methane that have been identified in this region, there is also a large number of irregularly spaced absorption lines. Some of these lines are very strong. In solar spectra taken at Columbus, it has been observed that most of their intensities are dependent on the amount of water vapor in the atmosphere. One of us (W.S.B.) had previously found that many of these lines observed in the grating map of the solar spectrum published by Adel $[1]^{5}$ could be predicted from the known energy levels of the water-vapor molecule. To obtain a positive identification of these lines, it was thought desirable to observe them in a laboratory spectrum. If these lines are due to water vapor, most of them must originate from the higher rotational energy levels of the molecule, which have a low population at room temperature. Thus, in order to observe them in the laboratory, an absorbing layer giving an optical path length approaching that of the water vapor in the atmosphere is required. In this work a long path length was obtained by using a multiple-reflection cell similar to that described by J. U. White [2]. From this laboratory spectrum it has been possible to identify many lines in the solar spectrum with watervapor lines and also to evaluate rotational energy levels of the $\nu_{2}$ band and of the pure-rotation band, which are in good agreement with levels derived from other observations.

\section{Apparatus and Experimental Procedure}

The absorption cell used in this investigation is shown schematically in figure 1 . It consists of a large cast-aluminum tank wound with heating coils

\footnotetext{
1 The research reported in this paper was supported in part by the ONR, and in part by contract AF 19 (122)-65 between the U. S. Air Force and the Ohio State University Research Foundation through sponsorship of the Geophysics Research Division of the U.S. Air Force Cambridge Research Center.

2 Present address, The Johns Hopkins University, Baltimore, Md.

Department of Physies, University of Oklahoma, Norman, Okla.

4 The Ohio State University, Columbus, Ohio.

5 Figures in brackets indicate the literature references at the end of this paper.
}

and covered with a layer of asbestos for insulation. One end is held in place by bolts and can be completely removed for adjusting the optical system. Three spherical mirrors, each of $100-\mathrm{cm}$ radius of curvature, are used in the optical system. Mirror $M_{3}$ is fixed, but an external control is provided for varying the inclination of the optic axes of mirrors $M_{1}$ and $M_{2}$. By means of this adjustment the path length of radiation passing through the cell can be changed in steps of $4 \mathrm{~m}$. Figure 1 shows the arrangement for a path length of $8 \mathrm{~m}$.

Rocksalt windows could not be used in this investigation, and it was found that silver-chloride windows reduced the energy very considerably. Satis. factory results were obtained by placing the Nernst filament, used as a source, inside the cell near mirror $M_{3}$ and leaving the exit window open. Radiation was reflected out of the cell and through this window by a small plane mirror.

Before introducing the steam, the cell was heated to $110^{\circ} \mathrm{C}$ to prevent condensation on the mirrors. The cell was filled with steam by displacement. When running spectra, a continuous stream of vapor from a gently boiling flask of distilled water entered at one end and escaped through the exit window.

For mapping regions of high absorption, air was allowed to enter the cell until a suitable concentration of water vapor was obtained. Near the center of the $\nu_{2}$ fundamental the atmospheric water vapor in the spectrometer alone was sufficient to produce very intense absorptions. The region from 5.7 to $6.7 \mu$ has been previously mapped by H. H. Nielsen [3] with very high resolution and with small amounts of water vapor. The present workers were unable to improve upon these results, and this region has not been remeasured. In the solar spectrum no energy is observed between 5.5 and $7.5 \mu$ at Columbus during most of the year.

The spectrometer with which these measurements were made has already been described [4]. For this work a 3,600 line/in replica echelette grating (width 5.5 in., height 3.5 in.) was used, together with a Perkin-Elmer thermocouple (rated sensitivity $8 \mathrm{v} / \mathrm{w}$ ) detector and their $13 \mathrm{c} / \mathrm{s}$ chopping system and amplifier. All spectra were recorded in the first order of the grating. Portions of the spectrum at short wavelengths were also scanned in the second 


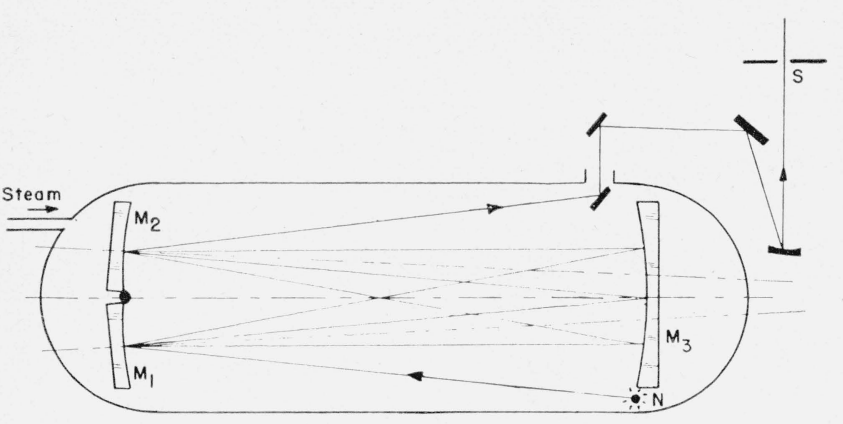

Figure 1. Multiple reflection cell.

order, but no improvement in the resolution was found.

After the spectrum of the 8-m path of water vapor had been obtained, the entire region was scanned by using the atmospheric path in the spectrometer alone. In this background spectrum, a line was found at $791 \mathrm{~cm}^{-1}$, which agrees in position with the Q-branch of a band of carbon dioxide. It is very weak in the laboratory spectrum and is probably caused by the carbon dioxide in the air path of the spectrometer.

To obtain the spectrum, small portions, corresponding to the rotation through $1 \mathrm{deg}$ of the grating circle, were scanned. The rocksalt foreprism was adjusted for maximum energy in the middle of each degree, which was then run at least twice in each direction. Fiduciary marks were made on the chart paper by a mechanical trigger device operated by an observer viewing coincidences of the grating circle markings with a graduated scale in the eyepiece of a microscope. The "central image" corresponding to the position of the grating for zero-order diffraction was measured during each day's work, in both directions. It was found to vary a few seconds of are from day to day.

Frequencies were calculated by using the formula

$$
\nu=n K / \sin \theta,
$$

where $n$ is the spectral order, $\nu$ the frequency, $K$ is a constant, and $\theta$ is the angular displacement from the central image. $K$ was determined from measurements of the 0.546073-, 0.576960-, and 0.579066- $\mu$ mercury lines in the orders from 10 to 16 using a low-pressure (H-2) mercury arc as a source and a 931A photomultiplier tube as detector. These measurements gave an average value for $K$ of $722.506 \pm 0.025 \mathrm{~cm}^{-1}$.

The four or more frequency measurements obtained for each line, including scannings in both directions, were averaged. These frequencies were then corrected to vacuum by assuming the laboratory air had an average refractive index of 1.0002565 , corresponding to a barometric pressure of about $740 \mathrm{~mm} \mathrm{Hg}$ and a temperature of $25^{\circ} \mathrm{C}$.

\section{Results}

A map of the water-vapor spectrum from 4.5 to $13 \mu$ is shown in figures 2 to 6 , with the exception of a short interval from 5.7 to $6.6 \mu$, where water-vapor absorption is very intense. To reach the longest wavelengths, the grating had to be used at extreme angles to the incident radiation, and very little energy was obtained even with the widest slits permissible. This accounts for the small deflection shown in figure 2 beyond $12 \mu$. As short portions of the spectrum were run individually, the total energy varied slightly, giving the segments shown in the figures. The approximate amounts of water vapor in the path for each region are given in the captions of the figures, as well as the temperature of the cell or spectrometer. It should be noted that when a temperature of $110^{\circ} \mathrm{C}$ is indicated, this refers to the temperature of the absorption cell. There was always some water vapor in the spectrograph at about $25^{\circ} \mathrm{C}$, also contributing to the absorption. This contribution is quite negligible when the cell contains steam at atmospheric pressure. The regions near the center of the $6.3-\mu$ band were run with only the air path in the spectrometer. Although spectra were recorded on a cold, dry day in February, many lines were still more intense than was desired.

The effective slit widths used varied from 0.22 $\mathrm{cm}^{-1}$ at $13 \mu$ to $0.83 \mathrm{~cm}^{-1}$ at $5 \mu$. These slit widths were approximately the same as those used in the investigation of the solar spectrum. Under these conditions the lines in the laboratory spectrum were wider than corresponding lines in the solar spectrum. Consequently, some lines clearly separated in the solar spectrum are blended in the laboratory spectrum. The increased line width observed in the spectrum of steam is caused both by the higher temperature and the much higher concentration of water vapor, giving a marked self pressure broadening effect. Approximately the same deflections were obtained in windows between absorption lines whether the cell was full of steam or air, indicating that there was little continuous absorption by water vapor in these windows. Thus the wings of watervapor lines lying outside the region investigated played an insignificant part in the absorption observed.

The lines have been numbered in order of increasing frequency in the figures as an aid to their identification in table 1. The line number is given in the first column of this table and the observed frequency, corrected to vacuum, in the second.

It is believed that the absolute values for the frequencies of well-defined lines are correct to better than $0.2 \mathrm{~cm}^{-1}$ at short wavelengths and to $0.1 \mathrm{~cm}^{-1}$ elsewhere. Most line frequencies have been given to $0.01 \mathrm{~cm}^{-1}$, but some of the very weak lines that are difficult to measure accurately are listed to the nearest $0.1 \mathrm{~cm}^{-1}$. A number of absorptions can be seen to consist of more than one line, and here the frequency of the position of the maximum absorption is listed to the nearest $0.1 \mathrm{~cm}^{-1}$. Scattered radiation did not amount to more than 10 percent of the total deflection anywhere in the region measured and would not give rise to any spurious lines.

When the solar spectrum is compared with the laboratory spectrum, it is found that the relative intensities of some of the lines are very different. 
This is to be expected because the amount of water vapor in the atmospheric path traversed by the solar radiation varies between 1,000 to $10,000 \mathrm{~atm}-\mathrm{cm}$ and is at an average temperature of about $14^{\circ} \mathrm{C}$, whereas the laboratory path was only $800 \mathrm{~atm}-\mathrm{cm}$ but at a temperature of $110^{\circ} \mathrm{C}$. The higher temperature of the laboratory sample gave intensities comparable with those in the solar spectrum to lines that originate from higher energy levels. However, lines arising from low-energy levels are little affected by the change in temperature from $14^{\circ}$ to $110^{\circ} \mathrm{C}$, and these lines were very weak or completely absent from the laboratory spectrum, although they may be quite prominent in the solar spectrum. This is illustrated by figures 7 and 8, which show small portions of the solar and laboratory spectra for comparison, together with the calculated spectra for the corresponding temperatures. The calculated spectra are schematically shown by drawing each line as a triangle, the width corresponding to geometric slit width used in the observed spectra, and the altitude proportional to the logarithm of the calculated intensity. The solar spectrum in figure 8 was observed during a cold winter day when the water-vapor content of the atmosphere was unusually low; so the calculated intensities for $14^{\circ} \mathrm{C}$ of table 1 were arbitrarily divided by 5 and plotted on the logarithmic scale indicated in the figure. Figure 7 also illustrates the greater line width of the laboratory steam spectrum than that of the solar spectrum.

In a number of cases where laboratory lines are very weak, or where several lines are blended, it is believed that more accurate frequencies can be obtained from the solar spectrum. Lines that appear in both spectra, but for which the frequencies from the solar spectrum have been used because they seem more reliable, are indicated by a dagger $(\dagger)$ in table 1. A few lines which appear only in the solar spectrum, but which can be fairly positively identified with water vapor because their intensities change with the amount of water vapor in the atmosphere or because they are theoretically predicted, have also been included in the table. Such lines have a dash in place of a line number.

The observed intensities of the lines are given in the next four columns. The first column gives the intensities of lines observed in the solar spectrum. A question mark in this column indicates that the intensity is uncertain because of absorption by other gases in the atmosphere. The values given are for "average" conditions of humidity and altitude of the sun. The intensity scale is very approximate, but runs from 0 to 100 , corresponding roughly to percentage absorption at the maximum. The next three columns give intensities as observed under various laboratory conditions; first, when water in the spectrometer alone was sufficient; next, when steam was diluted to a small fraction of its maximum value; and finally, when the full path of $800 \mathrm{~atm}-\mathrm{cm}$ was used. Figures 2 to 6 may be consulted for details. The steam was diluted over a wide range of concentrations so that the intensities for the diluted water vapor should be compared only for lines in the same neighborhood. In general, the absolute intensities of lines in the solar spectrum and the laboratory spectrum of the long path length of steam are roughly the same, although the intensities of the atmospheric lines vary considerably, depending on the altitude of the sun, the humidity, and the temperature of the atmosphere.

The sixth column of table 1 gives the identification of the line; or, if several transitions overlap to give an unresolved blend, the strongest components are listed. The identification consists of a letter symbol for the vibrational transition involved, the rotational quantum numbers of the upper state, $J_{\tau}^{\prime}$, and those of the lower state, $J_{\tau}^{\prime \prime}$. The letter symbol $a$ refers to pure-rotation transitions within the ground vibrational level. All identified lines with frequencies below $890 \mathrm{~cm}^{-1}$ are of this type, and they predominate up to $1,000 \mathrm{~cm}^{-1}$. Near $1,000 \mathrm{~cm}^{-1}$ is a region of minimum absorption by water vapor. At lower frequencies the pure-rotation lines become stronger, and at higher frequencies those of the $\nu_{2}$ band rapidly increase in intensity. The symbol $b$ identifies transitions in the $\nu_{2}$ fundamental. The remaining strong lines in the laboratory spectrum belong to this band. In addition, a few weak lines have been assigned to bands in which $\nu_{2}$ is the lower state: $c$ refers to the transition $2 \nu_{2}-\nu_{2}, d$ to $\nu_{1}-\nu_{2}$, and $e$ to $\nu_{3}-\nu_{2}$.

The seventh column gives the calculated frequencies derived from the term values of the corresponding rotational states. These are listed for the three lowest levels in table 2, which has been compiled partly from the present research, and partly from an extensive reinvestigation of the complete watervapor spectrum. The term values of the upper states of bands $d$ and $e$ have previously been given by Benedict and Plyler [5]. A calculated frequency in table 1 is the difference between two energy levels, $J_{\tau}^{\prime}$ and $J_{\tau}^{\prime \prime}$, involved in the transition. The calculated frequencies of a few lines have been placed in parentheses because in each case one of the levels concerned has been located by means of the observed line. These assignments depend on approximate predictions of the levels from extrapolations of regularities among the reliably known levels and on the agreement between calculated and observed intensities. For the remaining lines, however, other observed transitions connect the levels in question, and the agreement between observed and calculated frequencies is a true measure of the consistency of the measurements and the interpretation of the $\mathrm{H}_{2} \mathrm{O}$ spectrum.

The final columns of table 1 list relative values of the calculated intensities, for temperatures of $14^{\circ}$ and $110^{\circ} \mathrm{C}$. These temperatures approximately correspond to the average temperature in the solar and laboratory spectra, respectively. The tabulated numbers are, for $14^{\circ} \mathrm{C}, v(L S) g e^{-E^{\prime \prime} / k T} \cdot 10^{6}$, where $(L S)$ is the line strength, as defined and tabulated by Cross, Hainer, and King [6], $g$ is the statistical weight, and $E^{\prime \prime}$ is the energy of the lower state (from table 2 ). $v$ is a numerical factor for the intensity of the vibrational transitions relative to the pure-rotation band, which will be discussed presently. The factor $10^{6}$ was arbitrarily chosen so that medium-strength lines 
in the solar spectrum have intensities of the order of unity. For $110^{\circ} \mathrm{C}$ the factor was reduced to $10^{5}$ to compensate for the increased Boltzmann factor.

For the pure-rotation band, it is possible to calculate intensities on an absolute basis, since all quantities in the theoretical formula

$$
\int k_{\nu} d \nu=\frac{8 \pi^{3} N}{3 h c Q} p^{2} \nu \cdot(L S) g e^{-E^{\prime \prime} / k T}
$$

are known. $\int k_{\nu} d \nu$ is the integrated intensity of a line, $N, h, c$, and $k$ are universal constants, $Q$ is the state-sum, $\Sigma g e^{-E^{\prime \prime} / k T}$, and $p$ is the static dipolemoment, 1.86 Debye units. In order to convert our tabulated values for the pure-rotation band to the integrated intensity in units of $\mathrm{cm}^{-2} \mathrm{~atm}^{-1}$, one must multiply by $2.19 \times 10^{-7} \times \nu\left(\mathrm{cm}^{-1}\right)$, at $287^{\circ} \mathrm{K}$; at $383^{\circ} \mathrm{K}$ the factor is $1.07 \times 10^{-6} \times \nu\left(\mathrm{cm}^{-1}\right)$.

Not all of the transitions found in the course of this study are listed in the tables of reference [6]. The extension to $J \geq 12$ requires a moderate extrapolation. Transitions in the $R_{5,5}$ branch do not appear in their tables; according to a communication from Professor Cross, the line strength in nearly all these lines is less than 0.0001. For such lines the intensities have been estimated, and are placed in parentheses in table 1 ; the relative intensities at the two temperatures are significant, but the values may be in error by an order of magnitude.

The observed intensity of relatively weak lines in the pure rotation band, in both the solar and laboratory spectra, appear to be in fair agreement with the calculated absolute intensity. For example, the calculated intensity of line number one $\left(16_{-8}-15_{-10}\right)$ is $\int I_{\nu} d \nu=0.45 \times 2.19 \times 10^{-7} \times 760=7.3 \times$ $10^{-5} \mathrm{~cm}^{-2} \mathrm{~atm}^{-1}$. If the atmospheric path is $5 \times 10^{3}$ atm-cm, the equivalent width should be $0.36 \mathrm{~cm}^{-1}$, which is of the same order as given by the observed maximum absorption of 45 percent, assuming that the line has a half-width $0.10 \mathrm{~cm}^{-1}$ and is observed with a slit width of $0.22 \mathrm{~cm}^{-1}$. For the steam path of $800 \mathrm{~atm}-\mathrm{cm}$ the calculated equivalent width of the same line becomes $1.6 \times 1.07 \times 10^{-6} \times 760 \times 800=$ $1.04 \mathrm{~cm}^{-1}$, again in order of magnitude agreement with the observed maximum absorption of 68 percent for a line of half-width $0.4 \mathrm{~cm}^{-1}$.

For lines in $\nu_{2}$, and in the upper-state bands, the absolute value of the theoretical intensity cannot be calculated, as it depends on the empirical factor $v$. The following values of $v$ were chosen by which to multiply the pure-rotation intensities, in order to obtain fair over-all agreement with the observed intensities:

\begin{tabular}{|c|c|c|c|c|}
\hline Band_- & $b\left(\nu_{2}\right)$ & $c\left(2 \nu_{2}-\nu_{2}\right)$ & $d\left(\nu_{1}-\nu_{2}\right)$ & $e\left(\nu_{3}-\nu_{2}\right)$ \\
Factor_- & 0.005 & 0.01 & 0.0002 & 0.001 \\
\hline
\end{tabular}

These result in lines of the observed order of magnitude and are not unreasonable. The $\nu_{2}$ intensities, calculated in this way, appear to be somewhat weaker than the observed intensities, in the region 900 to $1,050 \mathrm{~cm}^{-1}$, and somewhat stronger than the observed intensities in the region 2,000 to $2,200 \mathrm{~cm}^{-1}$, by a factor of 2 or 3 in each case. These effects, which may be in part due to the greater effective slit width in the short-wave region, are similar to those encountered in other bands of $\mathrm{H}_{2} \mathrm{O}$, and even more strikingly in $\mathrm{H}_{2} \mathrm{~S}$ and other asymmetric-top molecules. An explanation of these effects has recently been proposed by Nielsen [7]. The anomaly in $\nu_{2}$ of $\mathrm{H}_{2} \mathrm{O}$ does not appear outstandingly large in the portions of the $P$ and $R$ branches near the origin, but is confined to the transitions of high $J$ and those in which $K$ changes by more than 1 .

\section{Discussion}

The agreement between the observed and calculated spectra as given in table 1, and illustrated in figures 7 and 8 , is quite complete, and argues well both for the accuracy of the measurements and the correctness of the interpretation. The lines not accounted for constitute less than 1 percent of the total absorption intensity. A few of the assignments to transitions of highest $J$, in both the purerotation spectrum and in $\nu_{2}$, are rather uncertain. It would be desirable to obtain further accurate measurements of steam in the region 550 to $770 \mathrm{~cm}^{-1}$ and of superheated steam or $\mathrm{H}_{2}-\mathrm{O}_{2}$ emission spectra throughout the rest of the vibration-rotation region, for confirmation. However, all the lower energy levels, up to $J=10$, are well accounted for, in that all predicted lines appear with approximately the correct intensity and frequency, and that no strong lines remain unidentified.

An additional demonstration that the spectral analysis is quite complete and essentially correct is afforded by the arrangement of the lines into series. Table 3 presents the lines of the four prominent $P$ branches of the $\nu_{2}$ vibration-rotation band, grouped in such a way as to display the regular decrease in frequency with increasing $J$. A similar array may be made for the lines in the $R$ branches, as well as for the Q branch lines, most of which, however, lie in the central region not measured in this study. Frequencies in parentheses are those of weak lines overlapped by a stronger component. It is clear, by a comparison of tables 3 and 1 , that the identification of many lines of higher $J$ cannot be expected without an increase of the path length, or, preferably, by increasing the temperature. As mentioned before, there is a distinct difference in temperature coefficient between the weaker lines of the $P_{1, \overline{1}}$, $P_{\overline{1}, \overline{1}}$, and $P_{\overline{1}, 1}$ branches, which have relatively high transition probabilities but originate from high-energy levels, and those of the $P_{\overline{3}_{1}}$ branch (as well as the few identified lines of the $P_{\overline{3}} .3$ branch, not included in table 3 ), which have lower line strengths but originate from lower energy levels. This difference manifests itself in the changed relative intensities in the laboratory and solar paths.

Table 4 is a similar presentation of the lines of the low-probability branches of the pure-rotation band. 
For completeness, there are included lower lines of the series, as observed by other investigators $[12,13]$ in the farther infrared, as well as frequencies, in parentheses, of lines that have not yet been measured with high accuracy. A comparison with table 1 again shows that the suggested array accounts for most of the observed lines from 760 to $1,000 \mathrm{~cm}^{-1}$, with the lines that are most enhanced in the atmosphere belonging, for the most part, to the more highly forbidden $R_{5, \overline{3}}$ and $R_{5, \overline{5}}$ series.

The energy levels of $\nu_{2}$, which are now known with completeness and good precision up to $J=9$, permit an improved determination of the rotational constants for that vibrational level. The method is described elsewhere. Figure 9 presents the effective rotational constant $A_{J}^{\prime}, \mathrm{B}_{J}^{\prime \prime}$, and $\mathrm{C}_{J}^{\prime}$ for each $J$, derived from the levels of table 2, plotted against $J(J+1)$. The points are seen to fall nicely on smooth curves. The intercepts give the rotational constants $A, B$, and $C$, and the limiting slopes give the centrifugal stretching constants $D_{A}, D_{B}$, and $D_{C}$. These are listed in table 5 and compared with the corresponding constants of the ground state. There is an appreciable curvature in the plot of $A_{J}^{\prime}$, showing that centrifugal stretching terms of higher order are important at relatively low $J$.

The few weak lines that are not assigned may be transitions of higher $J$ in $\nu_{2}$, upper-state transitions, or lines in the isotopic molecules $\mathrm{H}_{2} \mathrm{O}^{18}$ or $\mathrm{HDO}$. The calculated vibrational shift for $\nu_{2}$ of $\mathrm{H}_{2} \mathrm{O}^{18}$ is $-6.5 \mathrm{~cm}^{-1}$; this shift would be increased for lines in the $R$ branch and diminished in the $P$ branch. Tentative assignments of some of the observed lines can be made on this basis; for example, line 285 $\left(1414.99 \mathrm{~cm}^{-1}\right)$, which lies $4.3 \mathrm{~cm}^{-1}$ below the strong line $3_{3}-4_{3}$, and line $353\left(1764.22 \mathrm{~cm}^{-1}\right)$, which lies $8.4 \mathrm{~cm}^{-1}$ below the strong line $3_{3}-2_{1}$, have the proper intensity ratio $(1: 500)$ and positions to be their analogues in $\mathrm{H}_{2} \mathrm{O}^{18}$. Other such possible lines are suggested in the table. It should be noted that, on the basis of the expected relative intensities, it would be very unlikely that many absorption lines due to HDO would appear. Although absorption due to the $\nu_{2}$ band of this isotope has been postulated by Adel [8] in the $7.2 \mu$ region of the solar spectrum, and the $\nu_{1}$ fundamental has been definitely observed, resolved, and analyzed in the solar spectrum at $3.7 \mu[9,10]$, the intensity of the lines of the $\nu_{2}$ fundamental of $\mathrm{HDO}$ relative to those of $\mathrm{H}_{2} \mathrm{O}$ on our spectra would be very small. The frequencies of the $\nu_{2}$ fundamental of HDO published by Barker and Sleator [11] do not show any definite coincidences with our data. There should, however, be a number of fairly strong lines at lower frequen- cies than their work reached, which would lie in the region of weaker $\mathrm{H}_{2} \mathrm{O}$ absorption and might thus appear as weak lines in our spectra. For example, on the basis of the known structure of $\mathrm{HDO}$, we calculate that the line $3_{3}-4_{3}$ should fall near $1,250 \mathrm{~cm}^{-1}$. Line 183 , otherwise unaccounted for, has about the proper intensity in both laboratory and solar spectra for this transition.

It may also be of interest to point out that the completeness of the assignment, and the fact that the intensity differences in the spectra between atmospheric water vapor and nearly saturated steam can be explained in detail on the basis of temperature, is strong evidence, although of a negative sort, against the existence of $\left(\mathrm{H}_{2} \mathrm{O}\right)_{2}$ molecules in the saturated vapor. The association of $\mathrm{H}_{2} \mathrm{O}$ in the liquid phase is abundantly proved by the marked lowering of frequency in the $\nu_{3}$ fundamental and a somewhat smaller shift in $\nu_{2}$. The existence of dimers in the vapor has often been postulated to account for changes in density, dielectric polarization, etc., near saturation. If such dimers exist as molecules with quantized vibrationrotation states, the evidence of the present study would be that their concentration, relative to $\mathrm{H}_{2} \mathrm{O}$ monomer, must be less than 1 percent, since in the region of $1,400 \mathrm{~cm}^{-1}$, where $\left(\mathrm{H}_{2} \mathrm{O}\right)_{2}$ should be most strongly absorbing, the unaccounted-for absorption is very small. We have mentioned previously that the steam spectrum shows a somewhat greater line width than the atmospheric spectrum, but there is no appreciable frequency shift, and the amount of self-broadening is by no means abnormally large for a polar molecule.

\section{References}

[1] A. Adel, Astrophys. J. 94, 451 (1941).

[2] J. U. White, J. Opt. Soc. Am. 32, 285 (1942).

[3] H. H. Nielsen, Phys. Rev. 59, 565 (1941).

[4] J. H. Shaw, R. M. Chapman, J. N. Howard, and M. L. Oxholm, Astrophys. J. 113, 268 (1951).

[5] W. S. Benedict and E. K. Plyler, J. Research NBS 46, 246 (1951) RP2194.

[6] P. C. Cross, R. M. Hainer, and G. W. King, J. Chem. Phys. 12, 210 (1944).

[7] H. H. Nielsen, Phys. Rev. 83, 838 (1951).

[8] A. Adel, Astrophys. J. 93, 509 (1941).

[9] R. M. Chapman and J. H. Shaw, Phys Rev. 78, 71 (1950).

[10] W. Benesch, J. Strong, and W. S. Benedict, Progress Report to ONR, Contract N50ri-166 (Aug. 1, 1950).

[11] E. F. Barker and W. W. Sleator, J. Chem. Phys. 3, 660 (1935).

[12] H. M. Randall, D. M. Dennison, N. Ginsburg, and L. R. Weber, Phys. Rev. 52, 160 (1937).

[13] L. R. Weber and H. M. Randall, Phys. Rev. 40, 835 (1932); C. S. Rupert, J. H. Taylor, and J. Strong, private communication. 


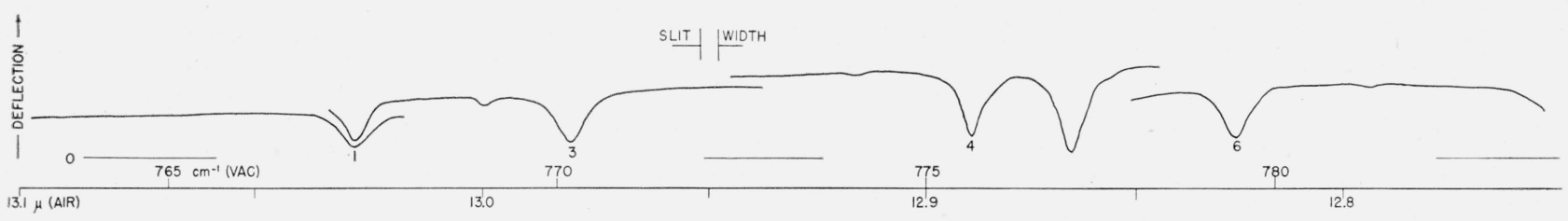

$-1 \vdash$
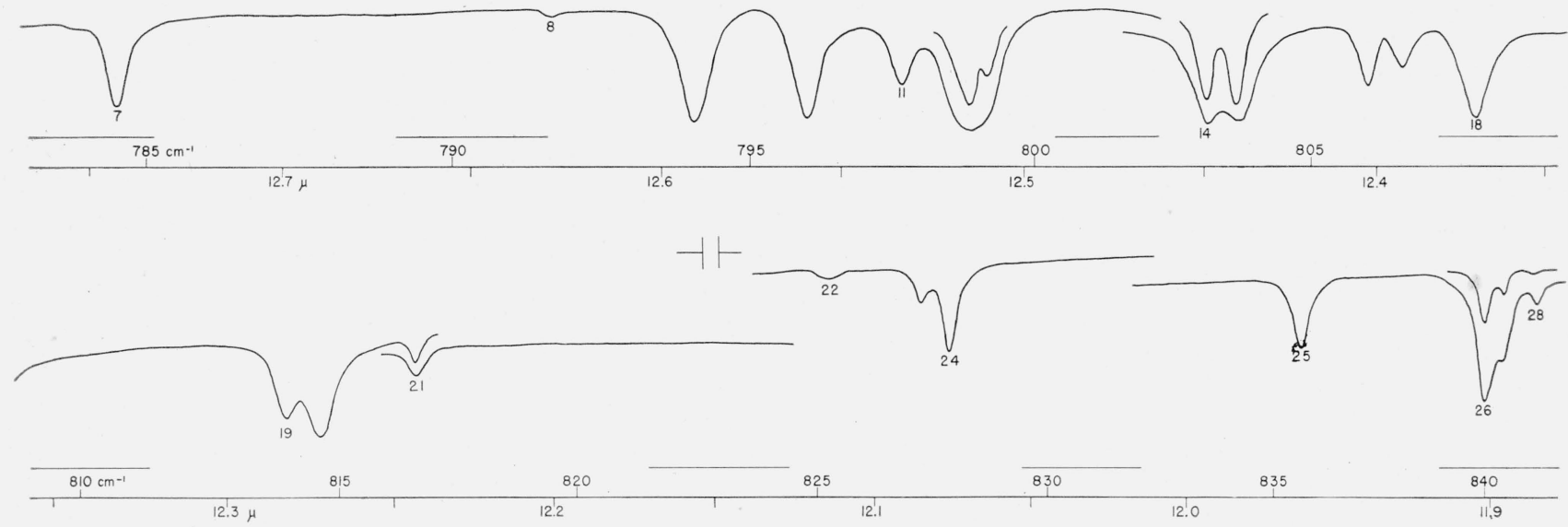

FIGURE 2. Absorption spectrum from 11.9 to $13.1 \mu$ of an eight meter path of water vapor at $110^{\circ} \mathrm{C}$ and one atmosphere pressure.
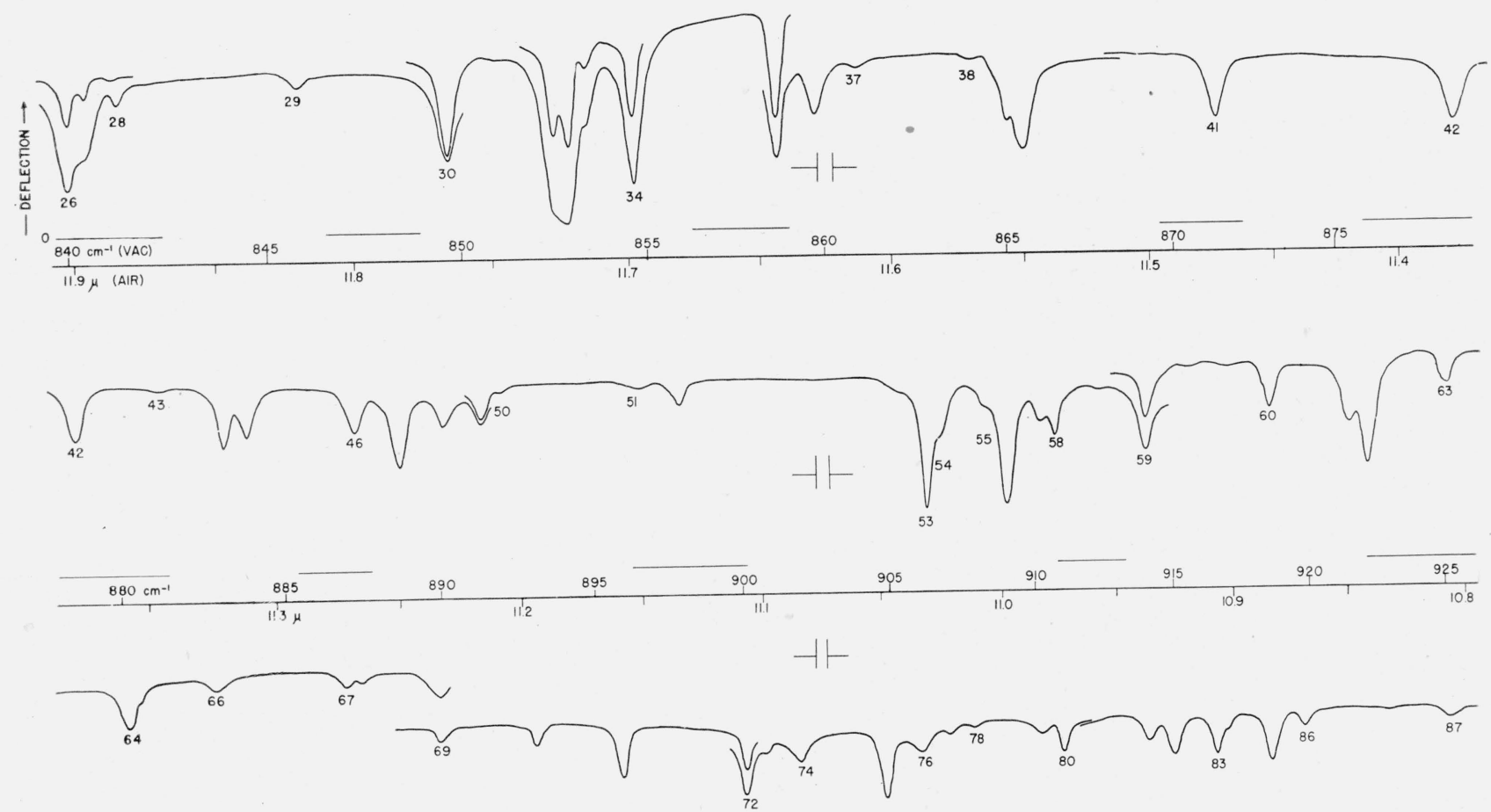

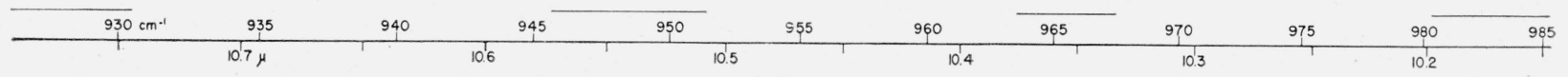

FIGURE 3. Absorption spectrum from 10.2 to $11.9 \mu$ of an eight meter path of water vapor at $110^{\circ} \mathrm{C}$ and one atmosphere pressure. 

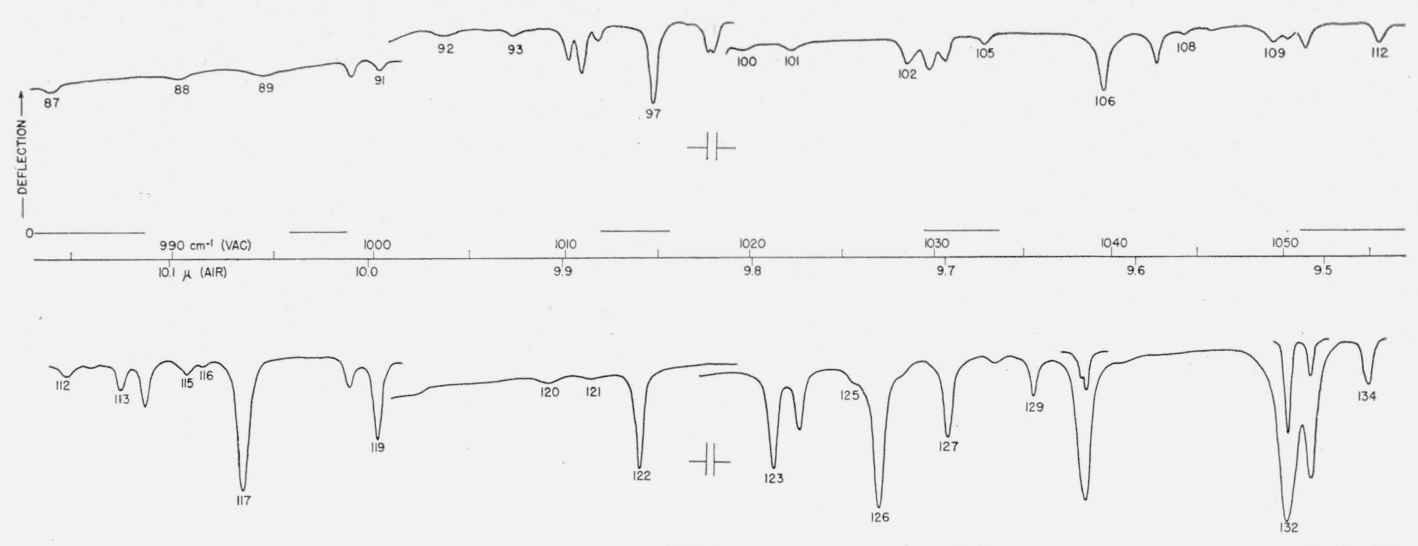

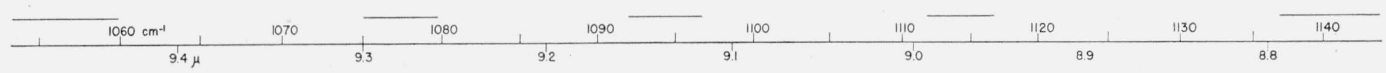

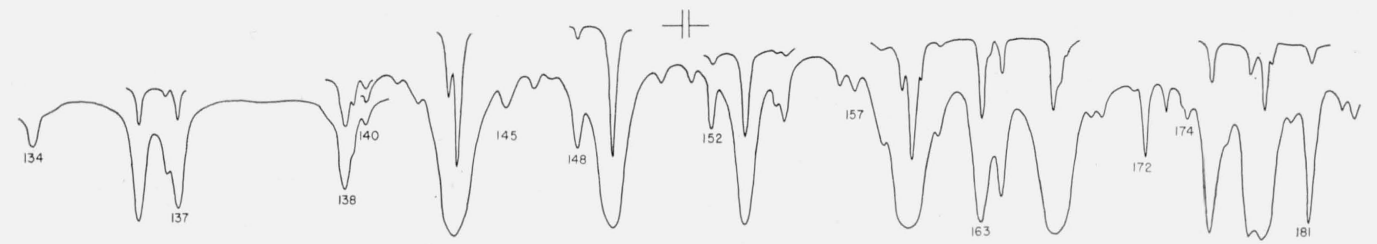

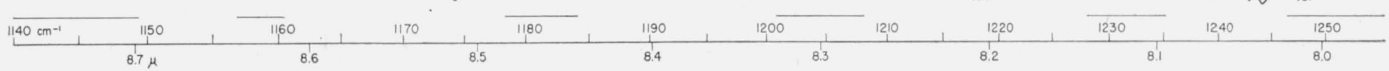

FIGURE 4. Absorption spectrum from 10.2 to $11.9 \mu$ of an eight meter path of water vapor at $110^{\circ} \mathrm{C}$ and one atmosphere pressure and with small sections also at lower concentration.
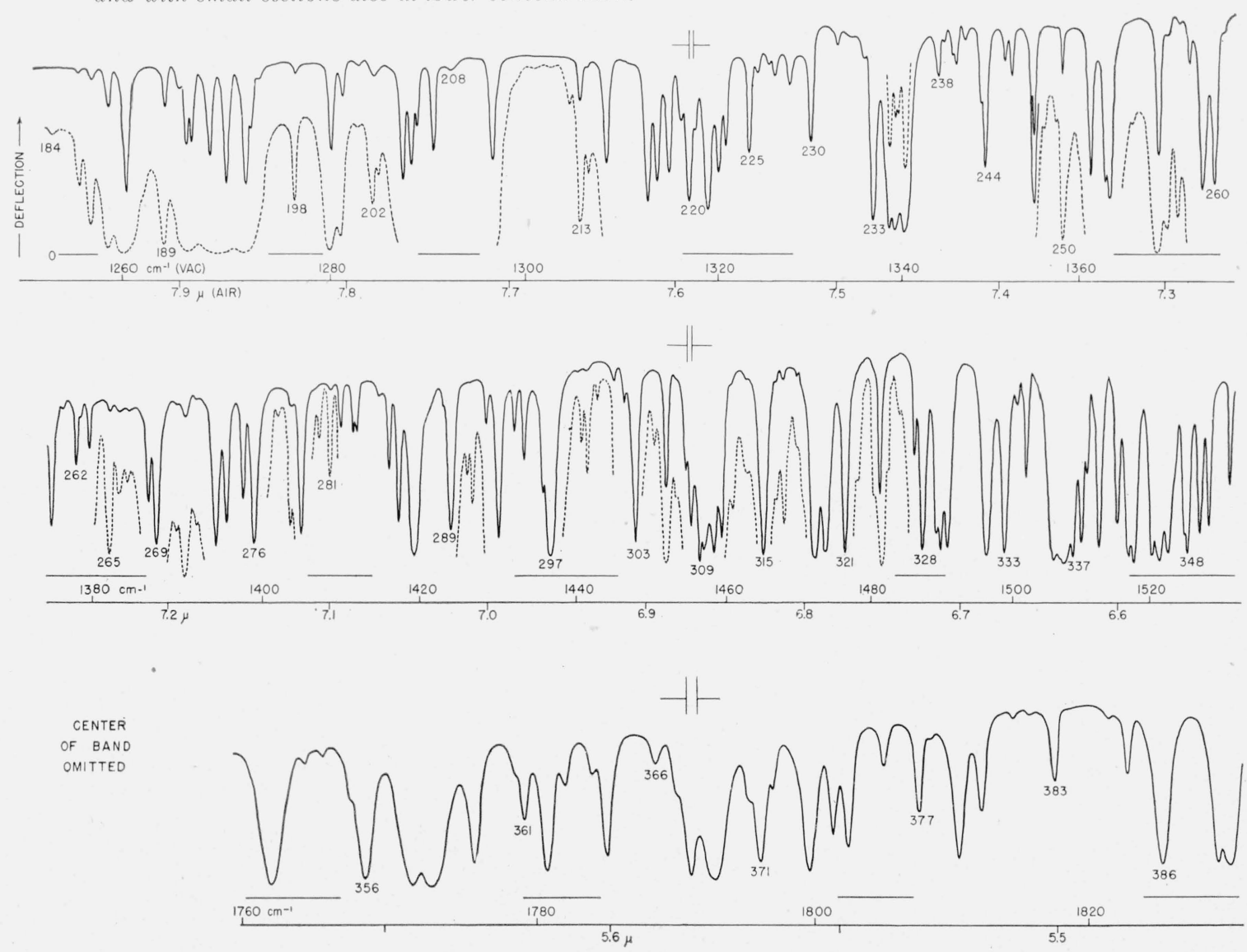

Figure 5. Absorption spectrum from 5.5 to $5.7 \mu$ and from 6.6 to $8.0 \mu$ of water vapor.

Solid line refers to water vapor in the air of the spectrometer at approximately $25^{\circ} \mathrm{C}$, dotted line to an eight meter path at $110^{\circ} \mathrm{C}$ and various concentrations of water vapor in air. 

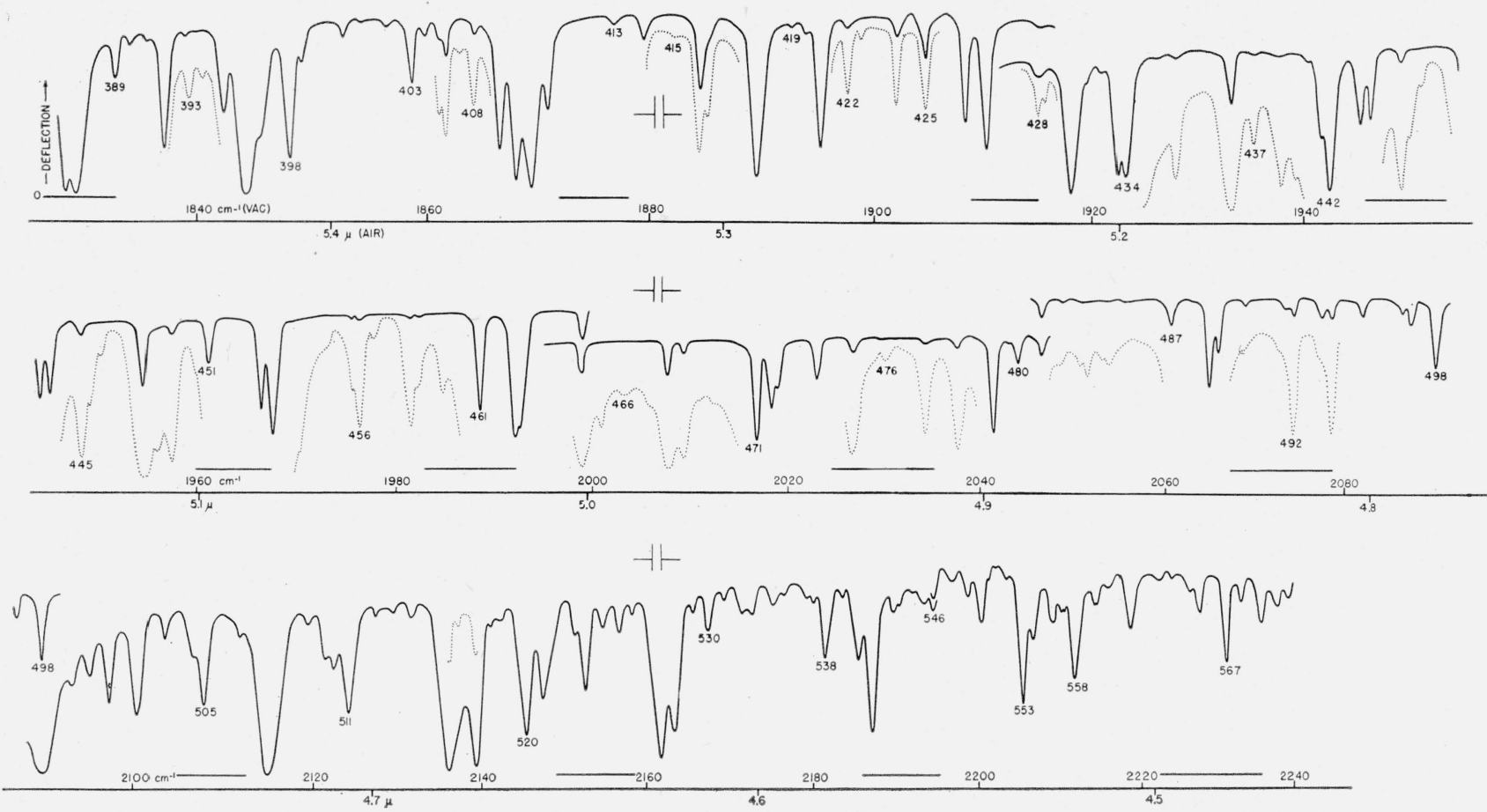

Figure 6. Absorption spectrum from 4.5 to $5.5 \mu$ of water vapor.

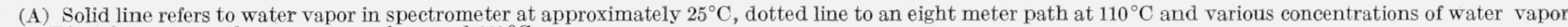
in air; (B) eight meter path at one atmosphere and $110^{\circ} \mathrm{C}$,
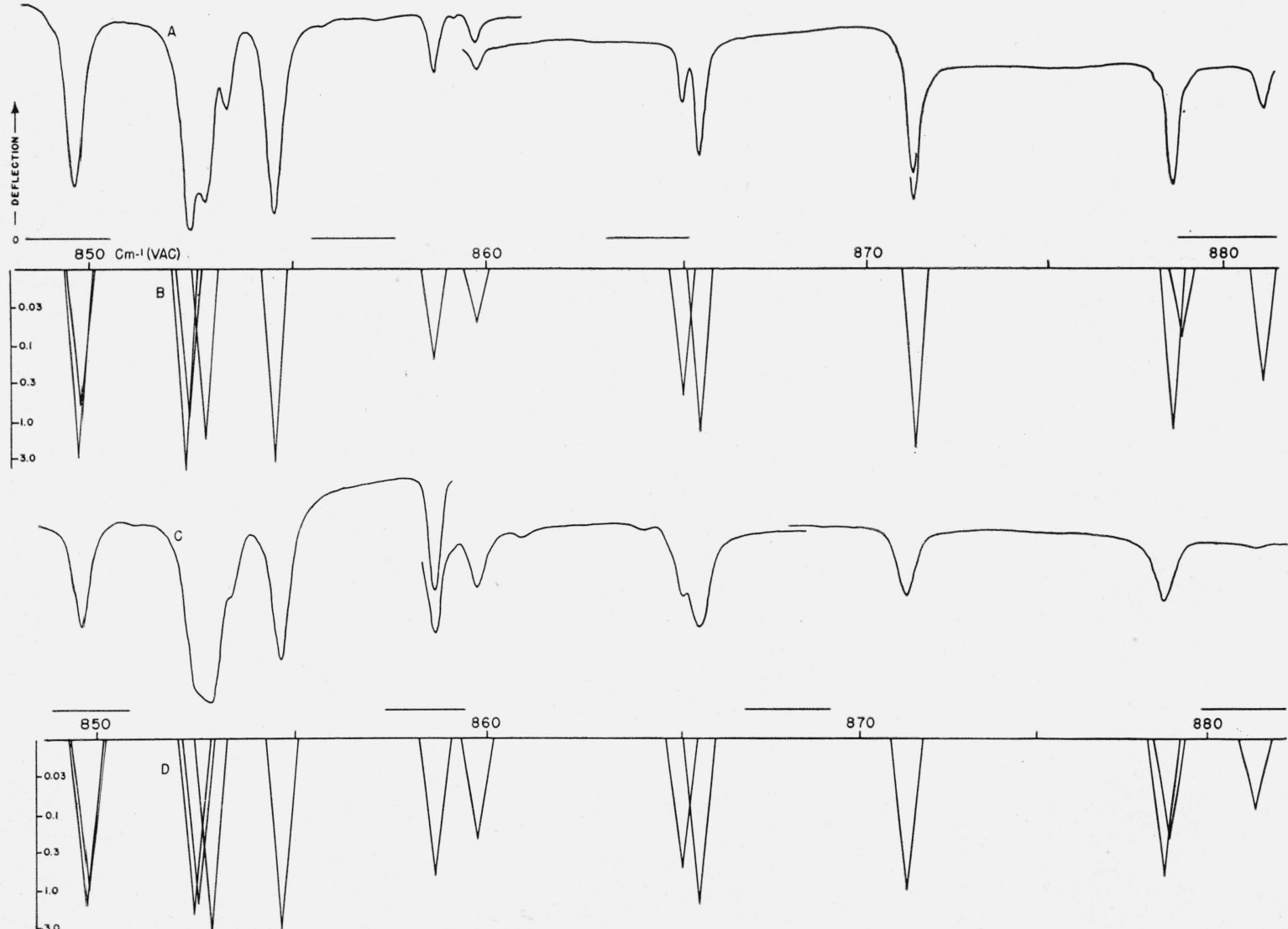

FiguRE 7. Solar, laboratory, and calculated spectra of the region from 850 to $880 \mathrm{~cm}^{-1}$.

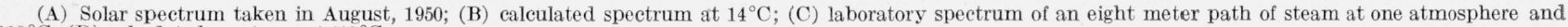
$110^{\circ} \mathrm{C}$; (D) calculated spectrum at $110^{\circ} \mathrm{C}$. 

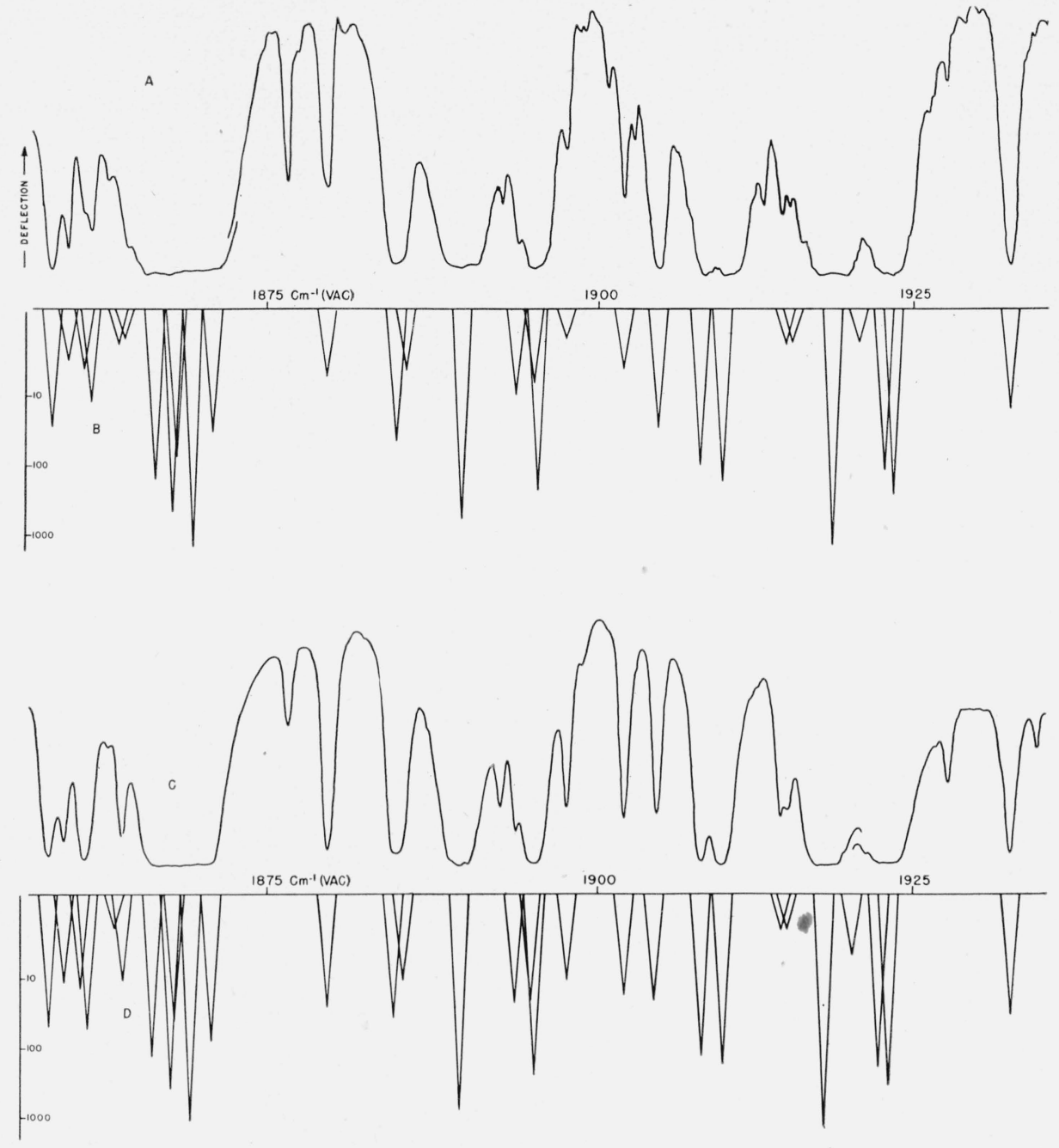

Figure 8. Solar, laboratory and calculated spectra of the region from 1860 to $1925 \mathrm{~cm}^{-1}$.

(A) Solar spectrum taken in February 1951 , with an air temperature of $14^{\circ} \mathrm{C}$, (B) at 14 , (C) laboratory spectrum $110^{\circ} \mathrm{and}$ (D) calculated spectrum at $110^{\circ} \mathrm{C}$.

Figure 9. Curves from which may be derived the inertial and centrifugal stretching constants associated with rotation about the three axes of inertia: $(A)$ The least axis; (B) the intermediate axis; (C) the greater axis.

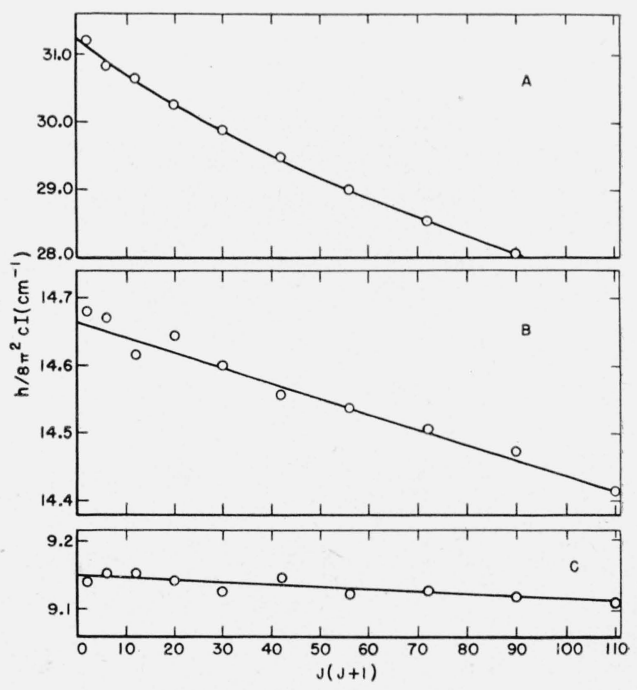


TABLE 1. Absorption Spectrum of $\mathrm{H}_{2} \mathrm{O}$ between $4.5 \mu$ and $13 \mu$.

\begin{tabular}{|c|c|c|c|c|c|c|c|c|}
\hline \multirow{4}{*}{ 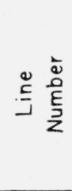 } & \multicolumn{4}{|c|}{ OBSERVED DATA } & \multirow{4}{*}{$\begin{array}{l}\text { Identification } \\
J_{\tau}^{\prime}-J_{\tau}^{\prime \prime}\end{array}$} & \multicolumn{3}{|c|}{ CALCULATED DATA } \\
\hline & \multirow{3}{*}{$\begin{array}{c}\text { Wave } \\
\text { Number } \\
\mathrm{cm}^{-1} \text { (vac) }\end{array}$} & \multicolumn{3}{|c|}{ Intensity } & & \multirow{3}{*}{$\begin{array}{c}\text { Wave } \\
\text { Number } \\
\mathrm{cm}^{-1} \text { (vac) }\end{array}$} & \multicolumn{2}{|c|}{ Intensity } \\
\hline & & \multirow{2}{*}{ Solar } & & aboratory & & & & \\
\hline & & & $\begin{array}{l}\text { Air } \\
\text { only }\end{array}$ & 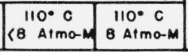 & & & $14^{\circ} \mathrm{C}$ & $110 . \mathrm{c}$ \\
\hline
\end{tabular}

$\begin{array}{llllllllllll}1 & 767.37 \quad 45 \quad & -68 & 2 & 16 & -8 & 15.10 & (67.4) & 0.44 & 1.6\end{array}$

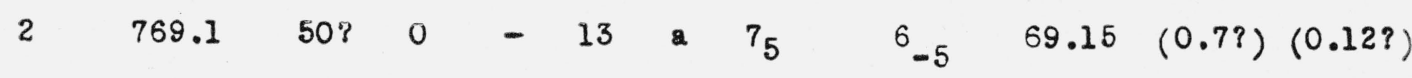

$\begin{array}{llllllllllll}3 & 770.17 \quad 60 ? \quad 0 \quad 12_{2} & 11 & 4 & 70.17 & 3.6 & 3.5\end{array}$

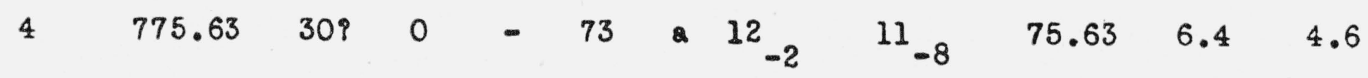

$\begin{array}{lllllllllllll}5 & 777.07 & 60 ? & 0 & - & 94 & 10_{5} & 9_{1} & 77.09 & 11 & 7.5\end{array}$

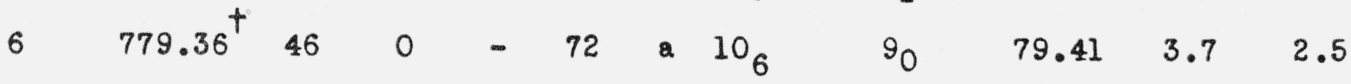

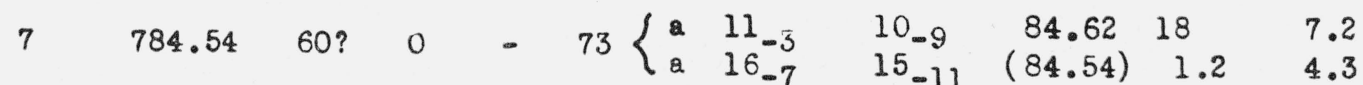
$8^{8} \quad 791.6 \quad-5-5$

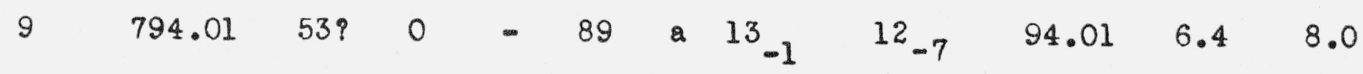

$\begin{array}{llllllllllll}10 & 796.01 & 85 & 0 & - & 88 & * & 10_{1} & 9_{-7} & 96.08 & 20 & 8.1\end{array}$

$\begin{array}{llllllllllll}11 & 797.65 & 50 & 0 & - & 56 & \text { a } & 11_{4} & 10_{0} & 97.66 & 2.3 & 1.9\end{array}$

$\begin{array}{lllllllllllll}12 & 798.75 & 99 & 0 & 73 & 95 & \text { a } & 15 & -11 & 14-13 & (98.75) & 6.8 & 9.0\end{array}$

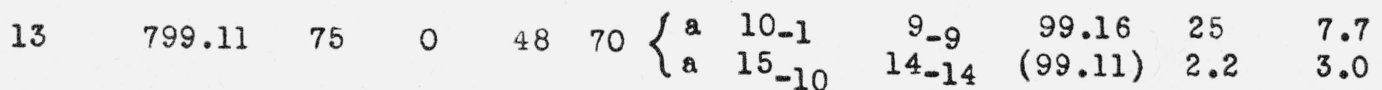

$\begin{array}{llllllllllll}14 & 803.09^{\dagger} & 85 & 0 & - & 88 & 8 & 11_{5} & 10_{-1} & 03.10 & 6.7 & 5.5\end{array}$

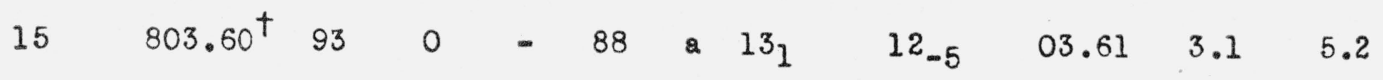

$\begin{array}{lllllllllllll}16 & 806.06 \quad 30 \quad 0 \quad & - & 54 & 2 & 16 & -10 & 15 & -12 & (06.06) & 0.3 & 0.75\end{array}$

$17 \quad 806.75 \quad 10 \quad 0 \quad-\quad 36$

$18\left\{\begin{array}{l}808.14^{\dagger}+80 \\ 808.33^{\dagger}\end{array}\right\} \quad-\quad 80\left\{\begin{array}{cccccc}a & 16-9 & 15-13 & (08.14) & 0.9 & 2.2 \\ a & 85 & 7_{-3}^{-3} & 08.29 & 1.8 & 0.53\end{array}\right.$

$\begin{array}{llllllllllllll}19 & 813.97 & 92 & 0 & - & 62 & \text { a } & 9_{9} & 8_{3} & 13.97 & 2.5 & 2.2\end{array}$

$\begin{array}{llllllllllll}20 & 814.61 & 95 & 0 & - & 77 & \text { a } & 12_{3} & 11_{-1} & 14.60 & 3.4 & 4.1\end{array}$

See footnotes at ond of table. 
TABLE 1-Continued.

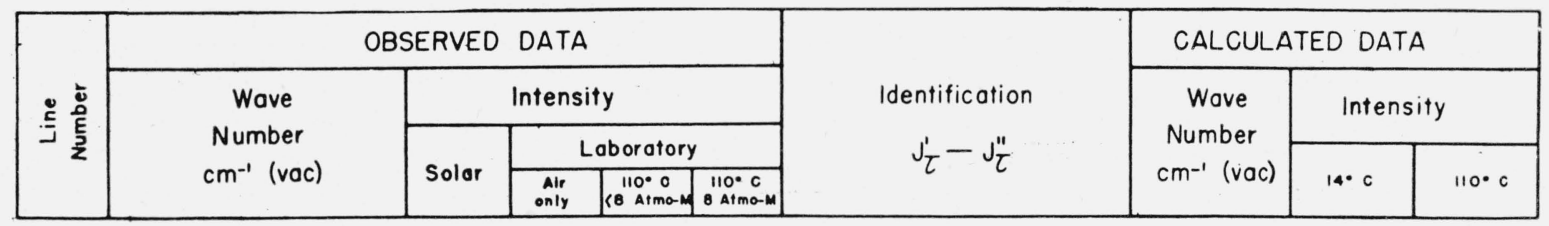

$21 \quad 816.55 \quad 10 \quad 0 \quad-\quad 20$

$22825.24^{\dagger} \quad 85 \quad 0 \quad-\quad 5 \quad \begin{array}{llllllll}8 & 5 & 7_{4} & 25.16 & 1.0 & 0.4 ?\end{array}$

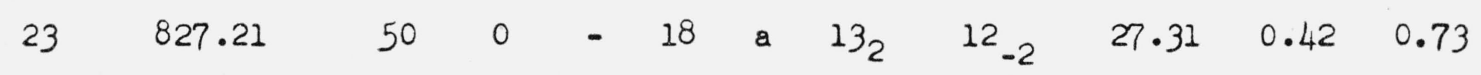

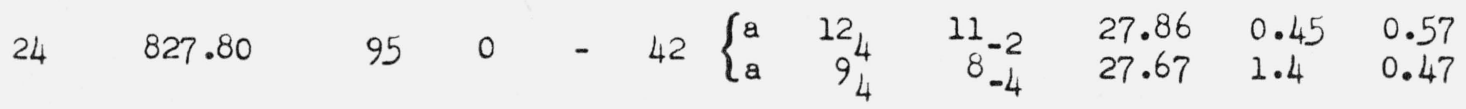

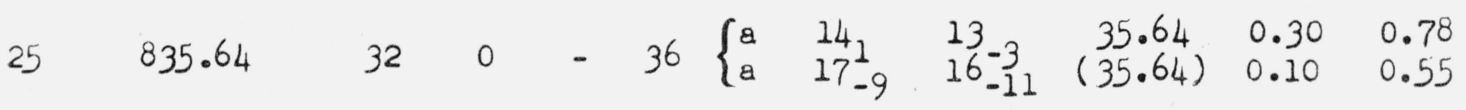
$26 \quad 840.01 \quad 90 \quad-66\left\{\begin{array}{llllll}a & 10 & 9 & 40.01 & 1.6 & 1.7 \\ a & 10 & 9 & 40.15 & 0.53 & 0.56\end{array}\right.$ $27 \quad 840.36^{\dagger} \quad 45 \quad 0 \quad-\quad \begin{array}{lllllllll}46 & \text { a } & 14 & 14 & 13.6 & (40.36) & 0.24 & 0.50\end{array}$

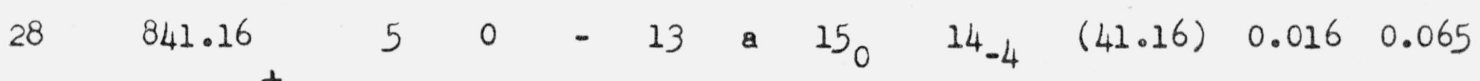

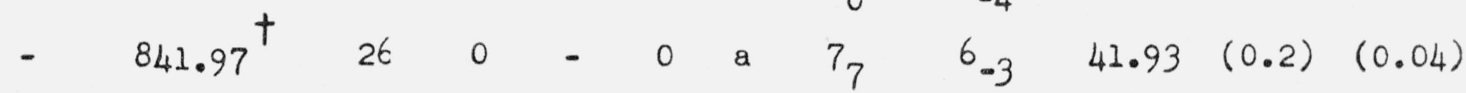
$29 \quad 845.83 \quad 3 \quad 0 \quad-9$

$\begin{array}{lllllllllll}30 & 849.69 & 78 & 0 & 56 & \text { a } & 110 & 10.8 & 49.79 & 2.9 & 1.6\end{array}$

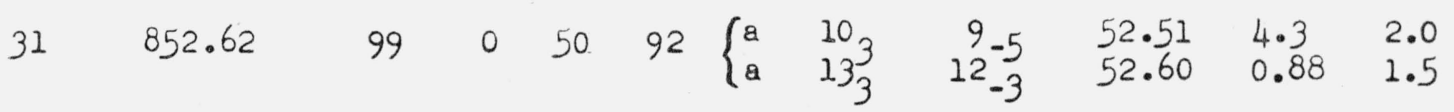

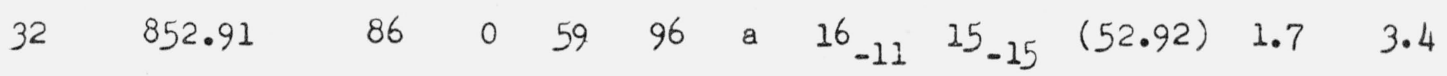
$\begin{array}{llllllllllll}33 & 853.41 & 40 & 0 & 12 & 40 & \text { a } & 14.2 & 13.8 & 53.41 & 0.59 & 1.0\end{array}$

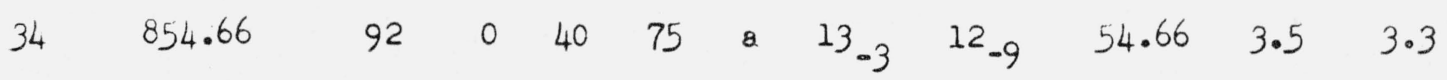
$\begin{array}{lllllllllllll}35 & 858.65 & 27 & 0 & - & 49 & a & 17_{-11} & 16_{-13} & (58.65) & 0.15 & 0.64\end{array}$ $\begin{array}{lllllllllllll}36 & 859.78 & 9 & 0 & - & 29 & \text { a } & 17 & -10^{16} & 14 & (59.78) & 0.05 & 0.21\end{array}$ $37 \quad 861.0 \quad-0 \quad-4$ $38 \quad 864 \cdot 0 \quad-0 \quad-\quad 3$ $\begin{array}{llllllllllll}39 & 865.02^{\dagger} \quad 32 & 0 & - & 37 & \text { a } & 116 & 11_{2} & 65.01 & 0.46 & 0.49\end{array}$

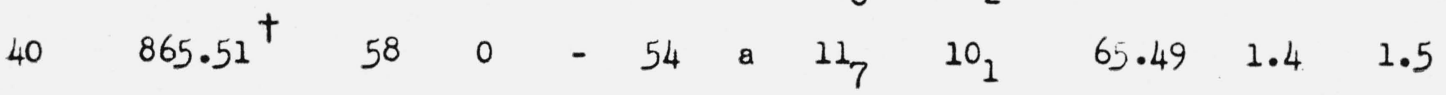


TABLE I-Continued.

\begin{tabular}{|c|c|c|c|c|c|c|c|c|c|c|c|}
\hline \multirow{4}{*}{ 导总 } & \multicolumn{5}{|c|}{ OBSERVED DATA } & & & & \multicolumn{3}{|c|}{ CALCULATED DATA } \\
\hline & \multirow{3}{*}{$\begin{array}{c}\text { Wave } \\
\text { Number } \\
\mathrm{cm}^{-1} \text { (vac) }\end{array}$} & \multicolumn{4}{|c|}{ Intensity } & \multirow{3}{*}{\multicolumn{3}{|c|}{$\begin{array}{c}\text { Identification } \\
J_{\tau}^{\prime}-J_{\tau}^{\prime \prime}\end{array}$}} & \multirow{3}{*}{$\begin{array}{c}\text { Wave } \\
\text { Number } \\
\mathrm{cm}^{-1} \text { (vac) }\end{array}$} & \multicolumn{2}{|c|}{ Intensity } \\
\hline & & \multirow{2}{*}{ Solar } & \multicolumn{3}{|c|}{ Laboratory } & & & & & & \\
\hline & & & & & & & & & & & \\
\hline 41 & $871.32^{\dagger}$ & 78 & 0 & - & 36 & $a$ & $11-2$ & $10-10$ & 71.49 & 2.3 & 0.92 \\
\hline 42 & $878.61^{\dagger}$ & 68 & 0 & - & 36 & $a$ & $\begin{array}{l}12_{-4} \\
14_{2}\end{array}$ & $\begin{array}{l}11-10 \\
13-4\end{array}$ & $\begin{array}{l}78.70 \\
78.9\end{array}$ & $\begin{array}{l}1.3 \\
0.08\end{array}$ & $\begin{array}{l}0.65 \\
0.20\end{array}$ \\
\hline 43 & $881.15^{\dagger}$ & 23 & 0 & - & 2 & 2 & $8_{6}$ & $7-4$ & 81.20 & $(0.3)$ & $(0.08)$ \\
\hline 44 & 883.18 & 24 & 0 & - & 32 & $a$ & $15-1$ & $14-7$ & $(83.18)$ & 0.13 & 0.18 \\
\hline 45 & 883.89 & 59 & 0 & - & 25 & $a$ & $11_{2}$ & $10_{-6}$ & 84.01 & 0.95 & 0.57 \\
\hline 46 & 887.33 & 72 & 0 & - & 22 & $\mathbf{a}$ & $9_{3}$ & $8-7$ & 87.44 & $(1.1)$ & $(0.27)$ \\
\hline 47 & 888.71 & 46 & 0 & - & 41 & $a$ & $12_{5}$ & ${ }^{11} 1$ & 88.71 & 0.63 & 0.90 \\
\hline 48 & 890.14 & 26 & 0 & - & 16 & $\mathbf{a}$ & $12_{6}$ & $11_{0}$ & 90.16 & 0.21 & 0.30 \\
\hline 49 & 891.33 & 41 & 0 & - & 17 & $a$ & $10_{9}$ & $9_{5}$ & 91.33 & 0.28 & 0.26 \\
\hline 50 & $892.1^{\dagger}$ & 6 & 0 & - & 4 & & & & & & \\
\hline 51 & $896.57^{\dagger}$ & 10 & 0 & - & 4 & $b$ & $12-12$ & $13-8$ & 96.53 & 0.006 & 0.009 \\
\hline 52 & $897.77^{\dagger}$ & 22 & 0 & - & 14 & $b$ & $12-11$ & $13-9$ & 97.77 & 0.017 & 0.027 \\
\hline - & $905.50^{\dagger}$ & 10 & 0 & - & 0 & $\mathrm{~b}$ & $10_{-7}$ & $11-1$ & 05.34 & 0.012 & 0.014 \\
\hline 53 & 906.32 & 56 & 0 & - & 69 & $a$ & ${ }^{17}-13$ & $16-15$ & $(05.32)$ & 0.3 & 0.8 \\
\hline 54 & 906.8 & 13 & 0 & - & 30 & $a$ & $15_{1}$ & $14-5$ & $(06.6)$ & 0.06 & 0.09 \\
\hline 55 & $908.09^{t}$ & 15 & 0 & - & 12 & & & & & & \\
\hline 56 & 909.02 & 83 & 0 & - & 67 & a & $12-1$ & $11_{-9}$ & 09.12 & 3.0 & 2.0 \\
\hline 57 & 910.17 & 20 & 0 & - & 21 & $\mathbf{a}$ & $13_{4}$ & $12_{0}$ & 10.4 & 0.08 & 0.17 \\
\hline 58 & 910.77 & 12 & 0 & - & 27 & $a$ & ${ }^{18}-11$ & ${ }^{17}-15$ & $(10.8)$ & 0.02 & 0.12 \\
\hline 59 & 914.06 & 34 & 0 & - & 33 & $a$ & $13_{5}$ & $12-1$ & 14.06 & 0.24 & 0.50 \\
\hline 60 & 918.52 & 37 & 0 & - & 23 & $\mathbf{a}$ & $1^{1} \mathrm{~g}$ & $10_{3}$ & 18.52 & 0.25 & 0.31 \\
\hline
\end{tabular}


TABLE 1-Continued.

\begin{tabular}{|c|c|c|c|c|c|c|c|c|}
\hline \multirow{4}{*}{ 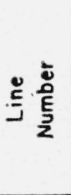 } & \multicolumn{4}{|c|}{ OBSERVED DATA } & \multirow{4}{*}{$\begin{array}{l}\text { Identification } \\
J_{\tau}^{\prime}-J_{\tau}^{\prime \prime}\end{array}$} & \multicolumn{3}{|c|}{ CALCULATED DATA } \\
\hline & \multirow{3}{*}{$\begin{array}{c}\text { Wave } \\
\text { Number } \\
\mathrm{cm}^{-1} \text { (vac) }\end{array}$} & \multicolumn{3}{|c|}{ Intensity } & & \multirow{3}{*}{$\begin{array}{c}\text { Wave } \\
\text { Number } \\
\mathrm{cm}^{-1} \text { (voc) }\end{array}$} & \multicolumn{2}{|c|}{ Intensity } \\
\hline & & \multirow{2}{*}{ Solar } & & bor.otory & & & & \\
\hline & & & $\begin{array}{l}\text { Alr } \\
\text { only }\end{array}$ & \begin{tabular}{|c|c|}
$110^{\circ} 0$ & $110^{\circ}-\mathrm{C}$ \\
$8^{\circ}$ Atmo-n & o Atmo-m \\
\end{tabular} & & & $14^{\circ} \mathrm{C}$ & $1100 \mathrm{c}$ \\
\hline
\end{tabular}

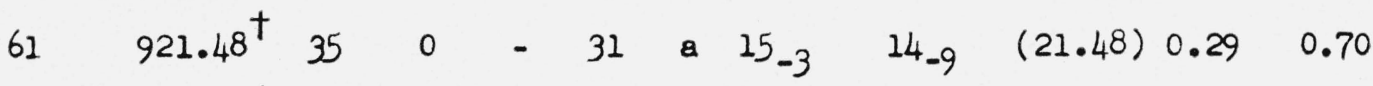
$\begin{array}{llllllllllll}62 & 922.19^{\dagger} 89 & 0 & - & 52 & \text { a } & 12 & 11 & 11 & 22.41 & 1.5 & 1.2\end{array}$ $63925.05^{\dagger} 80 \quad 0 \quad-\quad \begin{array}{lllllll}15 & \text { a } & 9 & 9_{5} & 25.12 & (1.0) & (0.3)\end{array}$ $\begin{array}{lllllllllllll}64 & 929.00 & 22 & 0 & - & 18 & 18 & 14 & 13 & & 29.00 & 0.063 & 0.20\end{array}$ $\begin{array}{llllll}65 & 929.4 & 0 & 0 & -\end{array}$

$\begin{array}{llllll}66 & 932.32 & 1 & 0 & - & 5\end{array}$

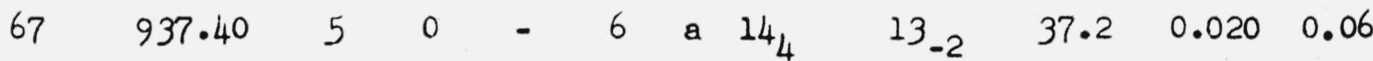
$\begin{array}{llllll}68 & 938.2 & 2 & 0 & - & 4\end{array}$

$\begin{array}{llllllllllll}69 & 941.12 & 20 & 0 & - & 8 & 14 & 13 & 13.10 & (41.1) & 0.29 & 0.36\end{array}$

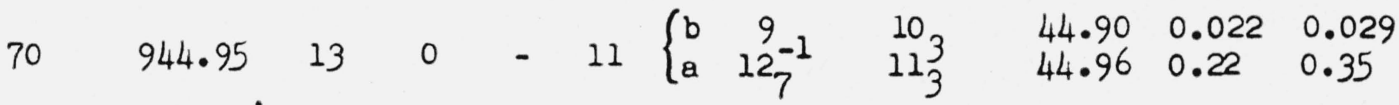

$\begin{array}{lllllllllllll}- & 946.73^{\dagger} & 6 & 0 & - & 0 & \text { a } & 8 & 7 & 7_{-1} & 46.73 & 0.13 & 0.04\end{array}$

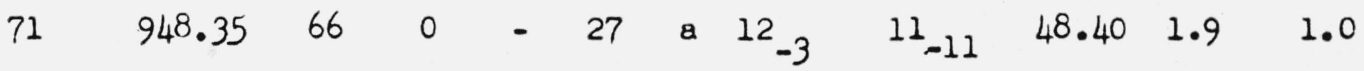

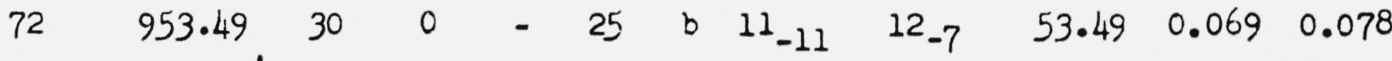
$\begin{array}{lllllllllllll}73 & 954.17^{\dagger} & 7 & 0 & - & 6 & b & 8 & 6 & 95 & 53.99 & 0.014 & 0.013\end{array}$

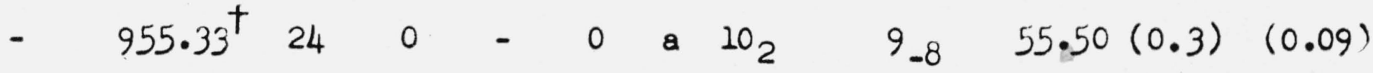

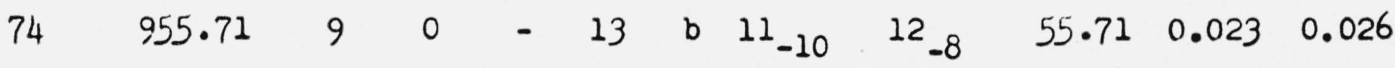

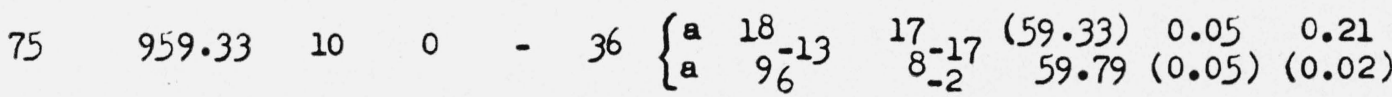

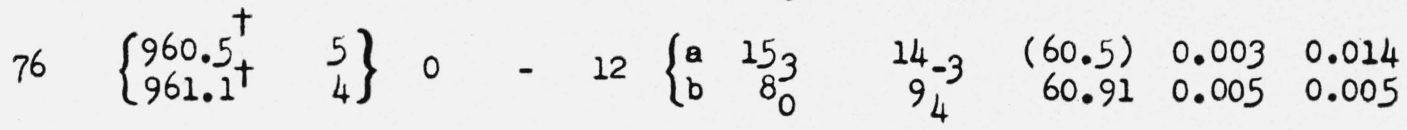

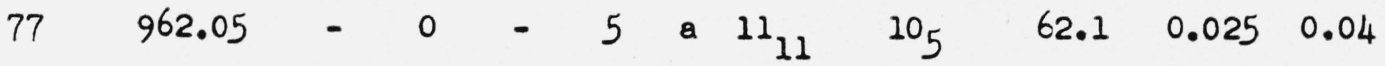

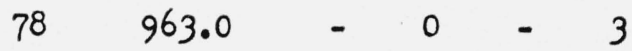

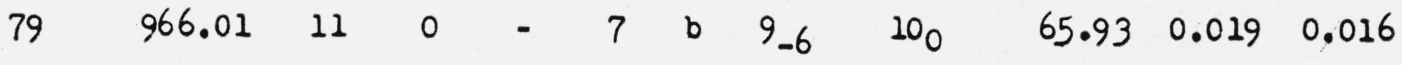

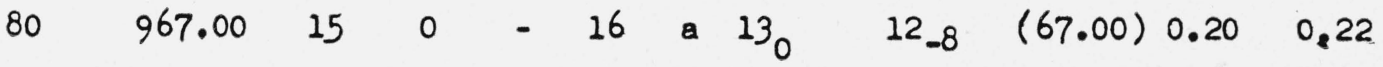


TABLE I-Continued.

\begin{tabular}{|c|c|c|c|c|c|c|c|c|}
\hline \multirow{4}{*}{ 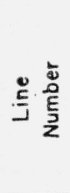 } & \multicolumn{4}{|c|}{ OBSERVED DATA } & \multirow{4}{*}{$\begin{array}{l}\text { Identification } \\
J_{\tau}^{\prime}-J_{\tau}^{\prime \prime}\end{array}$} & \multicolumn{3}{|c|}{ CALCULATED DATA } \\
\hline & \multirow{3}{*}{$\begin{array}{c}\text { Wave } \\
\text { Number } \\
\mathrm{cm}^{-1} \text { (vac) }\end{array}$} & \multicolumn{3}{|c|}{ Intensity } & & \multirow{3}{*}{$\begin{array}{c}\text { Wove } \\
\text { Number } \\
\mathrm{cm}^{-1} \text { (voc) }\end{array}$} & \multicolumn{2}{|c|}{ Intensity } \\
\hline & & \multirow{2}{*}{ Solar } & & boratory & & & & \\
\hline & & & $\begin{array}{l}\text { Air } \\
\text { only }\end{array}$ & 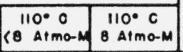 & & & $14^{\circ} \mathrm{C}$ & $110^{\circ} \mathrm{c}$ \\
\hline
\end{tabular}

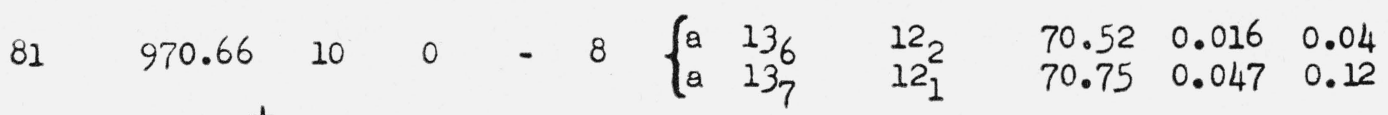

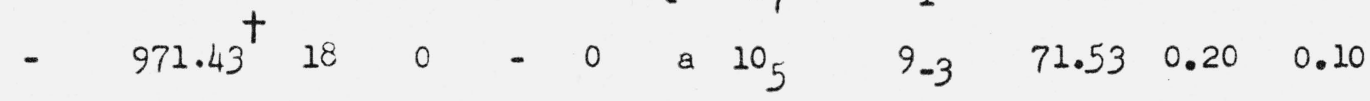
$82 \quad 971.75 \quad 12 \quad 0 \quad-20$

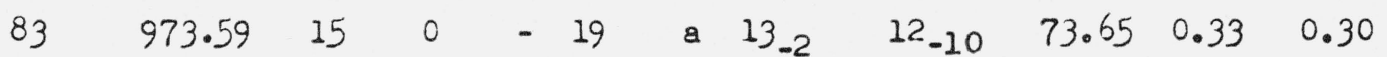

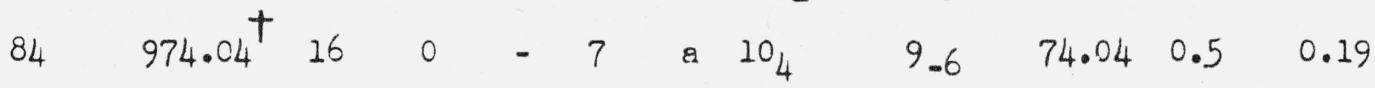
$\begin{array}{lllllllllllll}85 & 976.07 & 40 & 0 & -24 & 24 & 13 & -5 & 12 & -11 & 76.07 & 0.7 & 0.5\end{array}$ $\begin{array}{llllllllllllll}86 & 977.54 & 15 & 0 & - & 8 & 0 & 8 & -7 & 9 & -1 & 77.28 & 0.084 & 0.045\end{array}$

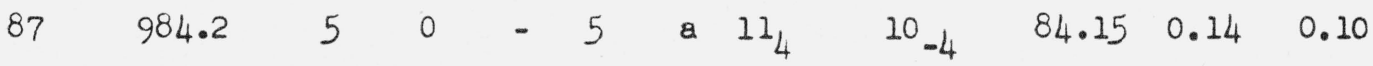
$\begin{array}{lllllllllllll}88 & 990.3 & 6 & 0 & - & 3 & \text { a } & 129 & 11_{5} & (90.3) & 0.007 & 0.015\end{array}$ $89 \quad 994.4 \quad 10 \quad 0 \quad-4$

$\begin{array}{lllllllllllllllll}90 & 998.87 & 30 & 0 & - & 10 & b & 8 & -3 & 9 & 98.70 & 0.080 & 0.026\end{array}$

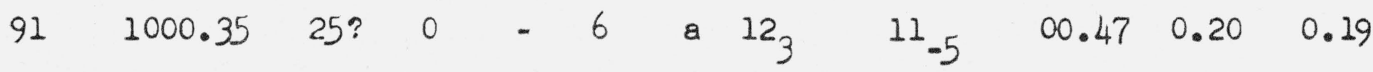
$92 \quad 1003.8 \quad 10 ? \quad 0 \quad-\quad 3$ a $97 \quad 8_{-3} 03.85(0.40)(0.14)$

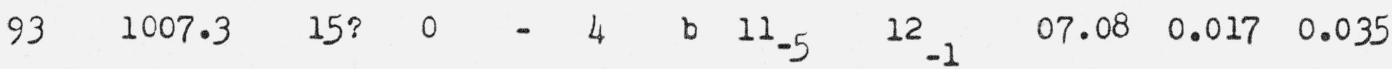
$\begin{array}{llllllllllllllll}94 & 1010.12 & 15 & 0 & -16 & \mathrm{~b} & 10 & -10 & 11.6 & 09.96 & 0.11 & 0.085\end{array}$

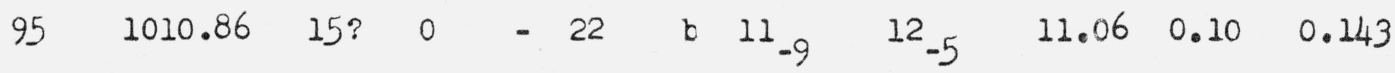
$\begin{array}{llllll}96 & 1011.64 & 10 ? & 0 & - & \end{array}$ $\begin{array}{lllllllllllllll}97 & 1014.56 & 50 ? & 0 & - & 38 & \mathrm{~b} & 10 & -9 & 11-7 & 14.38 & 0.318 & 0.265\end{array}$ $\begin{array}{lllllllllllllll}98 & 1017.6 & 20 ? & 0 & - & 15 & \mathrm{~b} & 8_{-5} & 9_{1} & 17.79 & 0.202 & 0.128\end{array}$ $99 \quad 1017.9 \quad 30 ? \quad 0 \quad-17 \quad$ a $14-1 \quad 13-9 \quad(17.9) \quad 0.16 \quad 0.22$ $\begin{array}{llllllllllll}100 & 1019.5 & 5 ? & 0 & - & 2 & \mathrm{~b} & 10_{-4} & 110 & 19.52 & 0.013 & 0.018\end{array}$ 
TABIE I-Continued.

\begin{tabular}{|c|c|c|c|c|c|c|c|c|}
\hline \multirow{4}{*}{ 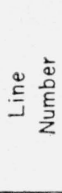 } & \multicolumn{4}{|c|}{ OBSERVED DATA } & \multirow{4}{*}{$\begin{array}{l}\text { Identification } \\
J_{\tau}^{\prime}-J_{\tau}^{\prime \prime}\end{array}$} & \multicolumn{3}{|c|}{ CALCULATED DATA } \\
\hline & \multirow{3}{*}{$\begin{array}{c}\text { Wave } \\
\text { Number } \\
\mathrm{cm}^{-1} \text { (vac) }\end{array}$} & \multicolumn{3}{|c|}{ Intensity } & & \multirow{3}{*}{$\begin{array}{c}\text { Wave } \\
\text { Number } \\
\mathrm{cm}^{-1} \text { (vac) }\end{array}$} & \multicolumn{2}{|c|}{ Intensity } \\
\hline & & \multirow{2}{*}{ Solar } & & boratory & & & & \\
\hline & & & $\begin{array}{l}\text { Air } \\
\text { only }\end{array}$ & \begin{tabular}{|c|c|}
$110^{\circ} \mathrm{c}$ & $110^{\circ} \mathrm{C}$ \\
8 Atmo-M & 8 Atmo-M \\
\end{tabular} & & & $14^{\circ} \mathrm{C}$ & $110 \cdot \mathrm{c}$ \\
\hline
\end{tabular}

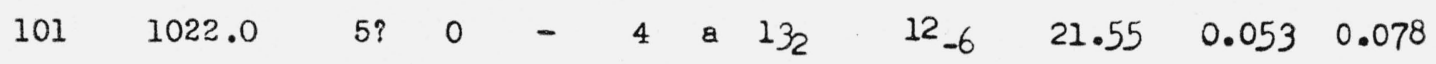
$102 \quad 1028.4730 ? \quad-13\left\{\begin{array}{llllll}\mathrm{b} & { }^{7}-2 & 84 & 28.47 & 0.043 & 0.024 \\ \mathrm{a} & 11_{3}^{-2} & 10_{-7} & 28.45 & 0.5 & 0.25\end{array}\right.$

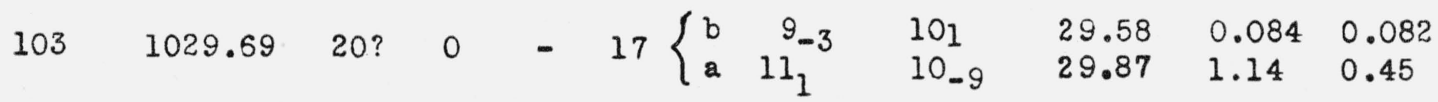
$\begin{array}{lllllllllll}104 & 1030.58 \quad 10 ? \quad 0 \quad 13 \quad b^{11}-8 & 12 & -6 & 30.0 & 0.033 & 0.048\end{array}$ $105 \quad 1032.76 \quad 20 ? \quad 0 \quad-\quad 5$

$106 \quad 1039.53 \quad 0 \quad-30\left\{\begin{array}{cccccc}\mathrm{b} & 8-2 & 92 & 39.31 & 0.043 & 0.034 \\ \mathrm{~b} & 11-7 & 12_{-3} & 39.35 & 0.075 & 0.128\end{array}\right.$ $\begin{array}{lllllllllllll}107 & 1042.57 \quad 15 ? \quad 0 \quad 17 \quad 2 \quad 14-3 \quad 13-11 \quad(42.5) & 0.22 & 0.28\end{array}$ $108 \quad 1044.4 \quad ? \quad 0 \quad-\quad 3$

$\begin{array}{lllllllllllll}109 & 1049.39 & ? & 0 & - & 7 & \mathrm{~b} & 7_{-6} & 8_{0} & 49.39 & 0.13 & 0.052\end{array}$ $110 \quad 1050.22 \quad ? \quad 0 \quad 6$

$\begin{array}{lllllllllllll}111 & 1051.28 & ? & 0 & - & 11 & \mathrm{~b} & 7 & -1 & 83 & 51.28 & 0.176 & 0.096\end{array}$

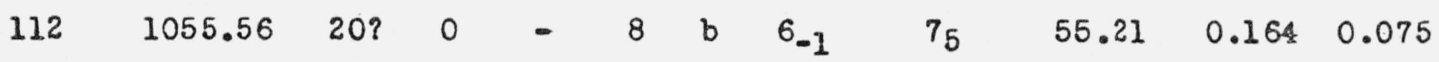

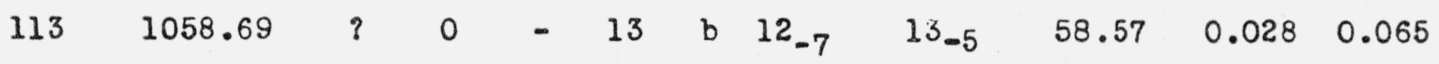

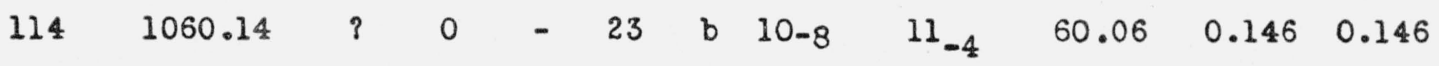
$\begin{array}{llllllllllll}115 & 1062.61 & ? & 0 & - & 8 & b & 7_{-4} & 8_{2} & 62.50 & 0.154 & 0.075\end{array}$ $116 \quad 1063.6 \quad ? \quad 0 \quad-\quad 3$

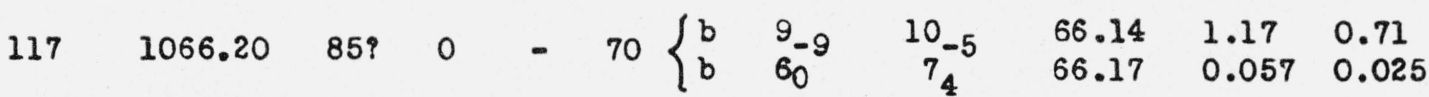
$\begin{array}{llllllllllll}118 & 1072.69 & ? & 0 & - & 15 & \mathrm{~b} & 10_{-6} & 11-2 & 72.52 & 0.084 & 0.10\end{array}$ $119 \quad 1074.4670 ? \quad 0 \quad-\quad 41 \quad b \quad 9_{-8} \quad 0_{-6} \quad 74.31 \quad 0.415 \quad 0.25$ $\begin{array}{llllllllllllll}120 & 1085.4 & ? & 0 & - & 3 & b & 5_{1} & 6_{5} & 85.37 & 0.096 & 0.035\end{array}$ 
TABLE 1 -Continued.

\begin{tabular}{|c|c|c|c|c|c|c|c|c|}
\hline \multirow{4}{*}{ 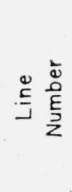 } & \multicolumn{4}{|c|}{ OBSERVED DATA } & \multirow{4}{*}{$\begin{array}{l}\text { Identification } \\
J_{\tau}^{\prime}-J_{\tau}^{\prime \prime}\end{array}$} & \multicolumn{3}{|c|}{ CALCULATED DATA } \\
\hline & \multirow{3}{*}{$\begin{array}{c}\text { Wave } \\
\text { Number } \\
\mathrm{cm}^{-1} \text { (vac) }\end{array}$} & \multicolumn{3}{|c|}{ Intensity } & & \multirow{3}{*}{$\begin{array}{c}\text { Wave } \\
\text { Number } \\
\mathrm{cm}^{-1} \text { (vac) }\end{array}$} & \multicolumn{2}{|c|}{ Intensity } \\
\hline & & \multirow{2}{*}{ Solar } & & aboratory & & & & \\
\hline & & & $\begin{array}{l}\text { Air } \\
\text { only }\end{array}$ & \begin{tabular}{|c|c|}
$110^{\circ} \mathrm{C}$ & $110^{\circ} \mathrm{C}$ \\
$8 \mathrm{~A}^{\mathrm{A} m \mathrm{~m}-\mathrm{M}}$ & 8 Atmo-M \\
\end{tabular} & & & $14^{\circ} \mathrm{C}$ & $110 \cdot \mathrm{c}$ \\
\hline
\end{tabular}

$\begin{array}{llllll}121 & 1088.1 & ? & 0 & - & 2\end{array}$

$\begin{array}{llllllllllll}122 & 1091.24 & 82 & 0 & - & 55 & \mathrm{~b} & 10_{-7} & 11_{-5} & 91.21 & 0.575 & 0.54\end{array}$

$\begin{array}{llllllllllllll}123 & 1099.74 & 72 & 0 & - & 54 & \text { b } & 9_{-5} & 10_{-1} & 99.04 & 0.665 & 0.55\end{array}$

$\begin{array}{lllllllllllll}124 & 1101.47 & 76 & 0 & - & 34 & \mathrm{~b} & 6_{-3} & 7_{3} & 01.42 & 0.85 & 0.32\end{array}$

$\begin{array}{llllll}125 & 1105.3 \quad 5 ? \quad 0 \quad 8\end{array}$

$\begin{array}{llllllllllll}126 & 1106.76 & 83 & 0 & - & 78 & \mathrm{~b} & 9_{-7} & 10_{-3} & 06.73 & 1.60 & 1.15\end{array}$

$\begin{array}{lllllllllllll}127 & 1111.59 & 82 & 0 & - & 41 & \mathrm{~b} & 6 & -5 & 7_{1} & 11.50 & 1.21 & 0.41\end{array}$

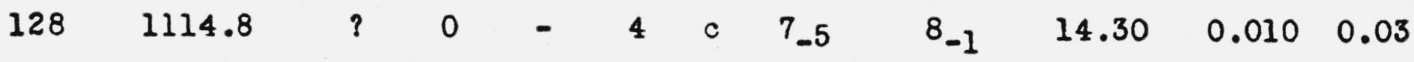

$\begin{array}{llllllllllll}129 & 1117.71 & 23 ? & 0 & - & 19 & \mathrm{~b} & 11_{-6} & 12_{-4} & 17.60 & 0.063 & 0.098\end{array}$

$\begin{array}{lllllllllll}130 & 1120.9 & 84 & 0 & 14 \\ 76 & b & 8_{-4} & 9_{0} & 20.76 & 0.45 & 0.29\end{array}$

$\left.\begin{array}{llllllllll}131 & 1121.24 & 89 & 0 & 21\end{array}\right\}^{76} \quad \begin{array}{lllll}\text { b }_{-8} & 9_{-4} & 21.24 & 1.37 & 0.62\end{array}$

$\begin{array}{lllllllllllll}132 & 1135.80 & 98 & 0 & 44 & 86 & \text { b } & 8 & -7 & 9_{-5} & 35.80 & 1.78 & 0.80\end{array}$

$\begin{array}{llllllllllll}133 & 1137.46 & 85 & 0 & 16 & 65 & \mathrm{~b} & 7_{-3} & 8_{1} & 37.19 & 1.63 & 0.78\end{array}$

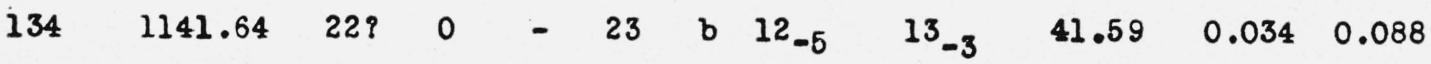

$\begin{array}{llllllllllll}135 & 1149.48 & 77 & 0 & 22 & 78 & \text { b } & 8_{-6} & 9_{-2} & 49.39 & 1.82 & 0.98\end{array}$

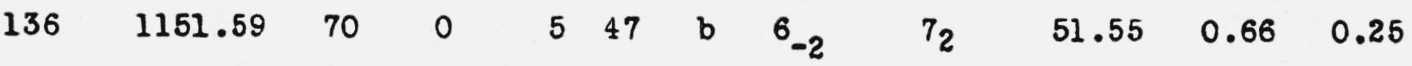

$\begin{array}{llllllllllll}137 & 1152.44 & 77 & 0 & 19 & 72 & \text { b } & 9_{-6} & 10_{-4} & 52.42 & 0.91 & 0.63\end{array}$

$\begin{array}{llllllllllll}138 & 1165.27^{\dagger} & 87 & 0 & 29 & 59 & \mathrm{~b} & 5_{-4} & 6_{2} & 65.10 & 0.86 & 0.22\end{array}$

$\left.\begin{array}{lll}139 & 1165.4^{\dagger} & 83 \\ 1165.9 & 73\end{array}\right\} \quad 0 \quad 1535\left\{\begin{array}{lllllll}b & 5-1 & 6_{3} & 65.40 & 1.56 & 0.48 \\ b & 4-1 & 5_{5} & 65.84 & 0.59 & 0.145\end{array}\right.$

$\begin{array}{llllll}140 & 1167.04 & ? & 0 & 5 & 16\end{array}\left\{\begin{array}{llllll}c & 8-5 & 9-3 & 66.8 & 0.009 & 0.032 \\ 0 & 5_{-3} & 6_{1} & 67.1 & 0.014 & 0.030\end{array}\right.$ 
TABLE I-Continued.

\begin{tabular}{|c|c|c|c|c|c|c|c|c|}
\hline \multirow{4}{*}{ 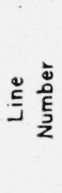 } & \multicolumn{4}{|c|}{ OBSERVED DATA } & \multirow{4}{*}{$\begin{array}{l}\text { Identification } \\
J_{\tau}^{\prime}-J_{\tau}^{\prime \prime}\end{array}$} & \multicolumn{3}{|c|}{ CALCULATED DATA } \\
\hline & \multirow{3}{*}{$\begin{array}{c}\text { Wove } \\
\text { Number } \\
\mathrm{cm}^{-1} \text { (voc) }\end{array}$} & \multicolumn{3}{|c|}{ Intensity } & & \multirow{3}{*}{$\begin{array}{l}\text { Wave } \\
\text { Number } \\
\mathrm{cm}^{-1} \text { (vac) }\end{array}$} & \multicolumn{2}{|c|}{ Intensity } \\
\hline & & \multirow[b]{2}{*}{ Solor } & & boratory & & & & \\
\hline & & & $\begin{array}{l}\text { Air } \\
\text { only }\end{array}$ & \begin{tabular}{|c|c|}
$110^{\circ}$ & 0 \\
$\theta^{\prime}$ Atmo-M & $110^{\circ} \mathrm{C}$ \\
OAtmo-M
\end{tabular} & & & $14^{\circ} \mathrm{c}$ & $110 . \mathrm{c}$ \\
\hline
\end{tabular}

$\begin{array}{llllll}141 & 1169.4 & ? & 0 & -\end{array}$

$\begin{array}{lllllllllllll}142 & 1171.4 & ? & 0 & - & 11 & b & 9_{-8} & 9_{-2} & 71.47 & 0.043 & 0.021\end{array}$

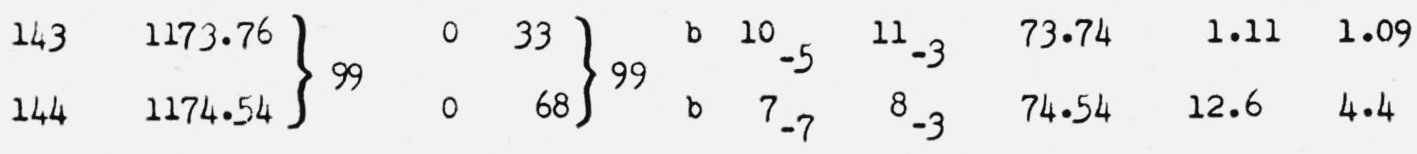

$145 \quad 1178.5515 ? \quad 0 \quad-15$

$\begin{array}{llllllllllll}146 & 1180.75 & 55 ? & 0 & - & 9 & \mathrm{~b} & 4_{0} & 5_{4} & 80.94 & 0.29 & 0.06\end{array}$

$\begin{array}{llllll}147 & 1182.2 & 3 & 0 & - & 3\end{array}$

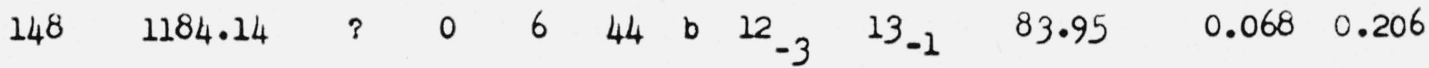

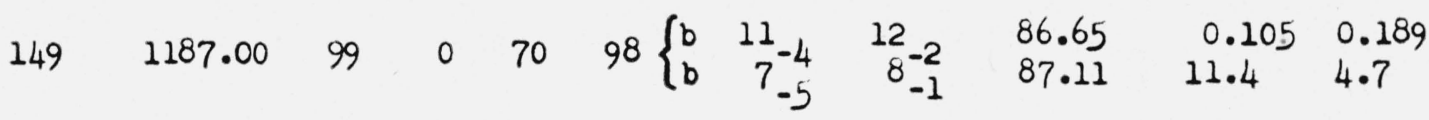

$150 \quad 1191.16 \quad 35 ? \quad 0 \quad-9$

$151 \quad 1193.6728 ? 0 \quad-9$

$\begin{array}{lllllllllllll}152 & 1195.41 & ? & 0 & 6 & 27 & \mathrm{~b} & 12 & -1 & 13_{1} & 95.28 & 0.046 & 0.196\end{array}$

$\begin{array}{llllllllllll}153 & 1198.22 & 98 & 0 & 48 & 95 & \mathrm{~b} & 7.6 & 8_{-4} & 98.18 & 5.2 & 1.78\end{array}$

$\begin{array}{llllll}154 & 1200.80 & ? & 0 & 2 & 17\end{array}$

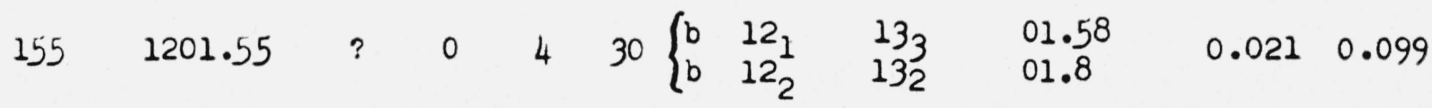

$\left.156\left\{\begin{array}{l}1206.07^{\dagger} \\ 1206.37^{\dagger}\end{array} \frac{523}{30 ?}\right\}\right\}_{0}-13$

$\begin{array}{llllllllllll}157 & 1207.35 & 0 & - & 13 & 5 & 5 & 6_{-5} & 07.5 & 0.048 & 0.082\end{array}$

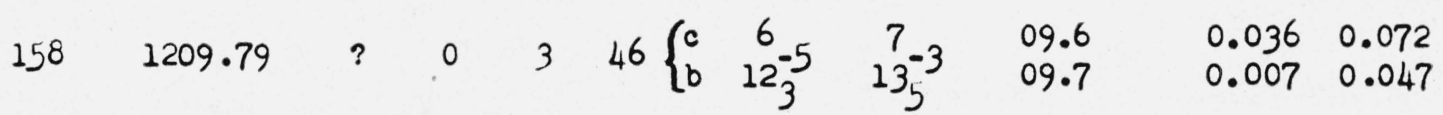

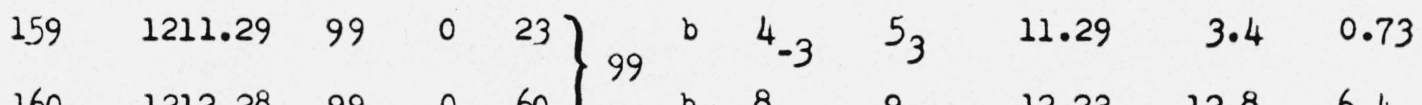

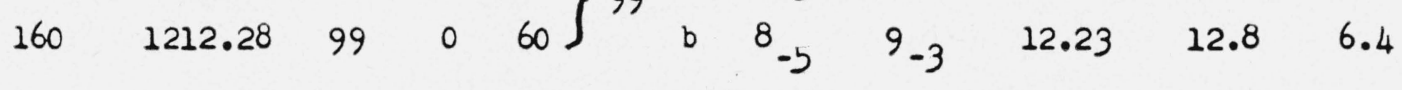


TABLE I-Continued.

\begin{tabular}{|c|c|c|c|c|c|c|c|c|}
\hline \multirow{4}{*}{ 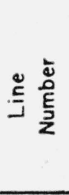 } & \multicolumn{4}{|c|}{ OBSERVED DATA } & \multirow{4}{*}{$\begin{array}{l}\text { Identification } \\
J_{\tau}^{\prime}-J_{\tau}^{\prime \prime}\end{array}$} & \multicolumn{3}{|c|}{ CALCULATED DATA } \\
\hline & \multirow{3}{*}{$\begin{array}{c}\text { Wave } \\
\text { Number } \\
\mathrm{cm}^{-1} \text { (voc) }\end{array}$} & \multicolumn{3}{|c|}{ Intensity } & & \multirow{3}{*}{$\begin{array}{c}\text { Wave } \\
\text { Number } \\
\mathrm{cm}^{-1} \text { (vac) }\end{array}$} & \multicolumn{2}{|c|}{ Intensity } \\
\hline & & \multirow{2}{*}{ Solor } & & aboratory & & & & \\
\hline & & & Alr & \begin{tabular}{|c|c|}
$110^{\circ} 0$ & 0 \\
$8_{0}$ Atmo-M & $110^{\circ} \mathrm{C}$ Atmo-M \\
\end{tabular} & & & $14^{\circ} \mathrm{C}$ & $110 . \mathrm{c}$ \\
\hline
\end{tabular}

$\begin{array}{llllllllllll}161 & 1213.0 & ? & 0 & 19 & 99 & \mathrm{~b} & 11 & 12 & 13.00 & 0.10 & 0.22\end{array}$

$\begin{array}{lllllllllllll}162 & 1214.91 & ? & 0 & 3 & 25 & \mathrm{~b} & 8_{-7} & 8_{-1} & 15.06 & 0.36 & 0.15\end{array}$

$\left.\begin{array}{lllll}163 & 1218.63 & 98 & 0 & 40\end{array}\right\}_{90} \begin{array}{lllllll}\mathrm{b} & 6_{-4} & 7_{0} & 18.63 & 6.70 & 2.07\end{array}$

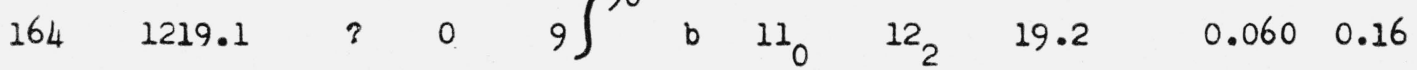

$\begin{array}{llllllllllll}165 & 1220.43 & ? & 0 & 18 & 70 & \mathrm{~b} & 11_{1} & 12_{1} & 20.60 & 0.18 & 0.47\end{array}$

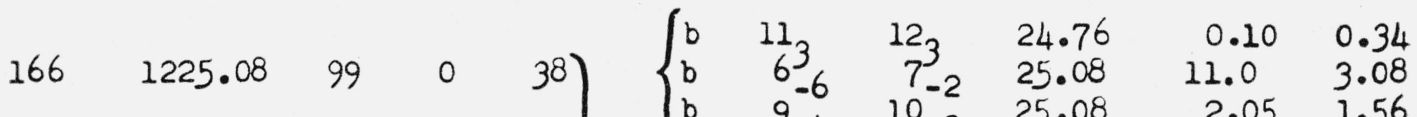

$\left.\begin{array}{llll}167 & 1225.5 & 99 & 0\end{array}\right\} \begin{array}{lllllll} & 9 & -4 & 10_{-2} & 25.08 & 2.05 & 1.56 \\ 99 & \mathrm{~b} & 10_{-3} & 11-1 & 25.46 & 1.97 & 2.36\end{array}$

$\begin{array}{lllllllllll}168 & 1226.1 & 78 & 0 & 9 & b & 11-1 & 12 & 26.06 & 0.355 & 0.74\end{array}$

$\begin{array}{llllll}169 & 1228.53 \quad & 3 & 0 & - & 13\end{array}$

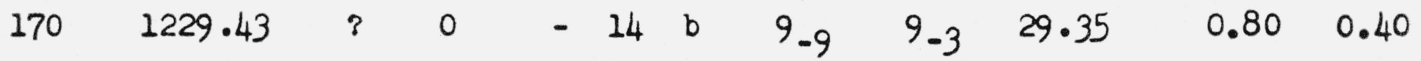

$171 \quad 1232.1 \quad ? \quad 0 \quad-\quad 3$

$\begin{array}{lllllllllllll}172 & 1233.31 & ? & 0 & - & 47 & \mathrm{~b} & 11_{5} & 12_{5} & 32.88 & 0.041 & 0.18\end{array}$

$\begin{array}{lllll}173 & 1235.23 & ? & 0 & -19\end{array}$

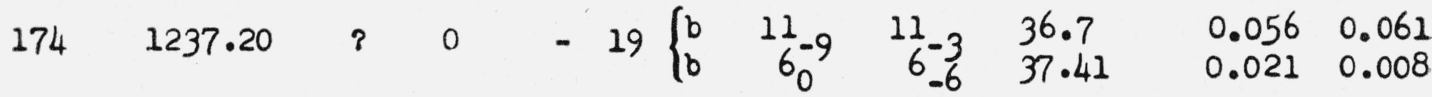

$\begin{array}{llllllllllll}175 & 1239.25 & ? & 0 & 25 & 98 & \mathrm{~b} & 10_{-1} & 11_{1} & 39.19 & 1.50 & 2.17\end{array}$

$\begin{array}{lllll}176 & 1240.6 \quad ? \quad 0 \quad-35\end{array}$

$\begin{array}{lllll}177 & 1242.90 & 953 & 0 & 17\end{array} 98\left\{\begin{array}{llllll}b & 10 & 11 & 42.75 & 1.17 & 2.11 \\ b & 102 & 113 & 43.1 & 0.39 & 0.70\end{array}\right.$

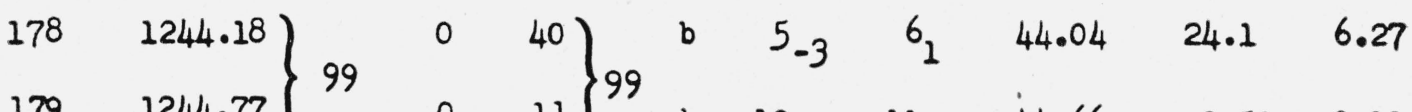

$\left.\left.\begin{array}{llll}179 & 1244.77\end{array}\right\} 99 \quad 11\right\} \begin{array}{lllllll}99 & & & & & & \\ & b & 10_{0} & 110 & 44.66 & 0.52 & 0.99\end{array}$

$\begin{array}{llllllllllllll}180 & 1246.63 & ? & - & 0 & 7 & b & 7 & & & 0 & 7 & & \end{array}$ 
TABLE I-Continued.

\begin{tabular}{|c|c|c|c|c|c|c|c|c|}
\hline \multirow{4}{*}{ : } & \multicolumn{4}{|c|}{ OBSERVED DATA } & \multirow{4}{*}{$\begin{array}{l}\text { Identification } \\
J_{\tau}^{\prime}-J_{\tau}^{\prime \prime}\end{array}$} & \multicolumn{3}{|c|}{ CALCULATED DATA } \\
\hline & \multirow{3}{*}{$\begin{array}{c}\text { Wave } \\
\text { Number } \\
\mathrm{cm}^{-1} \text { (vac) }\end{array}$} & \multicolumn{3}{|c|}{ Intensify } & & \multirow{3}{*}{$\begin{array}{c}\text { Wove } \\
\text { Number } \\
\mathrm{cm}^{-1} \text { (vac) }\end{array}$} & \multicolumn{2}{|c|}{ Intensity } \\
\hline & & \multirow{2}{*}{ Solor } & \multicolumn{2}{|c|}{ Laboratory } & & & & \\
\hline & & & $\begin{array}{l}\text { Alr } \\
\text { only }\end{array}$ & 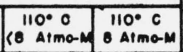 & & & $14^{\circ} \mathrm{C}$ & $110 \cdot c$ \\
\hline
\end{tabular}

$\begin{array}{llllllllllll}181 & 1248.52 & ? & - & 11 & 86 & \mathrm{~b} & 10 & 11_{5} & 48.49 & 0.46 & 1.06\end{array}$

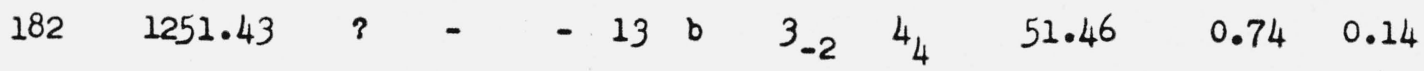
$\begin{array}{llllll}183 & 1252.44 & ?\end{array}$

$\begin{array}{lllllllllllll}184 & 1253.67 & ? & - & 0 & 5 & b & 7_{-6} & 7_{0} & 53.51 & 0.43 & 0.14\end{array}$

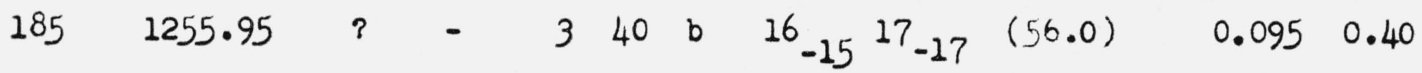
$\begin{array}{lllllll}186 & 1257.07 & 6 & 6\end{array}\left\{\begin{array}{llllll}\mathrm{b} & 10_{5} & 11 & 57.07 & 0.182 & 0.56 \\ \mathrm{~b} & 11_{-3} & 12^{7} & 57.10 & 0.645 & 1.10\end{array}\right.$

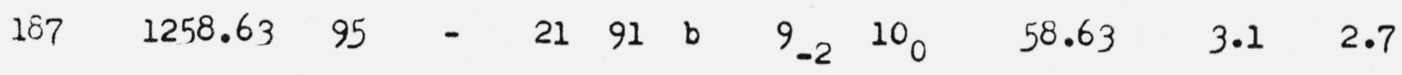
$\begin{array}{llllllllllll}188 & 1260.38 & 99 & - & 67 & 99 & \mathrm{~b} & 6_{-5} & 7_{-3} & 60.32 & 53.7 & 14.5\end{array}$ $\begin{array}{llllllllllll}189 & 1264.04 & ? & - & 22 & 92 & \mathrm{~b} & 9_{0} & 10_{2} & 63.93 & 2.0 & 2.1\end{array}$

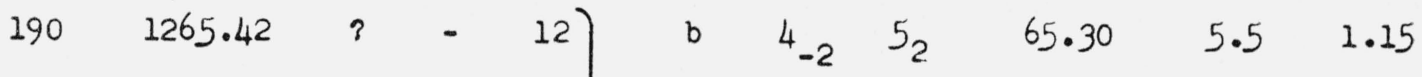
$\begin{array}{llllllllllll}191 & 1266.11 & ? & - & 41 & b & 9_{1} & 10_{1} & 66.31 & 6.2 & 6.5\end{array}$ $\begin{array}{llllllllll}192 & 1266.63 & - & 39 & b & 9_{3} & 10_{3} & 66.55 & 4.2 & 4.5\end{array}$ $193 \quad 1268.4099-49\left\{\begin{array}{cccccc}\mathrm{b} & 10 & 11 & 67.83 & 0.90 & 1.02 \\ \mathrm{~b} & 10^{-2} & 11^{-2} & 67.93 & 0.06 & 0.26 \\ \mathrm{~b} & 7 & 8_{-2}^{9} & 68.28 & 19.2 & 7.1\end{array}\right.$ $1941269.9799-60\} \begin{array}{lllllll}99 & \mathrm{~b} & 8^{-4} & 9_{-1} & 69.72 & 31.1 & 17.0\end{array}$ $\begin{array}{lllllllllll}195 & 1271.80 & 99 & - & 61 & \mathrm{~b} & 5_{-5} & 6_{-1} & 71.73 & 73 & 16.3\end{array}$

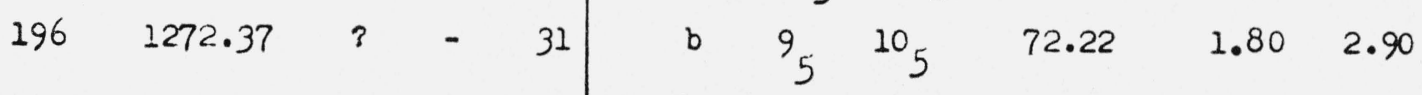

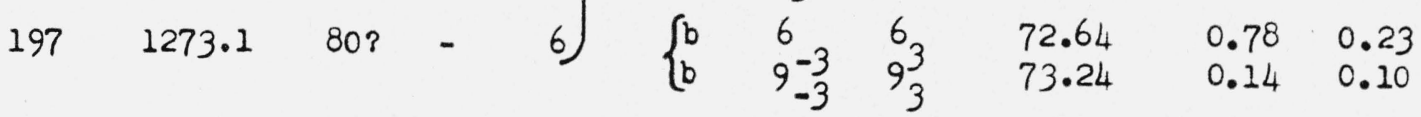

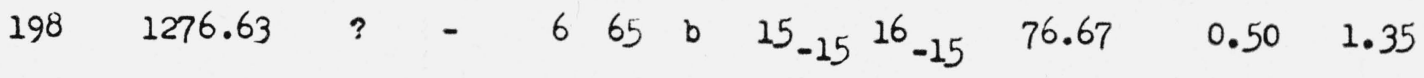
$\begin{array}{llllllllllll}199 & 1280.09 & 99 & - & 48 & \text { b } & 9_{-1} & 10_{-1} & 80.09 & 10.4 & 8.88\end{array}$ $\begin{array}{lllllllllllll}200 & 1281.22 & 85 ? & - & 20 & 89 & \mathrm{~b} & 9_{7} & 107 & 80.99 & 1.06 & 2.30\end{array}$ 
TABLE I-Continued.

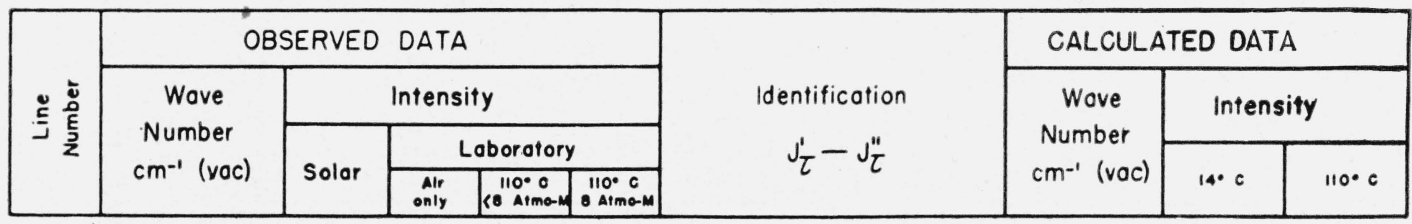

$201 \quad 1282.9 \quad ? \quad-\quad 37$

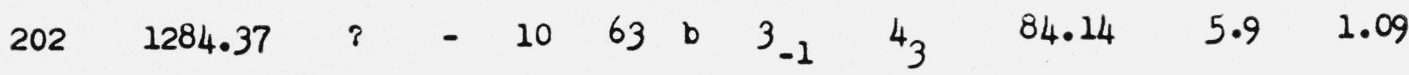

$203 \quad 1284.89 \quad 3 \quad-\quad 0 \quad 40$

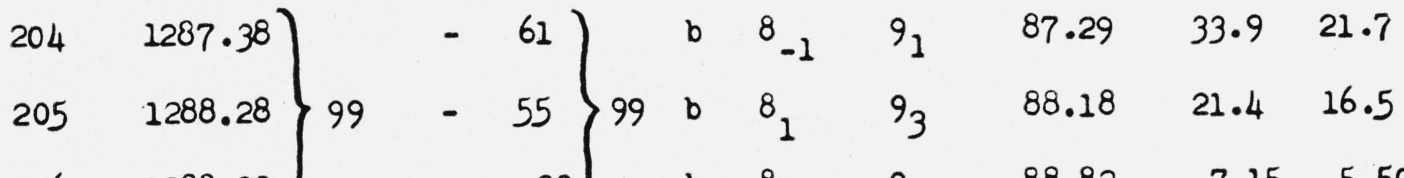

$\left.\begin{array}{lllllllll}206 & 1288.92\end{array}\right] \quad \begin{array}{llllll}33 & 8_{2} & 9_{2} & 88.82 & 7.15 & 5.50\end{array}$

$\begin{array}{llllllllllll}207 & 1290.59 & ? & - & 47 & 98 & \mathrm{~b} & 8_{3} & 9_{5} & 90.52 & 14.6 & 18.2\end{array}$

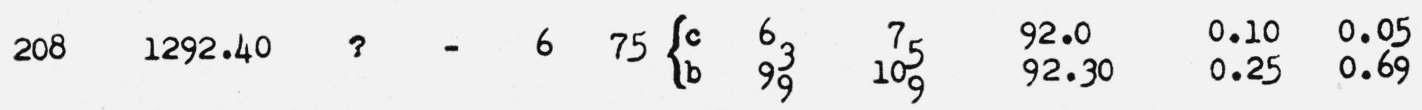

$209 \quad 1296.67 \quad-5599\left\{\begin{array}{llllll}b & 8_{5} & 9 & 96.59 & 6.25 & 7.81 \\ b & 8_{0} & 9 & 96.53 & 12.1 & 7.6\end{array}\right.$

$\begin{array}{llllllllllll}210 & 1300.9 & ? & - & 0 & 2 & \mathrm{c} & 6_{5} & 77 & 01.0 & 0.04 & 0.19\end{array}$

$211 \quad 1302.7 \quad ? \quad-\quad 0 \quad 2$

$2121304.46 \quad ? \quad-\quad 0 \quad 22$

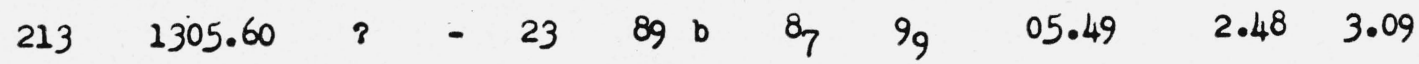

$214 \quad 1306.31 \quad ? \quad-\quad 5 \quad 54$

$\begin{array}{lllllllllllll}215 & 1308.25 & ? & - & 55 & 96 & \mathrm{~b} & 7_{-2} & 8_{0} & 08.18 & 42.0 & 17.4\end{array}$

$\begin{array}{lllllllllllllllllll}216 & 1312.61 & ? & - & 75 & - & b & 7_{3} & 8_{3} & 12.71 & 88 & 52\end{array}$

$217 \quad 1313.64 \quad-64-\left\{\begin{array}{llllll}b & 4 & 50 & 13.35 & 39 & 7.4 \\ b & 70 & 82 & 13.68 & 36.7 & 17.6\end{array}\right.$

$\begin{array}{llllllllllllll}218 & 1314.82 & ? & - & 59 & - & \mathrm{b} & 7_{5} & 8_{5} & 14.73 & 43.8 & 31.9\end{array}$

$\begin{array}{llllllllllllll}219 & 1316.20 & 3 & - & 32 & - & \mathrm{b} & 11 & 12 & 12 & -5 & 16.38 & 1.67 & 2.39\end{array}$

$\begin{array}{llllllllllll}220 & 1317.04 & ? & - & 75 & - & \mathrm{b} & 7_{1} & 8_{1} & 16.92 & 113 & 54\end{array}$ 
TABLE "I-Continued.

\begin{tabular}{|c|c|c|c|c|c|c|c|c|}
\hline \multirow{4}{*}{ 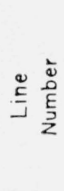 } & \multicolumn{4}{|c|}{ OBSERVED DATA } & \multirow{4}{*}{$\begin{array}{l}\text { Identification } \\
\qquad J_{\tau}^{\prime}-J_{\tau}^{\prime \prime}\end{array}$} & \multicolumn{3}{|c|}{ CALCULATED DATA * } \\
\hline & \multirow{3}{*}{$\begin{array}{c}\text { Wave } \\
\text { Number } \\
\mathrm{cm}^{-1} \text { (vac) }\end{array}$} & \multicolumn{3}{|c|}{ Intensity } & & \multirow{3}{*}{$\begin{array}{c}\text { Wave } \\
\text { Number } \\
\mathrm{cm}^{-1} \text { (vac) }\end{array}$} & \multicolumn{2}{|c|}{ Intensity } \\
\hline & & \multirow{2}{*}{ Solar } & \multicolumn{2}{|c|}{ Laboratory } & & & & \\
\hline & & & $\begin{array}{l}\text { Air } \\
\text { only }\end{array}$ & \begin{tabular}{|c|c|}
$110^{\circ} \mathrm{c}$ & $110^{\circ} \cdot \mathrm{c}$ \\
8 Atmo-M & 8 Atmo-M \\
\end{tabular} & & & $14^{\circ} \mathrm{c}$ & $110 . c$ \\
\hline
\end{tabular}

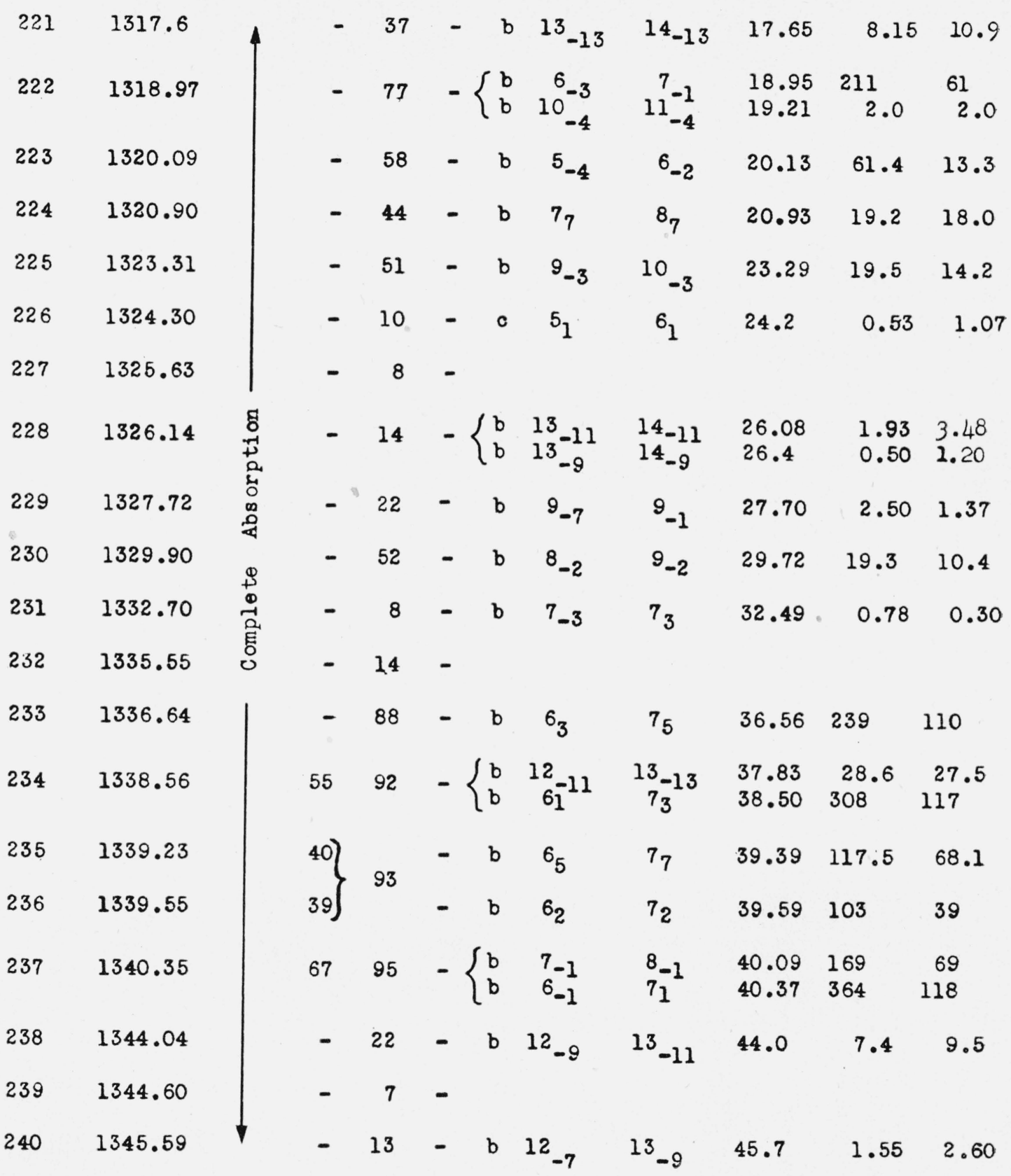


TABLE I-Continued.

\begin{tabular}{|c|c|c|c|c|c|c|c|c|}
\hline \multirow{4}{*}{ : } & \multicolumn{4}{|c|}{ OBSERVED DATA } & \multirow{4}{*}{$\begin{array}{l}\text { Identification } \\
J_{\tau}^{\prime}-J_{\tau}^{\prime \prime}\end{array}$} & \multicolumn{3}{|c|}{ CALCULATED DATA } \\
\hline & \multirow{3}{*}{$\begin{array}{c}\text { Wove } \\
\text { Number } \\
\mathrm{cm}^{-1} \text { (voc) }\end{array}$} & \multicolumn{3}{|c|}{ Intensity } & & \multirow{3}{*}{$\begin{array}{c}\text { Wove } \\
\text { Number } \\
\approx m^{-1} \text { (voc) }\end{array}$} & \multicolumn{2}{|c|}{ Intensity } \\
\hline & & \multirow{2}{*}{ Solor } & & aboratory & & & & \\
\hline & & & $\begin{array}{l}\text { Air } \\
\text { only }\end{array}$ & \begin{tabular}{|c|c|}
$110^{\circ}$ & 0 \\
$0^{11}$ Atmo-M & $110^{\circ}$ A Atmo-M \\
\end{tabular} & & & $14^{\circ} \mathrm{c}$ & $110 . \mathrm{c}$ \\
\hline
\end{tabular}

$\begin{array}{lllllllllll}241 & 1345.97 & - & 18 & - & 4 & 4 & 5_{3} & 46.0 & 1.22 & 2.04\end{array}$

$242 \quad 1347.01$

$243 \quad 1349.0$

$244 \quad 1349.39$

$245 \quad 1351.67$

$246 \quad 1352.41$

$247 \quad 1354.87$

$248 \quad 1356.0$

- $6-c 4_{2} 5_{2}$

47.1

0.410 .68

- 34 -

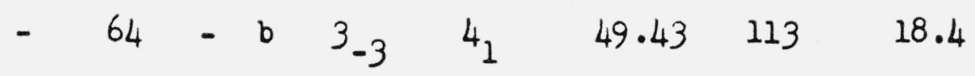

$-15-$

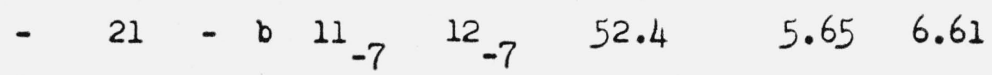

- $\begin{array}{llllllll}80 & - & b & 6 & 7 & 7 & 54.80 & 164\end{array}$

$-13-\left\{\begin{array}{llllll}c & 5 & 6 & 55.4 & 0.86 & 1.47 \\ b & 8_{-6}^{-1} & 8_{0}^{-1} & 56.54 & 2.6 & 1.08\end{array}\right.$

$249 \quad 1357.21$

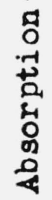

$250 \quad 1358.06$

13

- 55 -

$251 \quad 1361.09$

$70 \quad-\quad\left\{\begin{array}{cccccc}b & 11-9 & 12-9 & 61.02 & 19.5 & 18.1 \\ b & 55 & 65 & 61.09 & 570 & 211\end{array}\right.$

$252 \quad 1362.70$

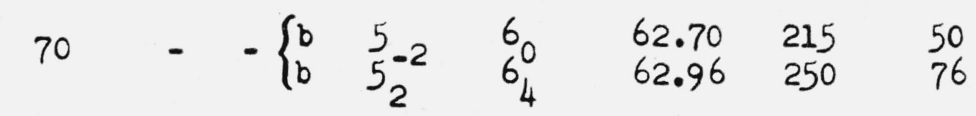

$253 \quad 1363.17$

$254 \quad 1365.9$

$255 \quad 1368.60$

$256 \quad 1369.78$

$257 \quad 1370.95$

$258 \quad 1372.28$

$259 \quad 1373.76$

$260 \quad 1375.09$

57

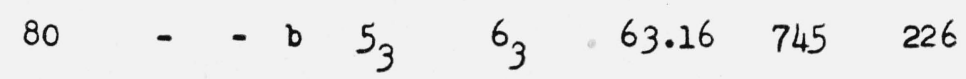

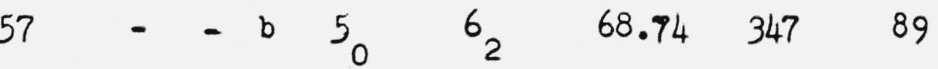

$680-\left\{\begin{array}{llllll}b & 12 & 12 & 69.89 & 3.8 & 3.5 \\ b & 1211 & 12-9 & 70.02 & 1.3 & 1.2\end{array}\right.$

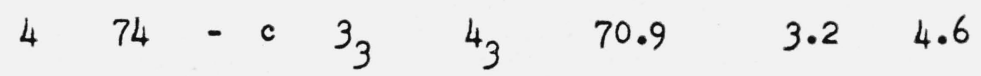

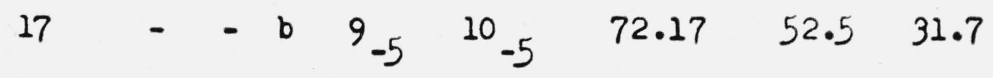

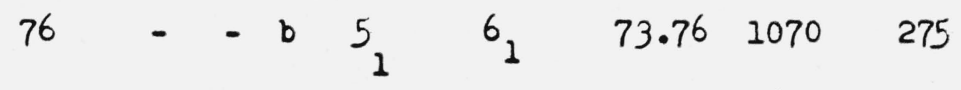

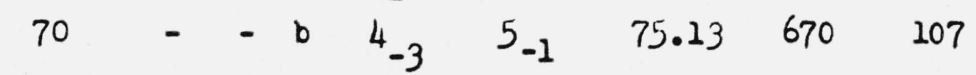


TABLE I-Continued.

\begin{tabular}{|c|c|c|c|c|c|c|c|c|c|}
\hline \multirow{4}{*}{ 冚 } & \multicolumn{5}{|c|}{ OBSERVED DATA } & \multirow{4}{*}{$\begin{array}{l}\text { Identification } \\
J_{\tau}^{\prime}-J_{\tau}^{\prime \prime}\end{array}$} & \multicolumn{3}{|c|}{ CALCULATED DATA } \\
\hline & \multirow{3}{*}{$\begin{array}{c}\text { Wave } \\
\text { Number } \\
\mathrm{cm}^{-1} \text { (vac) }\end{array}$} & \multicolumn{4}{|c|}{ Intensity } & & \multirow{3}{*}{$\begin{array}{c}\text { Wave } \\
\text { Number } \\
\mathrm{cm}^{-1} \text { (vac) }\end{array}$} & \multicolumn{2}{|c|}{ Intensity } \\
\hline & & \multirow{2}{*}{ Solar } & \multicolumn{3}{|c|}{ Laboratory } & & & & \\
\hline & & & $\begin{array}{c}\text { Alr } \\
\text { only }\end{array}$ & $\begin{array}{c}110^{\circ} \\
8 \\
8 \text { Atma-m }\end{array}$ & $\begin{array}{l}110^{\circ} \mathrm{c} \\
8 \text { Atmo-m }\end{array}$ & & & $14^{\circ} \mathrm{C}$ & $110^{\circ} \mathrm{c}$ \\
\hline
\end{tabular}

2611376.3

$262 \quad 1378.04$

$263 \quad 1378.5$

$264 \quad 1379.63$

$265 \quad 1382.11$

$266 \quad 1383.40$

$267 \quad 1384.26$

$268 \quad 1386.51$

$269 \quad 1387.55$

$270 \quad 1390.0$

$271 \quad 1390.95$

$272 \quad 1392.38$

$273 \quad 1394.50$

$274 \quad 1395.81$

$275 \quad 1397 \cdot 74$

$276 \quad 1399.16$

$277 \quad 1402.0$

$278 \quad 1403.54$

$279 \quad 1404.98$

28C $\quad 1407.09$
$3--$

$35-\quad\left\{\begin{array}{llllll}\mathrm{b} & 10 & 11-11 & 77.98 & 263 & 139 \\ \mathrm{~b} & 10_{-8}^{-9} & 111_{-8} & 78.07 & 19.5 & 13.3\end{array}\right.$

6 - $\begin{array}{lllllll}- & b & 7_{-5} & 7_{1} & 78.66 & 17.2 & 5.5\end{array}$

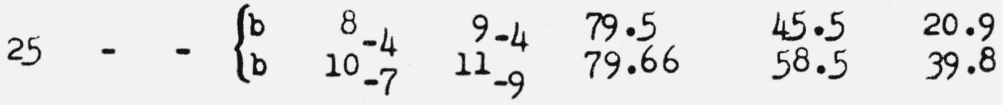

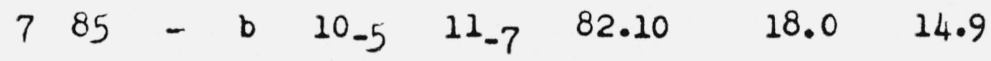

$544=$

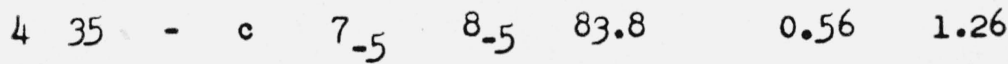

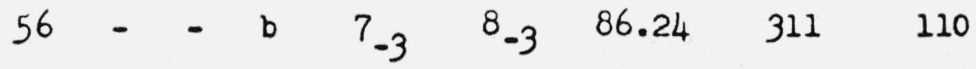

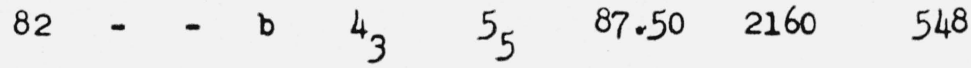

4 - $\begin{array}{lllllll}- & \mathrm{b} & 5_{-3} & 5_{3} & 90.46 & 22.7 & 4.9\end{array}$

$12-\quad\left\{\begin{array}{llllrl}b & 11-10 & 11-8 & 90.78 & 4.5 & 3.1 \\ b & 11-11 & 11-9 & 91.07 & 13.4 & 9.3\end{array}\right.$

$88-\quad\left\{\begin{array}{llllrr}\mathrm{b} & 9 & 10 & 94.46 & 159 & 77 \\ \mathrm{~b} & 4^{-7} & 5_{3}^{-7} & 94.55 & 2305 & 500 \\ \mathrm{~b} & 6_{-2}^{1} & 7_{-2} & 94.58 & 233 & 65\end{array}\right.$

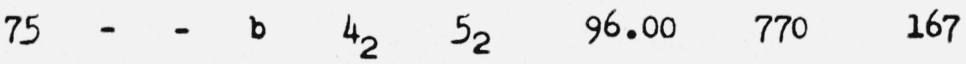

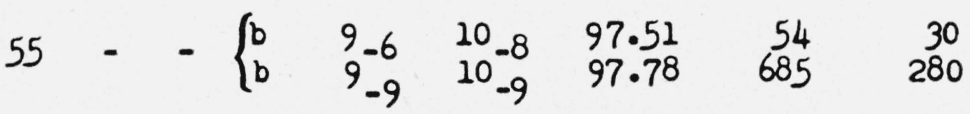

$82--\left\{\begin{array}{llllll}b & 4-1 & 51 & 99.15 & 2170 & 414 \\ c & 7-7 & 8-7 & 00.8 & 1.9 & 3.6\end{array}\right.$

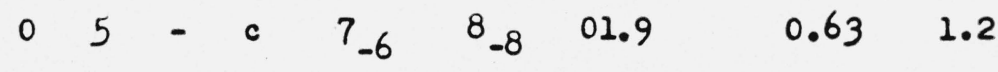

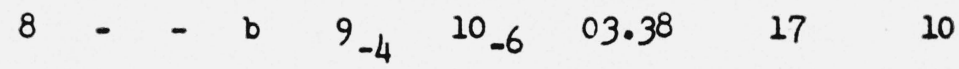

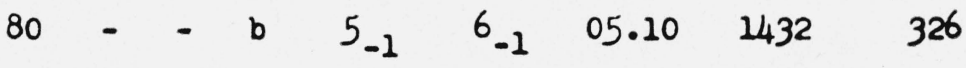

122 - 
TABLE 1-Continued.

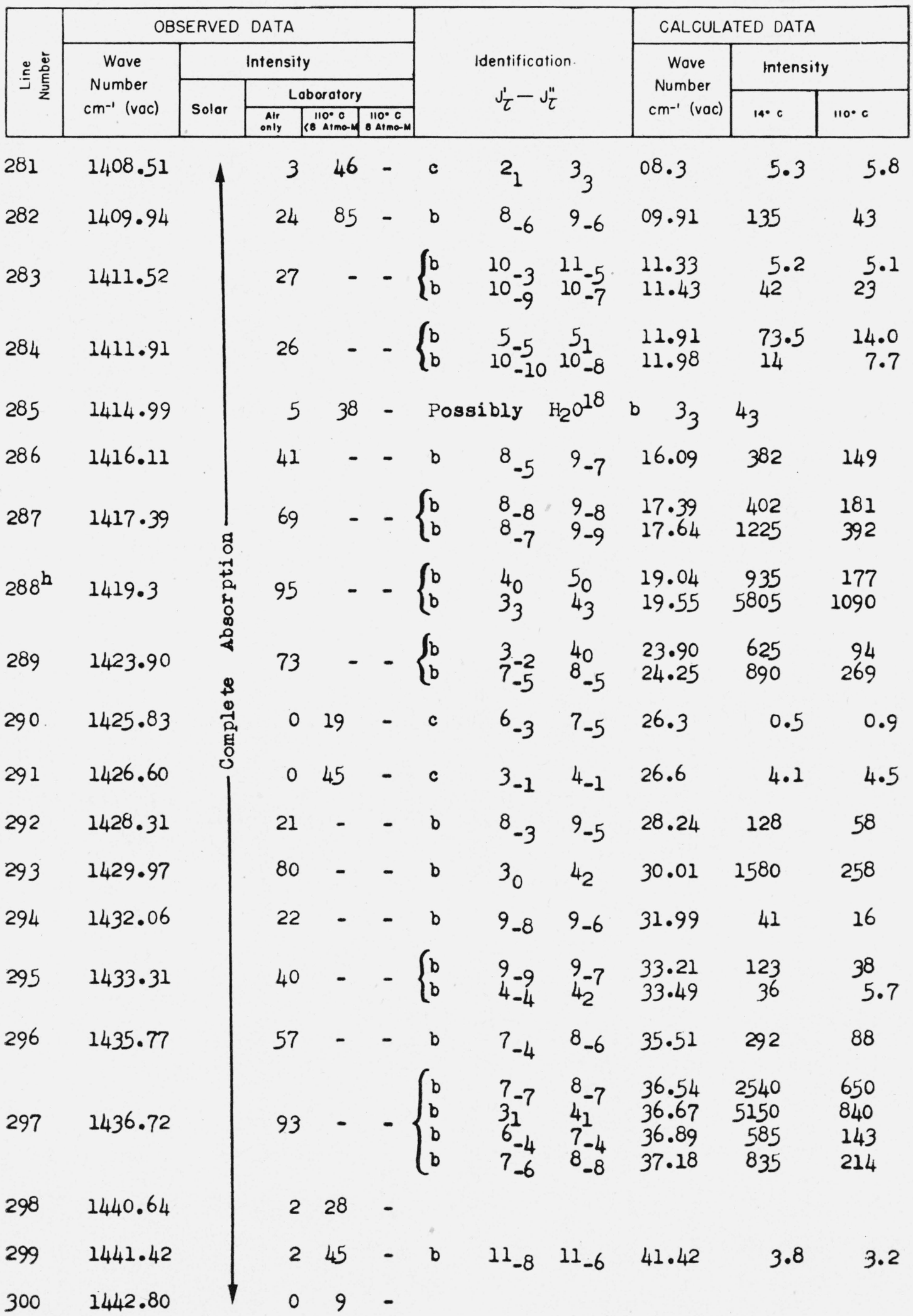


TABLE I-Continued.

\begin{tabular}{|c|c|c|c|c|c|c|c|c|c|c|c|}
\hline \multirow{3}{*}{ 导 } & \multicolumn{5}{|c|}{ OBSERVED DATA } & & & & \multicolumn{3}{|c|}{ CALCULATED DATA } \\
\hline & \multirow{2}{*}{$\begin{array}{c}\text { Wave } \\
\text { Number } \\
\mathrm{cm}^{-1} \text { (vac) }\end{array}$} & \multicolumn{4}{|c|}{ Intensity } & \multirow{2}{*}{\multicolumn{3}{|c|}{$\begin{array}{l}\text { Identification } \\
J_{\tau}^{\prime}-J_{\tau}^{\prime \prime}\end{array}$}} & \multirow{2}{*}{$\begin{array}{c}\text { Wave } \\
\text { Number } \\
\mathrm{cm}^{-1} \text { (vac) }\end{array}$} & \multicolumn{2}{|c|}{ Intensity } \\
\hline & & Solar & \multicolumn{3}{|c|}{ Laboratory } & & & & & $14^{\cdot c} c$ & $110 \cdot c$ \\
\hline 301 & 1445.09 & 4 & 7 & 60 & - & $\begin{array}{l}b \\
b\end{array}$ & $\begin{array}{r}11-8 \\
9-2\end{array}$ & $\begin{array}{l}11_{-7} \\
10_{-4}\end{array}$ & $\begin{array}{l}45.05 \\
45.12\end{array}$ & $\begin{array}{r}11.8 \\
4.9\end{array}$ & $\begin{array}{l}9.8 \\
3.4\end{array}$ \\
\hline 302 & 1446.49 & & 13 & 65 & - & $\mathrm{b}$ & $3-3$ & $3_{3}$ & 46.52 & 60 & 8.2 \\
\hline 303 & 1447.91 & & 85 & - & - & $\mathrm{b}$ & $5-3$ & $6-3$ & 47.88 & 2955 & 594 \\
\hline 304 & 1450.53 & & 1 & 19 & - & Pos & 8 sibly & $\mathrm{H}_{2} \mathrm{O}^{18}$ & b $\quad 6-5$ & $7^{7}-7$ & \\
\hline 305 & 1452.01 & & 58 & - & - & $\mathrm{b}$ & ${ }^{8}-7$ & ${ }^{8}-5$ & 52.20 & 324 & 98 \\
\hline 306 & 1453.4 & & 1 & - & - & & & & & & \\
\hline 307 & 1454.59 & & 44 & - & - & $\mathrm{b}$ & $8-8$ & ${ }^{8}-\epsilon$ & 54.64 & 109 & 33 \\
\hline 308 & 1455.26 & I & 75 & - & - & $b$ & $6-6$ & $7-6$ & 55.30 & 1600 & 310 \\
\hline 309 & 1456.49 & $\begin{array}{l}0 \\
0 \\
0 \\
0 \\
0 \\
0\end{array}$ & 95 & - & - & $\mathrm{b}$ & $6-5$ & $7-7$ & 56.45 & 4785 & 995 \\
\hline 310 & 1457.09 & $\begin{array}{l}0 \\
\text { in } \\
\text { o } \\
\text { \& }\end{array}$ & 89 & - & - & $\begin{array}{l}b \\
b\end{array}$ & $\begin{array}{l}2_{1} \\
6_{-3}\end{array}$ & $\begin{array}{l}3_{3} \\
7_{-5}\end{array}$ & $\begin{array}{l}57.11 \\
57.11\end{array}$ & $\begin{array}{l}8400 \\
1650\end{array}$ & $\begin{array}{r}1210 \\
402\end{array}$ \\
\hline 311 & 1458.24 & $\begin{array}{l}0 \\
+ \\
0 \\
0 \\
0\end{array}$ & 89 & - & - & $\mathrm{b}$ & $z_{2}$ & $3_{2}$ & 58.39 & 2880 & 413 \\
\hline 312 & 1459.26 & $\begin{array}{l}\text { Oे } \\
\text { E् } \\
\text { ટ) }\end{array}$ & 84 & - & - & $\mathrm{b}$ & $4-2$ & $5-2$ & 59.30 & 1525 & 260 \\
\hline 313 & 1460.76 & 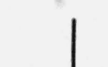 & 2 & 68 & - & & & & & & \\
\hline 314 & 1462.71 & & 2 & - & & & & & & & \\
\hline 315 & 1464.92 & & 92 & - & - & $\mathrm{b}$ & $2-1$ & 31 & 64.91 & 4800 & 635 \\
\hline 316 & 1466.59 & & 4 & - & - & Pos & sibly & $\mathrm{H}_{2} \mathrm{O}^{18}$ & b $\quad 3-1$ & $4^{4}-1$ & \\
\hline 317 & 1467.61 & & 6 & - & - & Pos & sibly & $\mathrm{H}_{2} \mathrm{O}^{18}$ & $5-5$ & $6-5$ & \\
\hline 318 & 1459.3 & 5 & 2 & - & - & & & & & & \\
\hline 319 & 1472.0 & & 98 & - & . & & $\begin{array}{l}7-6 \\
3-1\end{array}$ & $\begin{array}{l}7-4 \\
4^{-1}\end{array}$ & $\begin{array}{l}71.77 \\
71.92\end{array}$ & $\begin{array}{r}441 \\
6580\end{array}$ & $\begin{array}{l}107 \\
960\end{array}$ \\
\hline 20 & 1473.44 & & 94 & - & - & $\mathrm{b}$ & ${ }^{5}-5$ & $6_{-5}$ & 73.46 & 7980 & 1410 \\
\hline
\end{tabular}


TABLE I-Continued.

\begin{tabular}{|c|c|c|c|c|c|}
\hline \multirow{4}{*}{ 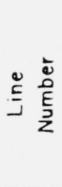 } & \multicolumn{5}{|c|}{ OBSERVED DATA } \\
\hline & \multirow{3}{*}{$\begin{array}{c}\text { Wave } \\
\text { Number } \\
\mathrm{cm}^{-1} \text { (vac) }\end{array}$} & \multicolumn{4}{|c|}{ Intensity } \\
\hline & & \multirow{2}{*}{ Solar } & \multicolumn{3}{|c|}{ Laboratory } \\
\hline & & & $\begin{array}{l}\text { Alr } \\
\text { only }\end{array}$ & 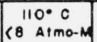 & $\begin{array}{l}110^{\circ} \mathrm{C} \\
8 \Delta t \operatorname{mo}-\mathrm{M}\end{array}$ \\
\hline
\end{tabular}

Identification
$J_{\tau}^{\prime}-J_{\tau}^{\prime \prime}$

\begin{tabular}{|c|c|c|}
\hline \multicolumn{2}{|c|}{ CALCULATED DATA } \\
\hline $\begin{array}{c}\text { Wave } \\
\text { Number } \\
\mathrm{cm}^{-1} \text { (vac) }\end{array}$ & \multicolumn{2}{|c|}{ Intensity } \\
\cline { 2 - 3 } & $14 . \mathrm{c}$ & $110 . \mathrm{c}$ \\
\hline
\end{tabular}

3211476.29 $\begin{array}{ll}322 & 1478.1 \\ 323 & 1480.4\end{array}$ $\begin{array}{ll}322 & 1478.1 \\ 323 & 1480.4\end{array}$

$324 \quad 1481.33$

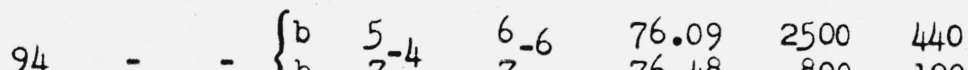

$325 \quad 1482.5$

$326 \quad 1484.3$

$327 \quad 1486.27$

$328 \quad 1487 \cdot 34$

$329 \quad 1489.23$

$330 \quad 1489.81$

$331 \quad 1490.81$

3321496.23

140 -

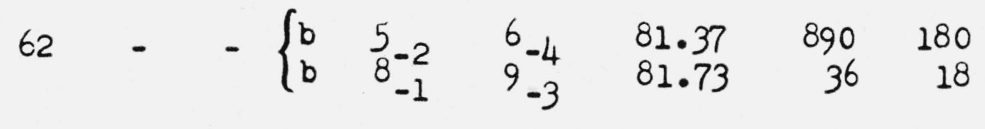

$0 \quad 18$

- b $10_{-1} \quad 11-3$

84.44

$1.5 \quad 1.7$

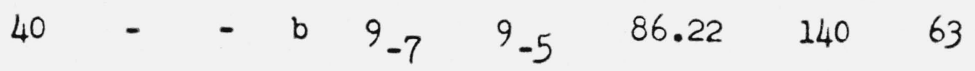

91 - $\quad \begin{array}{llllll} & \mathrm{b} & 2_{0} & 30 & (87.34) & 2970 \quad 390\end{array}$

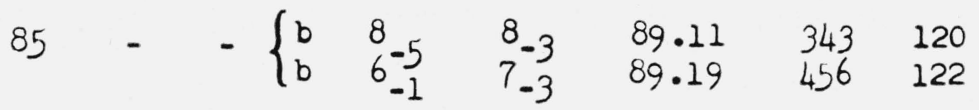

88 - $\quad \begin{array}{llllll}b^{2} & b_{-5} & 6_{-3} & 89.81 & 1685 & 340\end{array}$

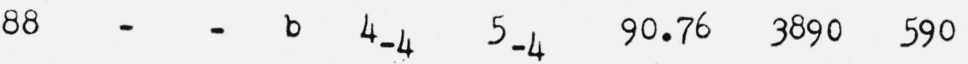

94 - - b $4-3 \quad 5-5 \quad 96.27 \quad 11560 \quad 1760$

$333 \quad 1498.79$

$92-\quad\left\{\begin{array}{llllll}b & 1 & 2 & (98.79) & 3145 & 390 \\ b & 6 & 6_{-6}^{2} & 98.86 & 610 & 120\end{array}\right.$

$334 \quad 1500.51$

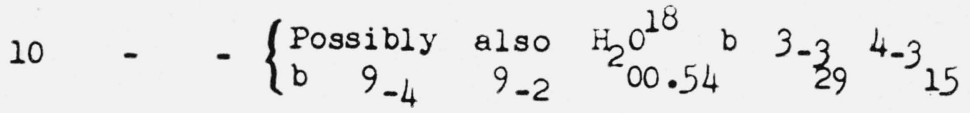

$335 \quad 1501.83$

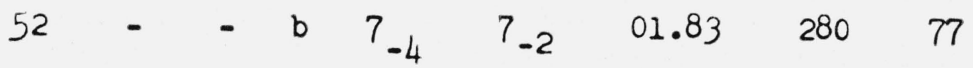

$336^{j} 1506$

$99-\quad-\left\{\begin{array}{llllrr}b & 1_{1} & 2_{1} & 05.57 & 11460 & 1370 \\ b & 5-4 & 5-2 & 06.68 & 1065 & 180 \\ b & 3-3 & 4-3 & 07.09 & 14670 & 1960\end{array}\right.$

$337 \quad 1508.55$

97 - $\quad$ - b $\quad 4_{-1} \quad 5_{-3} \quad 08.49 \quad 3340 \quad 560$

$85-\quad-\left\{\begin{array}{llllll}b & 8 & 8 & 09.56 & 111 & 54 \\ b & 6)^{-1} & 63 & 09.72 & 365 & 111 \\ b & 53 & 55 & 09.73 & 540 & 137\end{array}\right.$

$339 \quad 1510.56$

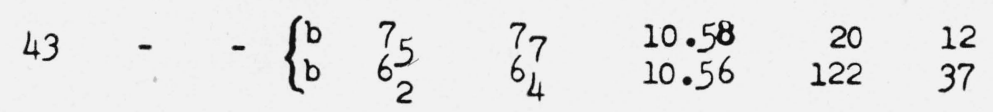


TABLE I-Continued.

\begin{tabular}{|c|c|c|c|c|c|c|c|c|c|c|c|}
\hline \multirow{4}{*}{ 冚总 } & \multicolumn{5}{|c|}{ oBSERVED DATA } & & & & \multicolumn{3}{|c|}{ CALCULATED DATA } \\
\hline & \multirow{3}{*}{$\begin{array}{l}\text { Wave } \\
\text { Number } \\
\mathrm{cm}^{-1} \text { (vac) }\end{array}$} & \multicolumn{4}{|c|}{ Intensity } & & \multirow{3}{*}{\multicolumn{2}{|c|}{$\begin{array}{c}\text { Identification } \\
J_{\tau}^{\prime}-J_{\tau}^{\prime \prime}\end{array}$}} & \multirow{3}{*}{$\begin{array}{c}\text { Wave } \\
\text { Number } \\
\mathrm{cm}^{-1} \text { (vac) }\end{array}$} & \multicolumn{2}{|c|}{ Intensitỹ } \\
\hline & & \multirow{2}{*}{ Solar } & \multicolumn{3}{|c|}{ Labor.atory } & & & & & $10^{\circ} \mathrm{c}$ & \\
\hline & & & $\begin{array}{c}\text { Air } \\
\text { only } \\
\end{array}$ & 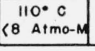 & 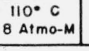 & & & & & $144^{\circ} \mathrm{C}$ & $110^{\circ} \mathrm{C}$ \\
\hline 340 & 1512.37 & 4 & 90 & - & - & $b$ & $\begin{array}{l}7 \\
7^{1} \\
6_{-3}^{-2}\end{array}$ & $\begin{array}{l}7_{3} \\
7_{0}^{0} \\
6_{-1}^{6}\end{array}$ & $\begin{array}{l}12.22 \\
12.30 \\
12.34\end{array}$ & $\begin{array}{r}159 \\
185 \\
1735\end{array}$ & $\begin{array}{r}60 \\
59 \\
396\end{array}$ \\
\hline 341 & 1515.01 & & 72 & - & - & $b$ & ${ }^{6}-1$ & $6_{1}$ & 14.84 & 980 & 250 \\
\hline 342 & 1516.77 & & 94 & - & - & $\mathrm{b}$ & $\begin{array}{l}5 \\
4_{1}^{0}\end{array}$ & $\begin{array}{l}5_{3}^{2} \\
3\end{array}$ & $\begin{array}{l}16.32 \\
16.73\end{array}$ & $\begin{array}{r}475 \\
1420\end{array}$ & $\begin{array}{l}103 \\
267\end{array}$ \\
\hline 343 & 1517.50 & 동 & 96 & - & - & b & $\begin{array}{l}3-2 \\
4_{2}^{-2}\end{array}$ & $\frac{4}{4_{4}^{-4}}$ & $\begin{array}{l}17.59 \\
17.95\end{array}$ & $\begin{array}{r}4680 \\
470\end{array}$ & $\begin{array}{r}620 \\
88\end{array}$ \\
\hline 344 & 1520.36 & $\begin{array}{l}0 \\
0 \\
0 \\
0 \\
0 \\
0 \\
0\end{array}$ & 93 & - & - & $\begin{array}{l}b \\
b\end{array}$ & $\begin{array}{l}5 \\
5_{-2}^{1}\end{array}$ & $\begin{array}{l}5_{3} \\
5_{0}\end{array}$ & $\begin{array}{l}20.18 \\
20.24\end{array}$ & $\begin{array}{l}1470 \\
1330\end{array}$ & $\begin{array}{l}320 \\
253\end{array}$ \\
\hline 345 & 1521.4 & $\stackrel{\infty}{+}$ & 95 & - & - & $\begin{array}{l}b \\
b\end{array}$ & $\begin{array}{l}4 \\
5^{-3} \\
-5\end{array}$ & $\begin{array}{l}4-1 \\
5-3\end{array}$ & $\begin{array}{l}21.25 \\
21.25\end{array}$ & $\begin{array}{l}5230 \\
3870\end{array}$ & $\begin{array}{l}760 \\
650\end{array}$ \\
\hline 346 & 1522.67 & - & 92 & - & - & $b$ & ${ }^{2}-2$ & $3-2$ & 22.66 & 5300 & 650 \\
\hline 347 & 1525.1 & () & 72 & - & - & $\mathrm{b}$ & ${ }^{6} 0$ & $6_{2}$ & 24.86 & 347 & 88 \\
\hline 348 & 1525.52 & & 92 & - & - & $b$ & ${ }^{4}-1$ & 4 & 25.45 & 4100 & 670 \\
\hline 349 & 1527.38 & & 79 & - & - & $\mathrm{b}$ & ${ }^{7}-5$ & ${ }^{7}-3$ & 27.48 & 1254 & 335 \\
\hline 350 & 1528.66 & & 75 & - & - & $\mathrm{b}$ & $3_{0}$ & $3_{2}$ & 28.62 & 1240 & 179 \\
\hline 351 & 1531.71 & † & 55 & - & - & $\mathrm{b}$ & ${ }^{7}-1$ & ${ }^{7} 1$ & 31.64 & 595 & 185 \\
\hline
\end{tabular}

Note: Between lines 351 and 352 the absorption has not been measured, since our resolution is not improved over that published[3]. The calculated spectrum from our energy levels yields results agreeirg to $\pm 0.2 \mathrm{~cm}^{-1}$ with the earlier work. 
TABLE I-Continued.

\begin{tabular}{|c|c|c|c|c|c|c|c|c|c|c|c|}
\hline \multirow{3}{*}{ 范 } & \multicolumn{5}{|c|}{ OBSERVED DATA } & & & & \multicolumn{3}{|c|}{ CALCULATED DATA } \\
\hline & \multirow{2}{*}{$\begin{array}{c}\text { Wove } \\
\text { Number } \\
\mathrm{cm}^{-1} \text { (vac) }\end{array}$} & \multicolumn{4}{|c|}{ Intensity } & \multirow{2}{*}{\multicolumn{3}{|c|}{$\begin{array}{l}\text { Identification } \\
J_{\tau}^{\prime}-J_{\tau}^{\prime \prime}\end{array}$}} & \multirow{2}{*}{$\begin{array}{l}\text { Wove } \\
\text { Number } \\
\mathrm{cm}^{-1} \text { (vac }\end{array}$} & \multicolumn{2}{|c|}{ Intensity } \\
\hline & & Solar & \multicolumn{3}{|c|}{ Laboratory } & & & & & vac) & $110 . \mathrm{c}$ \\
\hline 352 & 1761.88 & 4 & 88 & - & - & b & $6-3$ & $5-3$ & 61.86 & 6388 & 1070 \\
\hline 353 & 1764.22 & & 7 & - & - & $\mathrm{P}$ & ssibly & $\mathrm{H}_{2} \mathrm{O}^{18}$ & b & $3_{3}$ & 21 \\
\hline 354 & 1765.40 & & 6 & - & - & b & $10_{-6}$ & 10 -8 & 65.38 & 29 & 16 \\
\hline 355 & 1767.3 & & 20 & - & - & b & $9_{-2}$ & $9-4$ & 67.06 & 54 & 25 \\
\hline 356 & 1768.20 & & 87 & - & - & $\left\{\begin{array}{l}b \\
b \\
b\end{array}\right.$ & $\begin{array}{l}9-7 \\
9-9 \\
9-8\end{array}$ & $\begin{array}{l}9-9 \\
8-7 \\
8-8\end{array}$ & $\begin{array}{l}68.06 \\
68.23 \\
68.41\end{array}$ & $\begin{array}{r}275 \\
2910 \\
970\end{array}$ & $\begin{array}{r}88 \\
740 \\
250\end{array}$ \\
\hline 357 & 1771.38 & & 91 & - & - & $b$ & $3_{2}$ & 22 & 71.45 & 6050 & 725 \\
\hline 358 & 1772.64 & & 93 & - & - & b & $3_{3}$ & 21 & 72.80 & 17850 & 2140 \\
\hline 359 & 1775.64 & $\underset{-7}{5}$ & 77 & - & - & b & $7-4$ & $6-4$ & 75.61 & 1320 & 260 \\
\hline 360 & 1778.6 & 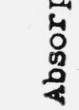 & 22 & - & - & $\left\{\begin{array}{l}b \\
c\end{array}\right.$ & $4_{1}^{10}$ & $3_{1}^{-5}$ & $\begin{array}{l}78.57 \\
78.64\end{array}$ & $\begin{array}{r}55 \\
8\end{array}$ & $\begin{array}{r}33 \\
8\end{array}$ \\
\hline 361 & 1779.14 & $\stackrel{9}{9}$ & 49 & - & - & $\left\{\begin{array}{l}\mathrm{b} \\
\mathrm{b}\end{array}\right.$ & $\begin{array}{r}8 \\
10^{-4}-5\end{array}$ & $\begin{array}{r}7-2 \\
10-7\end{array}$ & $\begin{array}{l}79.2 \\
79.15\end{array}$ & $\begin{array}{r}210 \\
84\end{array}$ & $\begin{array}{l}59 \\
46\end{array}$ \\
\hline 362 & 1780.70 & : & 84 & - & - & $\left\{\begin{array}{l}b \\
b\end{array}\right.$ & $\begin{array}{l}40 \\
8-6\end{array}$ & $\begin{array}{l}3-2 \\
7-4\end{array}$ & $\begin{array}{l}80.77 \\
80.92\end{array}$ & $\begin{array}{r}1485 \\
715\end{array}$ & $\begin{array}{l}181 \\
174\end{array}$ \\
\hline 363 & 1781.96 & & 28 & - & - & b & $9-3$ & $8_{-1}$ & 82.04 & 80 & 33 \\
\hline 364 & 1783.95 & & 23 & - & - & b & $4_{2}$ & $4-4$ & 84.08 & 80 & 11 \\
\hline 365 & 1784.93 & & 76 & - & - & $\mathrm{b}$ & $10-9$ & $9-9$ & 85.03 & 1810 & 580 \\
\hline 366 & 1788.45 & & 18 & - & - & $\left\{\begin{array}{l}b \\
b\end{array}\right.$ & $\begin{array}{c}82 \\
10_{-8}^{2}\end{array}$ & ${ }_{10}^{8}{ }^{8}-10$ & $\begin{array}{l}88.38 \\
88.79\end{array}$ & $\begin{array}{l}73 \\
34\end{array}$ & $\begin{array}{l}30 \\
14\end{array}$ \\
\hline 367 & 1790.1 & & 37 & - & - & $\left\{\begin{array}{l}b \\
b\end{array}\right.$ & $\begin{array}{l}10-7 \\
11-7\end{array}$ & $\begin{array}{l}10-9 \\
11-9\end{array}$ & $\begin{array}{l}90.09 \\
90.0\end{array}$ & $\begin{array}{r}104 \\
27\end{array}$ & $\begin{array}{l}42 \\
18\end{array}$ \\
\hline 368 & 1791.02 & & 88 & - & - & b & $8_{-5}$ & $7-5$ & 91.05 & 2210 & 540 \\
\hline 369 & 1792.63 & & 92 & - & - & b & $4_{1}$ & 31 & 92.73 & 12050 & 1590 \\
\hline 370 & 1795.22 & $\downarrow$ & 41 & - & - & b & 6 & $6_{2}$ & 95.28 & 235 & 60 \\
\hline
\end{tabular}


TABLE I-Continued.

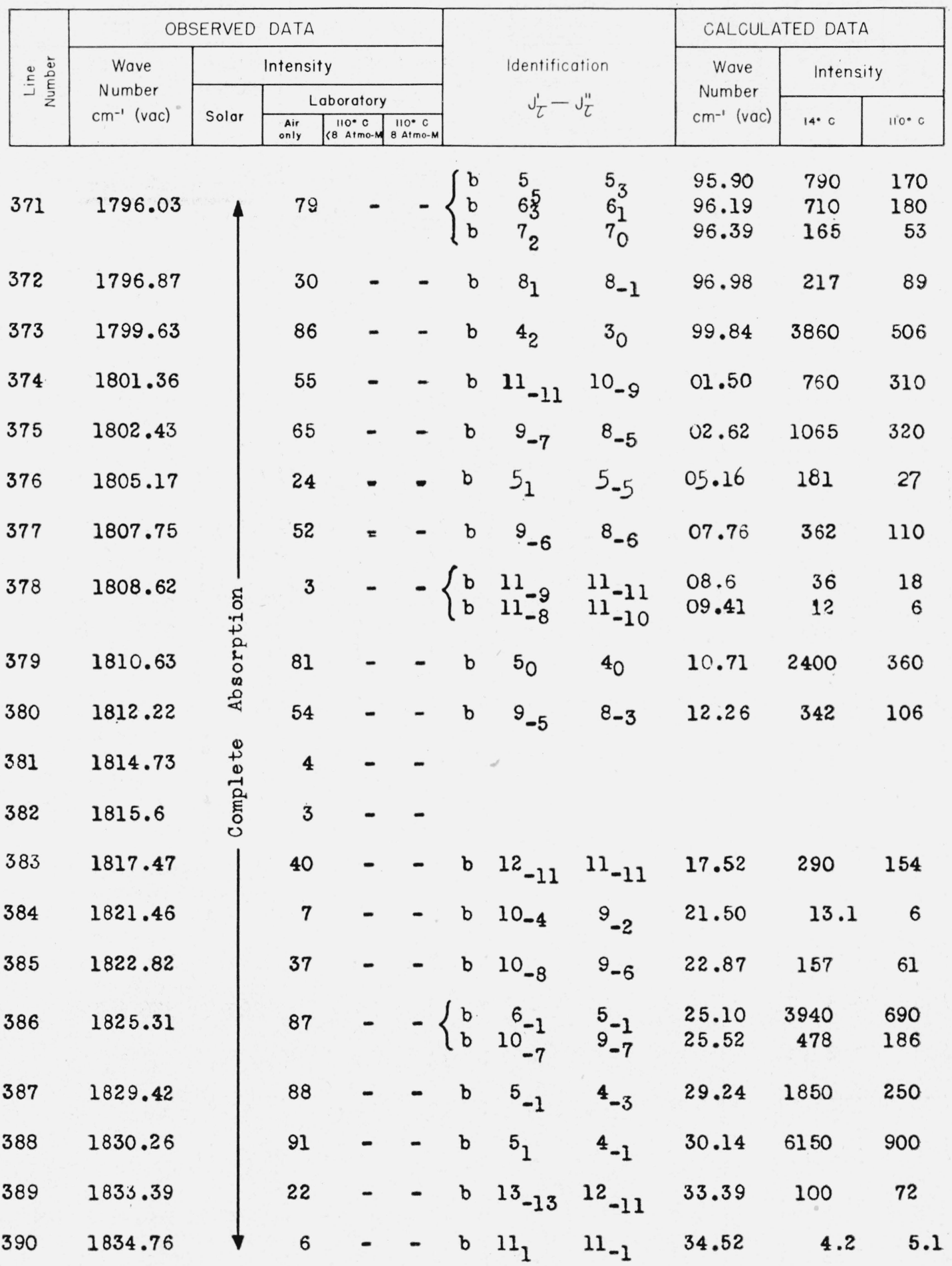


TABLE I-Continued.

\begin{tabular}{|c|c|c|c|c|c|c|c|c|c|}
\hline \multirow{4}{*}{ 站总 } & \multicolumn{5}{|c|}{ OBSERVED DATA } & \multirow{4}{*}{$\begin{array}{l}\text { Identification } \\
J_{\tau}^{\prime}-J_{\tau}^{\prime \prime}\end{array}$} & \multicolumn{3}{|c|}{ CALCULATED DATA } \\
\hline & \multirow{3}{*}{$\begin{array}{c}\text { Wave } \\
\text { Number } \\
\mathrm{cm}^{-1} \text { (voc) }\end{array}$} & \multicolumn{4}{|c|}{ Intensity } & & \multirow{3}{*}{$\begin{array}{c}\text { Wave } \\
\text { Number } \\
\mathrm{cm}^{-1} \text { (vac) }\end{array}$} & \multicolumn{2}{|c|}{ Intensity } \\
\hline & & \multirow{2}{*}{ Solar } & \multicolumn{3}{|c|}{ Laboratory } & & & & \\
\hline & & & Alr & 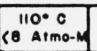 & 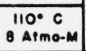 & & & $14^{\circ} \mathrm{c}$ & $1100 \mathrm{c}$ \\
\hline
\end{tabular}

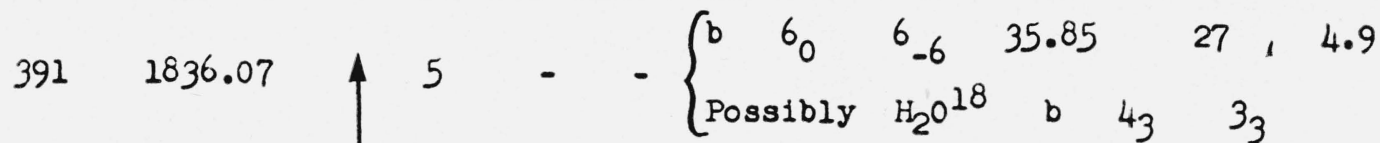

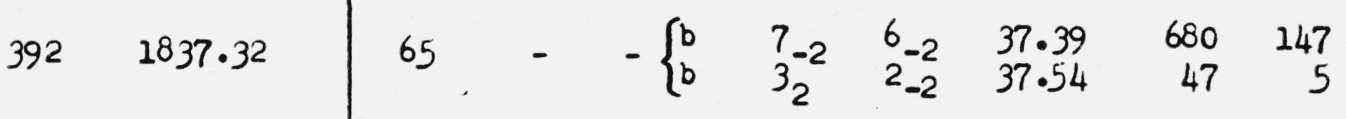

$\begin{array}{lllllllllll}393 & 1839.29 & 4 & 20 & - & c & 4_{3} & 3_{3} & 39.1 & 11 & 12\end{array}$

$394 \quad 1840.5$

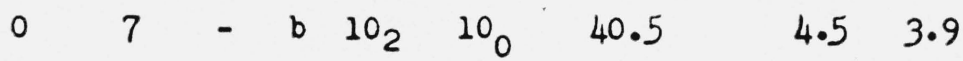

$395 \quad 1842.25$

$37-\quad \begin{array}{rrrrrr}b & 11-9 & 10-7 & 42.10 & 187 & 103 \\ b & 10-6 & 9-4 & 42.23 & 52 & 25\end{array}$

$396 \quad 1844.24$

$92-\quad-\left\{\begin{array}{lccrrr}b & 11-8 & 10-8 & 43.44 & 62 & 34 \\ b & 43 & 33 & 44.20 & 16000 & 2300\end{array}\right.$

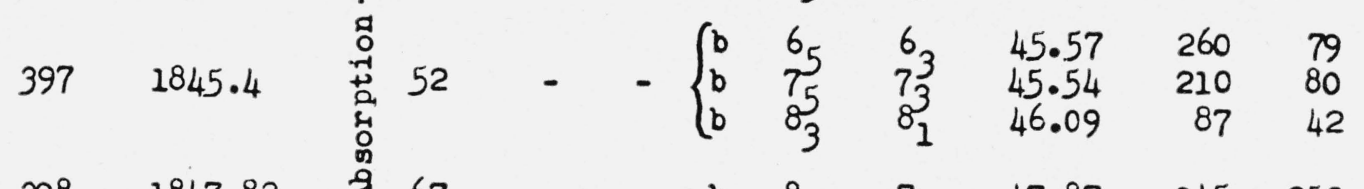

$398 \quad 1847.82$ \& $67 \quad-\quad \begin{array}{lllllll} & - & 8_{-3} & 7_{-3} & 47.87 & 945 & 253\end{array}$

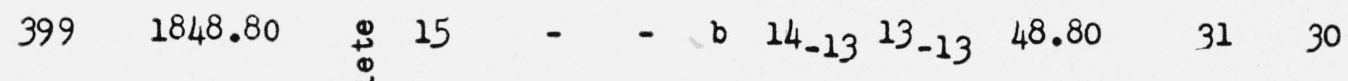

$\begin{array}{llllllllllllll}400 & 1852.44 & \overrightarrow{0} & 8 & 19 & - & b & 5 & 5 & 5 & 52.38 & 64 & 9.5\end{array}$

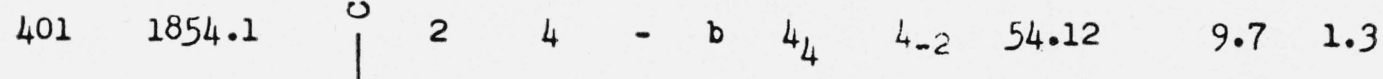

\begin{tabular}{ll|lllllllll}
402 & 1856.30 & 4 & 11 & - & $b$ & $6_{2}$ & $6-4$ & 56.40 & 28.4 & 5.7
\end{tabular}

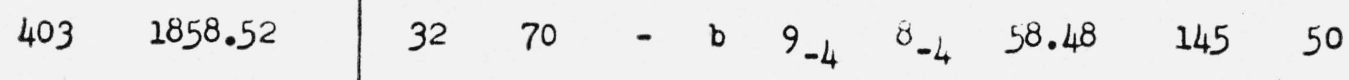

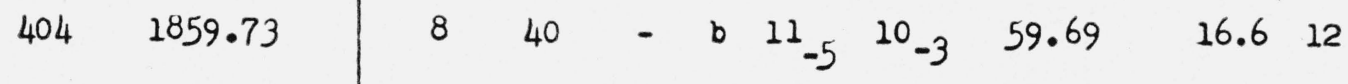

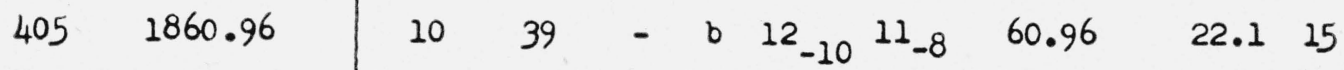

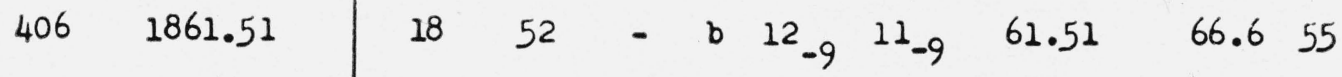

\begin{tabular}{ll|lllllllll}
407 & 1863.1 & 0 & 4 & - & $c$ & $5_{3}$ & 41 & 63.6 & 4.5 & 5.7
\end{tabular}

$\begin{array}{lllllllllll}408 & 1864.07 & 7 & 34 & - & b & 15 & -15^{14}-13 & 64.06 & 8.8 & 12\end{array}$

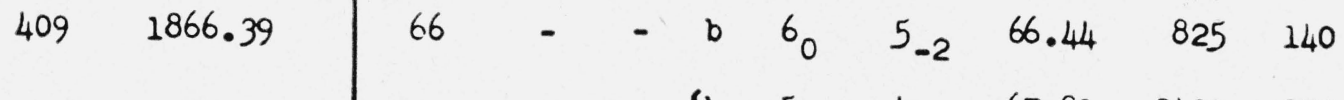

$410 \quad 1867.94 \downarrow 82--\left\{\begin{array}{llllrr}b & 52 & 42 & 67.81 & 2400 & 390 \\ b & 41 & 3 & 68.12 & 388 & 47\end{array}\right.$ 
TABLE 1-Continued.

\begin{tabular}{|c|c|c|c|c|c|c|c|c|c|}
\hline \multirow{4}{*}{ 站 } & \multicolumn{5}{|c|}{ OBSERVED DATA } & \multirow{4}{*}{$\begin{array}{l}\text { Identification } \\
J_{\tau}^{\prime}-J_{\tau}^{\prime \prime}\end{array}$} & \multicolumn{3}{|c|}{ CALCULATED DATA } \\
\hline & \multirow{3}{*}{$\begin{array}{c}\text { Wave } \\
\text { Number } \\
\mathrm{cm}^{-1} \text { (vac) }\end{array}$} & \multicolumn{4}{|c|}{ Intensity } & & \multirow{3}{*}{$\begin{array}{c}\text { Wave } \\
\text { Number } \\
\mathrm{cm}^{-1} \text { (vac) }\end{array}$} & \multicolumn{2}{|c|}{ Intensity } \\
\hline & & \multirow{2}{*}{ Solar } & \multicolumn{3}{|c|}{ Laboratory } & & & & \\
\hline & & & $\begin{array}{c}\text { Air } \\
\text { only }\end{array}$ & 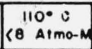 & $\begin{array}{l}110^{\circ} \mathrm{C} \\
8 \text { Aimo-M }\end{array}$ & & & $14^{\circ} \mathrm{C}$ & $110^{\circ} \mathrm{c}$ \\
\hline
\end{tabular}

\begin{tabular}{|c|c|c|c|c|c|c|c|c|c|c|}
\hline 1869.25 & 4 & 90 & - & - & $\mathrm{b}$ & $5_{3}$ & $4_{1}$ & 69.34 & 7220 & 1180 \\
\hline 1870.78 & & 45 & - & - & b & $10=5$ & $9_{-5}$ & 70.91 & 179 & 80 \\
\hline $1876 . .67$ & 1 & 5 & 11 & - & $\mathrm{b}$ & $7-1$ & $7-7$ & 76.59 & 25.0 & 5.2 \\
\hline 1879.43 & 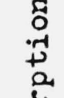 & 12 & 50 & - & $\mathrm{b}$ & ${ }^{13}-11$ & $12-9$ & 79.40 & 28.2 & 26 \\
\hline 1882.3 & $\begin{array}{l}0 \\
0 \\
0 \\
0\end{array}$ & 0 & 2 & - & & & & & & \\
\hline 1884.63 & & 36 & 62 & - & $\mathrm{b}$ & $6_{-2}$ & $5_{-4}$ & 84.64 & 243 & 37 \\
\hline 1885.28 & $\begin{array}{l}+ \\
\stackrel{J}{0} \\
\stackrel{2}{0}\end{array}$ & 9 & 42 & - & $\mathrm{b}$ & ${ }^{11}-6$ & $10_{-6}$ & 85.36 & 22 & 13 \\
\hline 1889.59 & E & 87 & 95 & - & $\mathrm{b}$ & ${ }^{6} 1$ & ${ }^{5} 1$ & 89.60 & 3715 & 709 \\
\hline 1892.63 & & 2 & 17 & - & $b$ & $12-8$ & ${ }^{11}-6$ & $(92.63)$ & 7.1 & 5.8 \\
\hline 1893.87 & & 7 & 25 & - & $\mathrm{b}$ & $7_{7}$ & $7_{5}$ & 93.63 & 52 & 23 \\
\hline 1895.27 & $\nabla$ & 65 & 85 & - & $\begin{array}{l}b \\
b\end{array}$ & $\begin{array}{l}8_{5} \\
6 \\
9_{5}^{2}\end{array}$ & $\begin{array}{l}8_{3} \\
5_{0}^{3} \\
9 \\
9_{3}\end{array}$ & $\begin{array}{l}94.99 \\
95.27 \\
95.19\end{array}$ & $\begin{array}{r}37 \\
1230 \\
18\end{array}$ & $\begin{array}{r}21 \\
230 \\
14\end{array}$ \\
\hline
\end{tabular}

\begin{tabular}{|c|c|c|c|c|c|c|c|c|c|c|c|}
\hline 422 & 1897.57 & 40 & 5 & 32 & - & b & $14-11$ & $3-11$ & $(97.57)$ & 8.1 & 10.4 \\
\hline 423 & 1898.81 & 10 & 0 & 6 & - & & & & & & \\
\hline 424 & 1901.87 & 75 & 10 & 40 & - & $\mathrm{b}$ & $12-7$ & $1_{-7}$ & 01.86 & 22 & 18 \\
\hline 425 & 1904.50 & 99 & 23 & 43 & - & $b$ & $5_{0}$ & $4_{-4}$ & 04.40 & 159 & 21 \\
\hline 426 & 1908.12 & & 53 & 82 & - & $\mathrm{b}$ & ${ }^{7} \dot{0}$ & $6_{0}$ & 08.12 & 555 & 128 \\
\hline 427 & 1910.09 & & 68 & 87 & - & $\mathrm{b}$ & ${ }^{7}-1$ & $6-3$ & 09.95 & 925 & 186 \\
\hline 428 & 1914.70 & 99 & 3 & 25 & - & & ssibly & $\mathrm{H}_{2} \mathrm{O}^{18}$ & b $\quad 7_{1}$ & $6-1$ & \\
\hline 429 & 1915.30 & & 1 & 15 & - & d & $6_{-5}$ & ${ }^{7}-$ & 15.16 & 0.07 & 0.10 \\
\hline 430 & 1918.08 & & 82 & - & - & 0 & $5_{5}$ & $4_{3}$ & 18.08 & 7660 & 1440 \\
\hline
\end{tabular}


TABLE I-Continued.

\begin{tabular}{|c|c|c|c|c|c|c|c|c|}
\hline \multirow{4}{*}{ 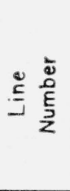 } & \multicolumn{4}{|c|}{ OBSERVED DATA } & \multirow{4}{*}{$\begin{array}{l}\text { Identification } \\
\qquad J_{\tau}^{\prime}-J_{\tau}^{\prime \prime}\end{array}$} & \multicolumn{3}{|c|}{ CALCULATED DATA } \\
\hline & \multirow{3}{*}{$\begin{array}{c}\text { Wave } \\
\text { Number } \\
\mathrm{cm}^{-1} \text { (vac) }\end{array}$} & \multicolumn{3}{|c|}{ Intensity } & & \multirow{3}{*}{$\begin{array}{c}\text { Wave } \\
\text { Number } \\
\mathrm{cm}^{-1} \text { (vac) }\end{array}$} & \multicolumn{2}{|c|}{ Intensity } \\
\hline & & \multirow{2}{*}{ Solar } & \multicolumn{2}{|c|}{ Laboratory } & & & & \\
\hline & & & $\begin{array}{l}\text { Air } \\
\text { only }\end{array}$ & 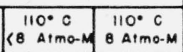 & & & $14^{\circ} \mathrm{c}$ & $110 . \mathrm{c}$ \\
\hline
\end{tabular}

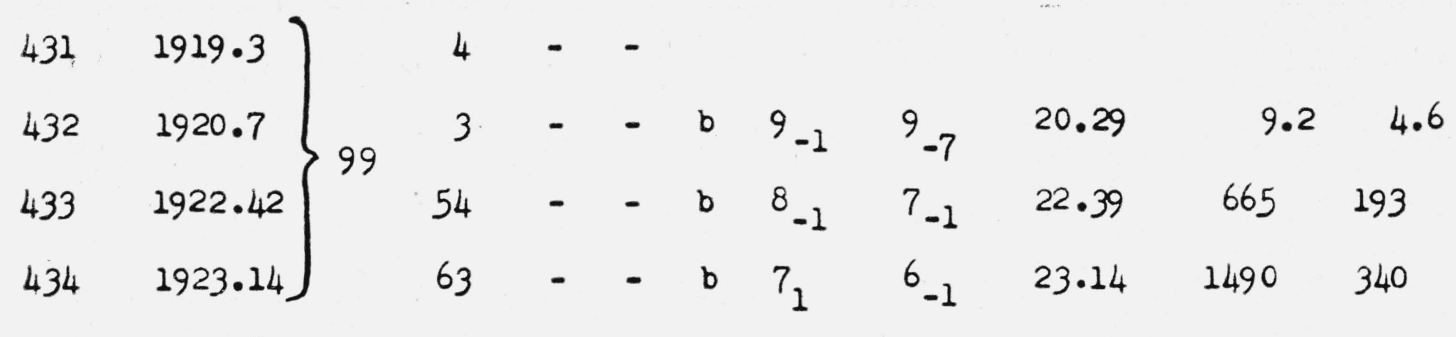

$\begin{array}{llllll}435 & 1927.82 & 30 & 3 & -\end{array}$

$\begin{array}{lllllllllllll}436 & 1933.09 & 97 & 14 & - & - & 9_{-2} & 8_{-2} & 33.23 & 86 & 32\end{array}$ $\begin{array}{llllll}437 & 1935.24 & 15 & 0 & 20 & -\end{array}$

$\begin{array}{llllllllllll}438 & 1937.87 & 40 & 0 & 40 & - & \mathrm{b} & 8_{2} & 8_{-4} & 37.17 & 2.8 & 1.1\end{array}$

$\begin{array}{lllllllllllll}439 & 1939.2 & 15 & 0 & 9 & - & b & 9_{1} & 9_{-5} & 39.51 & 4.7 & 2.1\end{array}$

$\begin{array}{ll}440 & 1940.2\end{array} \quad 3 \quad-\quad \begin{array}{lllllll}3 & 8 & 8 & 8 & 40.35 & 8.0 & 5.8\end{array}$

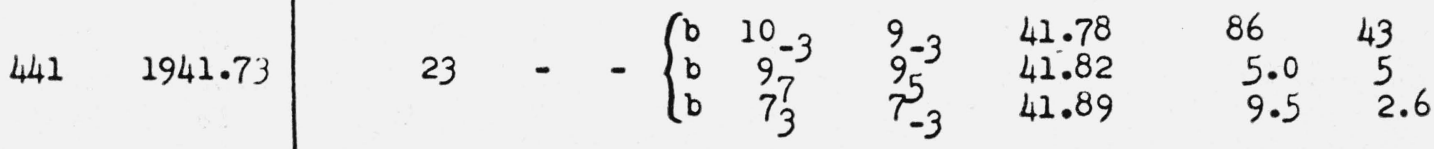

$\left.\begin{array}{ll}442 & 1942.49\end{array}\right\} 99 \quad 80-\begin{array}{lllllll}b & 6 & 5 & 42.61 & 3026 & 660 \\ b & 6 & 3 & 5 & 42.86 & 1009 & 220\end{array}$

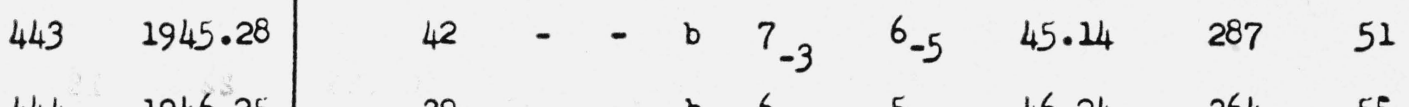

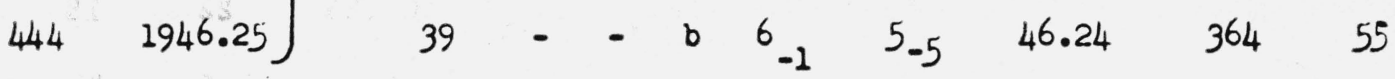

$\begin{array}{llllllllllll}445 & 1949.15 & 70 & 9 & 71 & - & b & 11 & 10_{-4} & 49.28 & 9.2 & 6.2\end{array}$

$\begin{array}{lllllllllllll}446 & 1949.96 & 10 & 0 & 10 & - & 6_{4} & 6_{-2} & 50.31 & 2.5 & 0.5\end{array}$

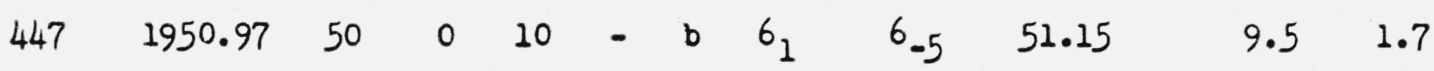

$\begin{array}{lllllllllllll}448 \quad 1954.99 & 99 & 36 & 97 & - & \mathrm{b} & 8_{0} & 7_{-2} & 55.02 & 202 & 56\end{array}$

$\begin{array}{llllllllllllll}449 & 1956.25 & 97 & 2 & 75 & - & \mathrm{b} & 4 & 3 & 3-1 & 56.24 & 28.3 & 3.5\end{array}$

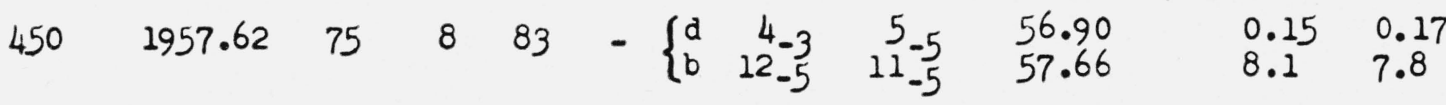


TABLE 1-Continued.

\begin{tabular}{|c|c|c|c|c|c|c|c|}
\hline \multirow{3}{*}{ 竞 } & \multicolumn{3}{|c|}{ OBSERVED DATA } & \multirow{3}{*}{$\begin{array}{c}\text { Identification } \\
J_{\tau}^{\prime}-J_{\tau}^{\prime \prime}\end{array}$} & \multicolumn{3}{|c|}{ CALCULATED DATA } \\
\hline & \multirow{2}{*}{$\begin{array}{c}\text { Wave } \\
\text { Number } \\
\mathrm{cm}^{-1} \text { (vac) }\end{array}$} & \multicolumn{2}{|c|}{ Intensity } & & \multirow{2}{*}{$\begin{array}{c}\text { Wove } \\
\text { Number } \\
\mathrm{cm}^{-1} \text { (voc) }\end{array}$} & \multicolumn{2}{|c|}{ Intensity } \\
\hline & & Solar & \begin{tabular}{|l|c|}
\multicolumn{3}{|c|}{ Labor.atory } \\
Air $110^{\circ} \mathrm{C}$ & $110^{\circ} \mathrm{C}$
\end{tabular} & & & $140 \mathrm{c}$ & 110.0 \\
\hline
\end{tabular}

$\begin{array}{lllllllllllll}451 & 1961.19 & 96 & 26 & 95 & - & b_{-2} & 8_{-4} & 61.25 & 108 & 25\end{array}$

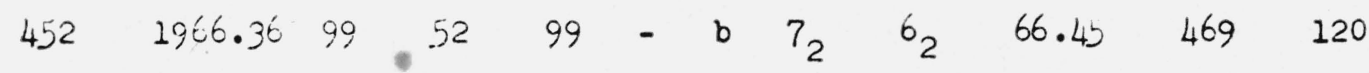
$\begin{array}{llllllllllll}453 & 1967.49 & 99 & 66 & 99 & - & b & 7 & 6 & 67.54 & 1390 & 357\end{array}$ $\begin{array}{lllllllllllll}454 & 1970.0 & 10 & 0 & 52 & - & b & 11 & 11 & 69.35 & 0.8 & 0.6\end{array}$ $\begin{array}{lllll}455 & 1975.43 & 5 & 2 & 32\end{array}-\left\{\begin{array}{llllll}e & 5 & 6 & 72.75 & 0.2 & 0.3 \\ e & 8_{-8}^{0} & 9_{-9}^{-1} & 75.84 & 0.1 & 0.3\end{array}\right.$

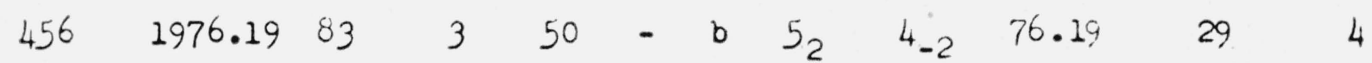
$\begin{array}{lllllllllllll}457 & 1977.6 & 14 & 0 & - & \text { Pcssibly } \mathrm{H}_{2} \mathrm{O} & \mathrm{O} & \mathrm{b} & 6_{5} & 5 \mathrm{~b}\end{array}$

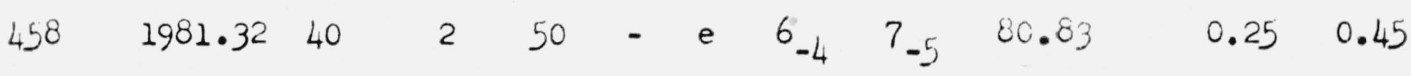
$\begin{array}{llllll}459 & 19,82.1 & 15 & 1 & 27 & -\end{array}$

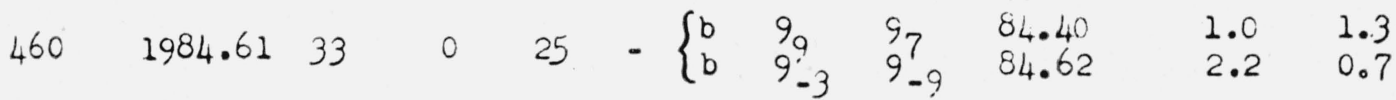

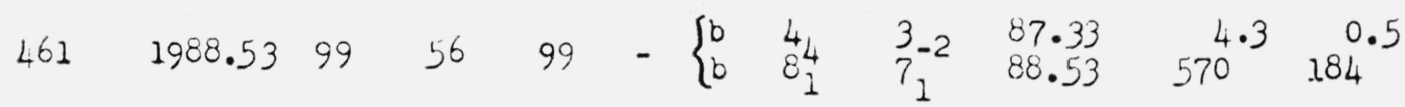

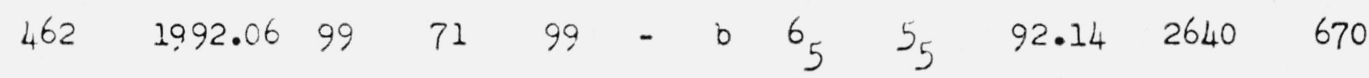

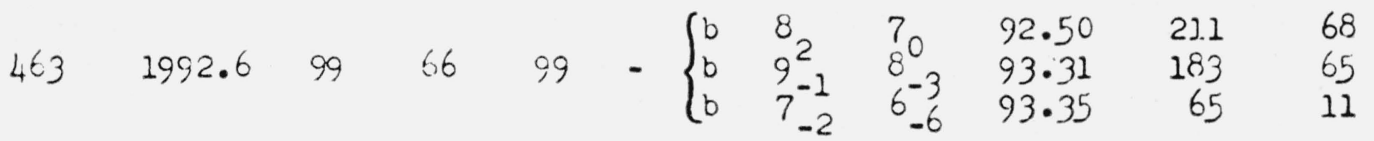

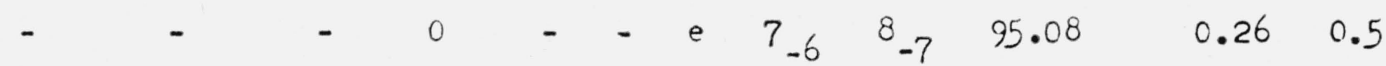
$\begin{array}{llllllllllllll}464 & 1098.94 & 94 & 17 & 85 & - & b & 6 & 5 & 5 & -3 & 98.94 & 144 & 24\end{array}$ $\begin{array}{llllll}4652000.90 & 10 & 0 & 20 & -\end{array}$

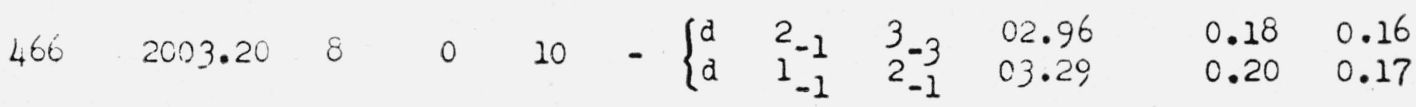
$\begin{array}{llllllllll}- & 0 & - & 0 & \mathrm{e}^{5} & 6_{-3} & 04.14 & 0.31 & 0.4\end{array}$ $\begin{array}{llllllllllll}467 & 2005.8 & 27 & 0 & 12 & - & c & 6_{5} & 5_{5} & 04.8 & 1.3 & 2.7\end{array}$ $\begin{array}{lllllllllllll}468 & 2007.66 & 95 & 26 & 92 & - & b & 9 & 8_{0} & 07.77 & 67.5 & 28\end{array}$

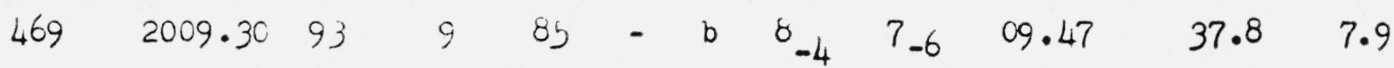

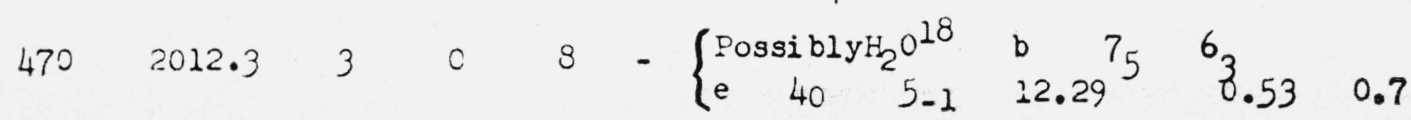


TABLE I-Continued.

\begin{tabular}{|c|c|c|c|c|c|c|c|c|c|c|c|}
\hline \multirow{4}{*}{ 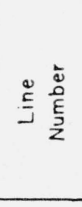 } & \multicolumn{5}{|c|}{ OBSERVED DATA } & & & & \multicolumn{3}{|c|}{ CALCULATED DATA } \\
\hline & \multirow{3}{*}{$\begin{array}{c}\text { Wave } \\
\text { Number } \\
\mathrm{cm}^{-1} \text { (vac) }\end{array}$} & \multicolumn{4}{|c|}{ Intensity } & & \multirow{3}{*}{\multicolumn{2}{|c|}{$\begin{array}{l}\text { Identification } \\
J_{\tau}^{\prime}-J_{\tau}^{\prime \prime}\end{array}$}} & \multirow{3}{*}{$\begin{array}{c}\text { Wave } \\
\text { Number } \\
\mathrm{cm}^{-1}(\mathrm{vac})\end{array}$} & \multicolumn{2}{|c|}{ Iritensity } \\
\hline & & \multirow{2}{*}{ Solar } & \multicolumn{3}{|c|}{ Laboratory } & & & & & & \\
\hline & & & $\begin{array}{l}\text { Air } \\
\text { only } \\
\end{array}$ & 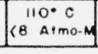 & $\begin{array}{l}110^{\circ} \mathrm{C} \\
8 \text { Aimo-M }\end{array}$ & & & & & $14^{\circ} \mathrm{C}$ & $110 \cdot c$ \\
\hline 471 & 2016.78 & & 73 & - & - & $\mathrm{b}$ & $7_{5}$ & $6_{3}$ & 16.76 & 1235 & 377 \\
\hline 472 & $2018 \cdot 30$ & 99 & 50 & - & - & $\mathrm{b}$ & ${ }^{9} 1$ & ${ }^{8}-1$ & 18.77 & 202 & 83 \\
\hline 473 & 2018.92 & & 34 & - & - & $\mathrm{b}$ & $9-3$ & 8 & 19.18 & 93 & 28 \\
\hline 474 & 2022.95 & 86 & 30 & 9,5 & - & b & $10-1$ & $9-1$ & 23.09 & $52 \cdot 5$ & 34 \\
\hline 475 & 2026.58 & 90 & 10 & 84 & - & $\left\{\begin{array}{l}b \\
a\end{array}\right.$ & $\begin{array}{l}70 \\
53\end{array}$ & $\begin{array}{l}6-4 \\
4-3\end{array}$ & $\begin{array}{l}26.79 \\
27.00\end{array}$ & $\begin{array}{l}41 . \\
23 .\end{array}$ & 8. \\
\hline
\end{tabular}

$\begin{array}{llllll}476 & 2030.03 & 16 & 0^{\circ} & 10 & -\end{array}$

$4772034.0352 \quad 0 \quad 68\left\{\begin{array}{lllllll}b & 11-2 & 10 & -2 & 34.35 & 5.8 & 4.4 \\ b & 8 & 8 & -5 & 34.12 & 0.87 & 0.26 \\ e & 5-4 & 6-5 & 34.13 & 0.63 & 0.82\end{array}\right.$

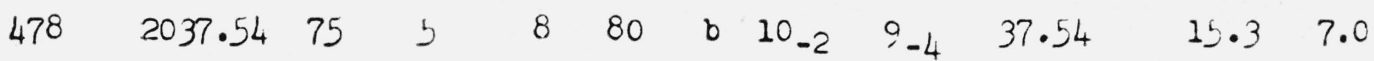

$479 \quad 2041.3593 \quad 31 \quad 70 \quad 95\left\{\begin{array}{cccccc}b & 12 & 11-3 & 41.35 & 4.2 & 4.6 \\ b & 83 & 73 & 41.39 & 384 & 146 \\ b & 84 & 72 & 41.60 & 128 & 48\end{array}\right.$

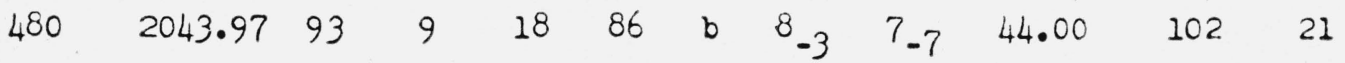

$\begin{array}{lllllllllllll}481 & 2046.58 & 70 & 4 & 13 & 80 & \text { b } & 10 & 9 & 9 & 46.64 & 20.8 & 11\end{array}$

$\begin{array}{lllllllllllll}482 & 2048.7 & ? & 0 & 0 & 7 & \text { e } & 3 & 3_{0} & 4 & 48.66 & 0.71 & 0.76\end{array}$

$\begin{array}{llllllllllllll}483 & 2050.8 & ? & 0 & 0 & 11 & b & 8 & 8 & 8 & 51.08 & 0.37 & 0.12\end{array}$

$\begin{array}{llllllllllllll}484 & 2051.49 & ? & 0 & 0 & 18 & e & 3 & -1 & 4 & -2 & 51.33 & 0.31 & 0.32\end{array}$

$\begin{array}{llllll}485 & 2053.06 \quad ? \quad 0 & 0 & 12\end{array}$

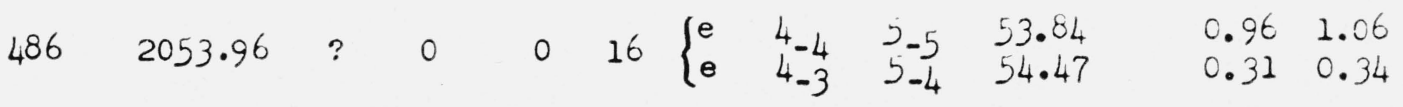

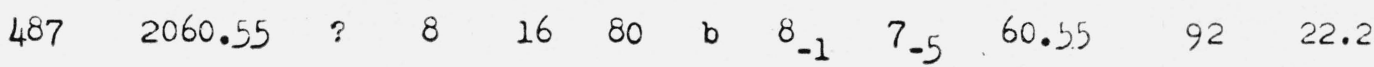

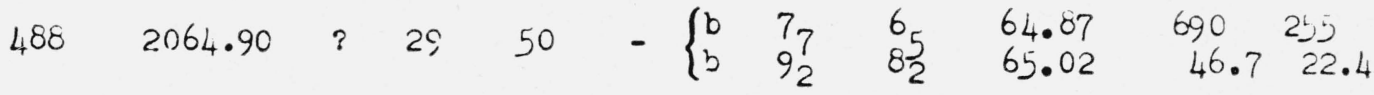

$\begin{array}{llllllllllllll}489 & 2065.83 & ? & 13 & 30 & - & \mathrm{b} & 9_{3} & 8_{1} & 65.91 & 141 & 67.5\end{array}$

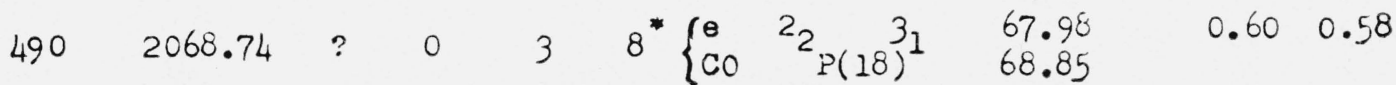


TABLE I-Continued.

\begin{tabular}{|c|c|c|c|c|c|c|c|c|}
\hline \multirow{4}{*}{ 气 } & \multicolumn{4}{|c|}{ OBSERVED DATA } & \multirow{4}{*}{$\begin{array}{l}\text { Identification } \\
J_{\tau}^{\prime}-J_{\tau}^{\prime \prime}\end{array}$} & \multicolumn{3}{|c|}{ CALCULATED DATA } \\
\hline & \multirow{3}{*}{$\begin{array}{c}\text { Wove } \\
\text { Number } \\
\mathrm{cm}^{-1} \text { (voc) }\end{array}$} & \multicolumn{3}{|c|}{ Intensity } & & \multirow{3}{*}{$\begin{array}{l}\text { Wave } \\
\text { Number } \\
\mathrm{cm}^{-1} \text { (vac) }\end{array}$} & \multicolumn{2}{|c|}{ Intensity } \\
\hline & & \multirow{2}{*}{ Solar } & & aboratory & & & & \\
\hline & & & Air & \begin{tabular}{|c|c|}
$110^{\circ} \mathrm{C}$ & $110^{\circ} \mathrm{C}$ \\
$8^{\circ}$ Atmo $\mathrm{M}$ & OA Atmo $\mathrm{M}$ \\
\end{tabular} & & & $14 . \mathrm{C}$ & $110 . \mathrm{c}$ \\
\hline
\end{tabular}

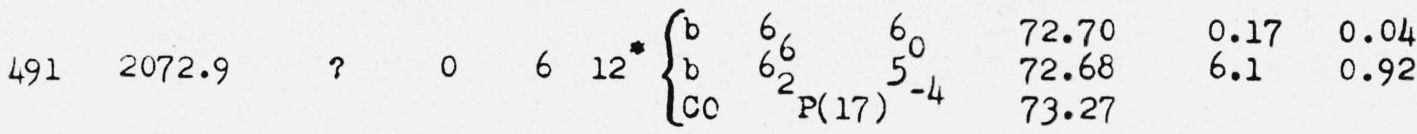

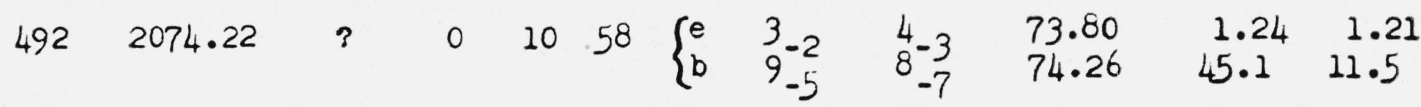

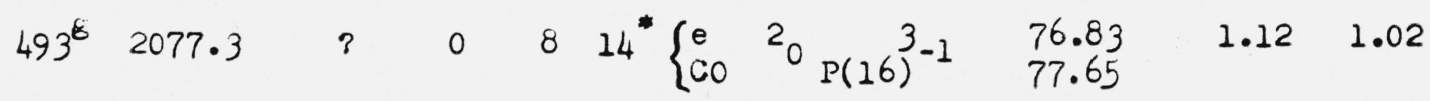

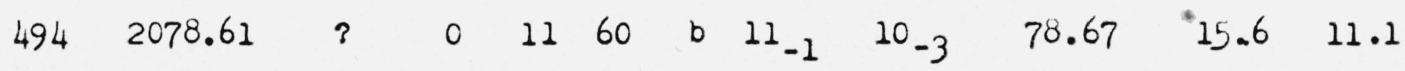
$\begin{array}{lllll}4952081.98 \quad ? \quad 0 & 10 & 3\end{array} 4^{*}\left\{\begin{array}{llllll}c 0 & P(15) & 82.00 \\ b & 10_{-4} & 9 & 82.02 & 8.95 & 3.40\end{array}\right.$ $\begin{array}{llllll}496 & 2086.52 & ? & 0 & 8 & 17\end{array} 7^{*}\left\{\begin{array}{llllll}\mathrm{Co} & \mathrm{P}(14) & 86.32 & \\ \mathrm{~b} & 11-3 & 10_{-5} & 86.52 & 10.6 & 6.4\end{array}\right.$ $\begin{array}{llllllllllll}497 & 2087.54 & ? & 5 & 13 & 61 & \text { b } & 10_{1} & 9_{1} & 87.54 & 45.4 & 28.7\end{array}$

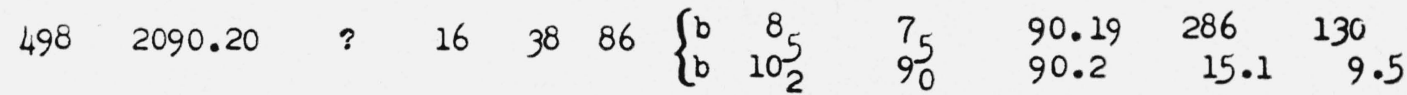

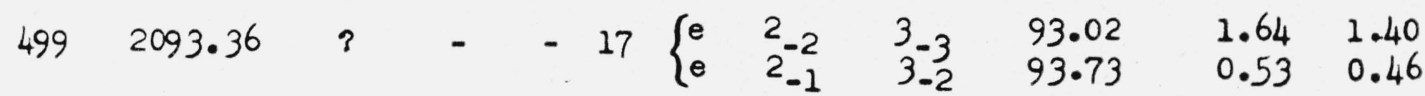
$500 \quad 2095.29 \quad-\quad-23^{*}\left\{\begin{array}{llllll}\mathrm{b} & 8 & 8 & 85.14 & 0.90 & 0.31 \\ 0 & 44^{3} & 43^{-3} & 95.28 & 0.72 & 1.01\end{array}\right.$

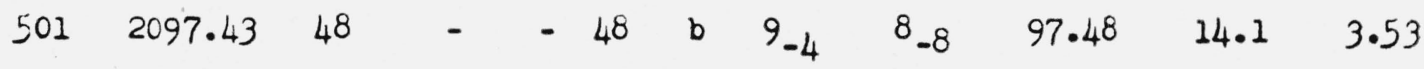
$\begin{array}{lllllllllllll}502 & 2100.46 & 50 & - & - & 61 & \mathrm{~b} & 9_{-2} & 8_{-6} & 00.46 & 13.9 & 4.20\end{array}$

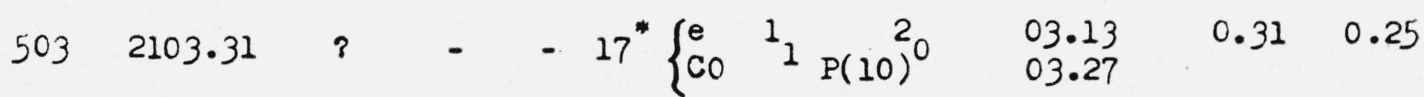
$\begin{array}{llllllllllll}- & 2105.93^{\dagger} & 20 & - & - & & - & 5_{5} & 4_{-1} & 05.86 & 5.35 & 0.77\end{array}$

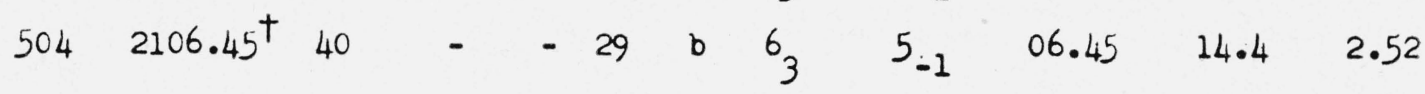

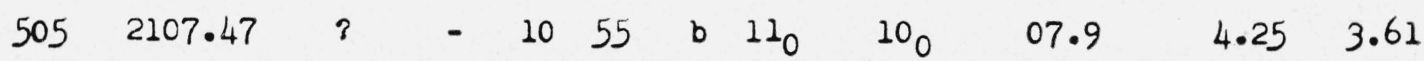

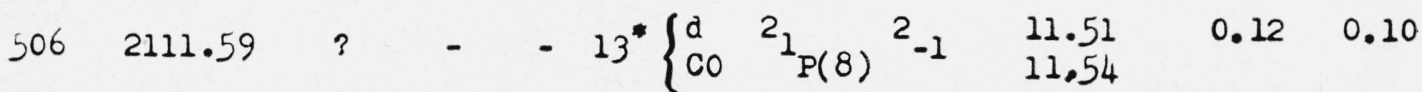
$5072114.86 \quad 80 ?-4499\left\{\begin{array}{cccccc}d & 3-3 & 2-1 & 14.28 & 0.24 & 0.20 \\ e & 10 & 2-1 & 14.45 & 1.01 & 0.82 \\ b & 11 & 10^{-1} & 14.50 & 12.6 & 10.7 \\ b & 95 & 83 & 15.18 & 107.5 & 62.5 \\ d & 2-1 & 1-1 & 16.47 & 0.29 & 0.26\end{array}\right.$ 
TABLE I-Continued.

\begin{tabular}{|c|c|c|c|c|c|c|c|c|}
\hline \multirow{4}{*}{ 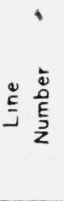 } & \multicolumn{4}{|c|}{ OBSERVED DATA } & \multirow{4}{*}{$\begin{array}{l}\text { Identification } \\
J_{\tau}^{\prime}-J_{\tau}^{\prime \prime}\end{array}$} & \multicolumn{3}{|c|}{ CALCULATED DATA } \\
\hline & \multirow{3}{*}{$\begin{array}{c}\text { Wave } \\
\text { Number } \\
\mathrm{cm}^{-1} \text { (vac) }\end{array}$} & \multicolumn{3}{|c|}{ Intensity } & & \multirow{3}{*}{$\begin{array}{c}\text { Wove } \\
\text { Number } \\
\mathrm{cm}^{-1} \text { (vac) }\end{array}$} & \multicolumn{2}{|c|}{ Intensity } \\
\hline & & \multirow{2}{*}{ Solar } & & aboratory & & & & \\
\hline & & & $\begin{array}{l}\text { Air } \\
\text { only }\end{array}$ & 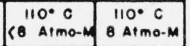 & & & $14^{\circ} \mathrm{C}$ & $110^{\circ} \mathrm{c}$ \\
\hline
\end{tabular}

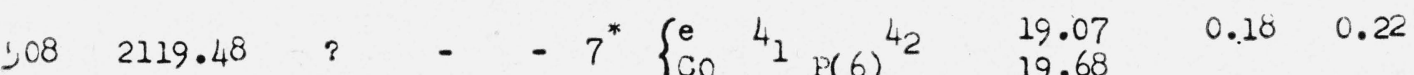

$5092121.5420 \quad-\quad 28\left\{\begin{array}{llllll}b & 72 & 6 & 21.48 & 6.5 & 1.37 \\ e & 42 & 4_{1}^{-2} & 21.73 & 0.53 & 0.48\end{array}\right.$

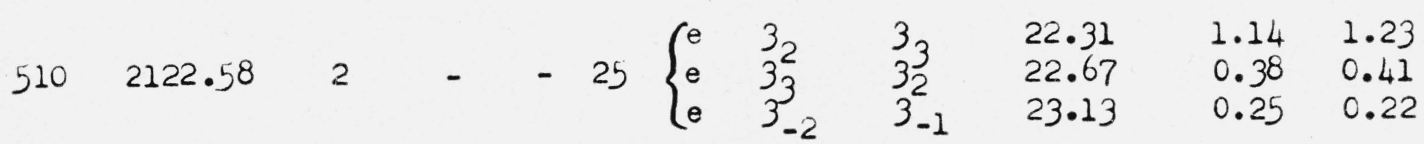

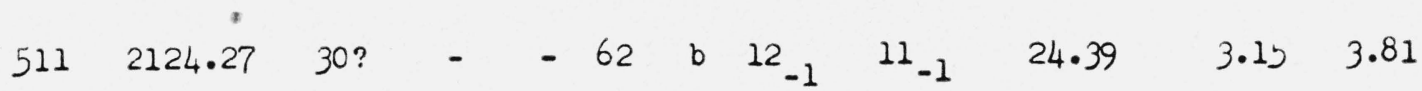

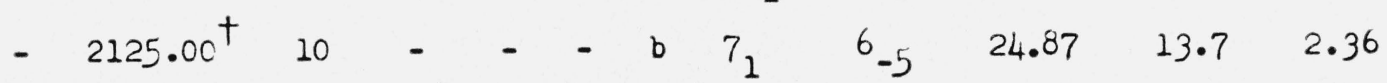

$5122127.57 \quad ? \quad-\quad-5^{*}$ CO $P(4) \quad 27.68$

$513 \quad 2129.54 \quad 39 \quad-\quad-5$

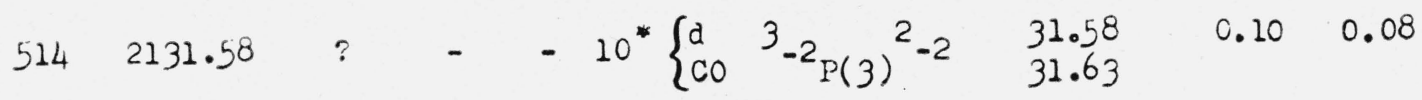

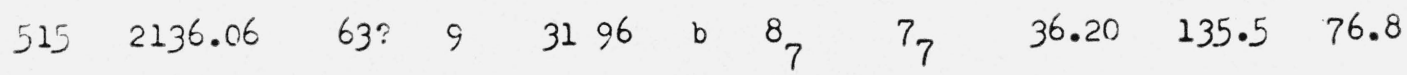

$\begin{array}{llll}56 & 2137.12 & 55 & 0\end{array}-75\left\{\begin{array}{llllll}\mathrm{b} & 6 & 5 & 36.86 & 3.5 & 0.60 \\ \mathrm{~b} & 84 & 7-2 & 37.35 & 18.3 & 4.83\end{array}\right.$

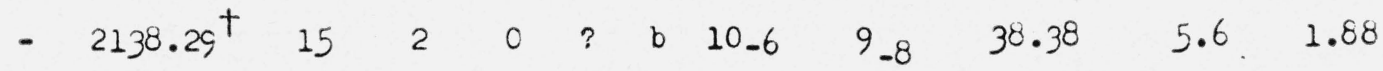

$\begin{array}{llllllllllll}517 & 2139.30 & ? & 4 & 30 & 95 & \mathrm{~b} & 10_{3} & 93 & 39.37 & 25.7 & 19.8\end{array}$

$5182141.3 \quad ? \quad 0 \quad-2$

$\begin{array}{lllllllllllll}519 & 2142.4 & 8 & 0 & - & 2 & \mathrm{e} & 2_{1} & 2_{2} & 41.94 & 0.52 & 0.47\end{array}$

$520 \quad 2145.41 \quad 52 \quad 0 \quad 1277\left\{\begin{array}{cccccc}e & 2 & 2 & 44.63 & 1.57 & 1.40 \\ b & 10^{2} & 9_{-3} & 45.64 & 20.3 & 7.8\end{array}\right.$

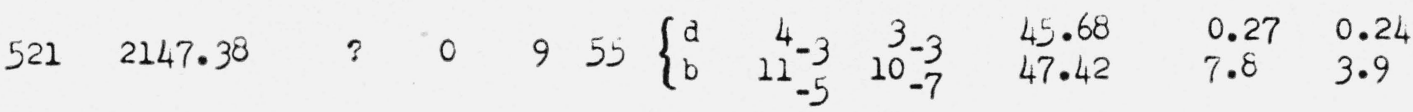

$522 \quad 2151.24 \quad 0 \quad-20\left\{\begin{array}{llllll}d & 2 & 1 & 48.10 & 0.08 & 0.06 \\ e & 1_{0} & 1_{1} & 51.04 & 0.39 & 0.31\end{array}\right.$

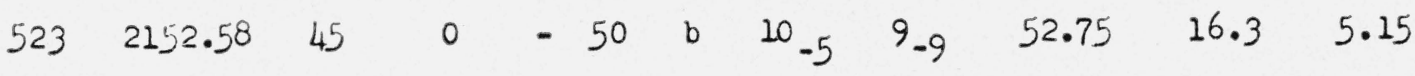

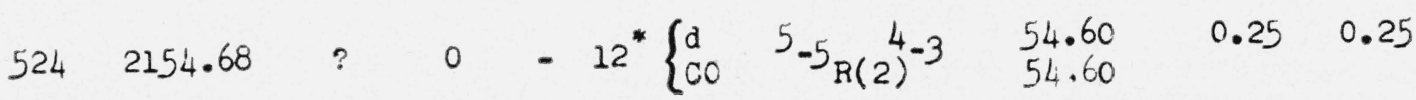


TABLE I-Continued.

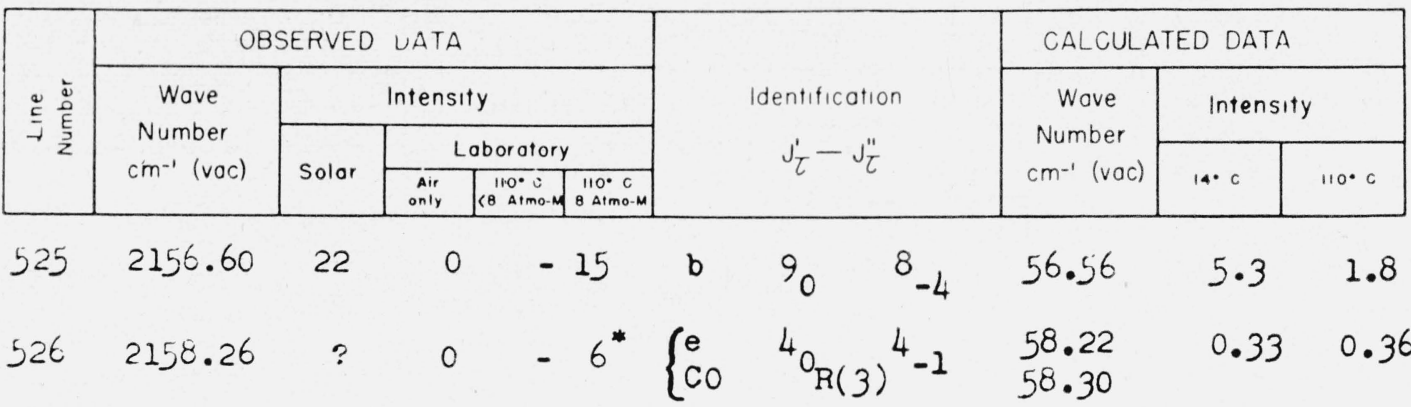

$\begin{array}{lllllllllll}527 & 2161.75 & ? & 0 & -84 & b & 9 & 8 & 61.88 & 51 & 36\end{array}$

$528\left\{\begin{array}{ll}2162.90^{+} \\ 2163.46^{+} & 10 \\ 15\end{array}\right\} \quad-69\left\{\begin{array}{llllll}b & 11 & 10_{2} & 62.90 & 2.48 & 2.58 \\ b & 111_{3} & 10_{1} & 63.46 & 7.44 & 7.73\end{array}\right.$

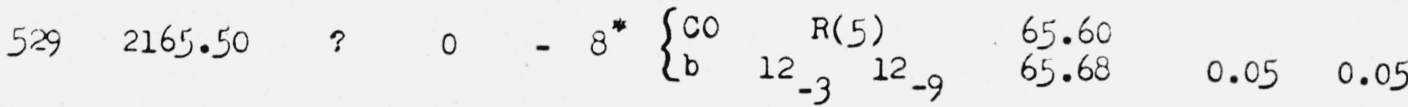

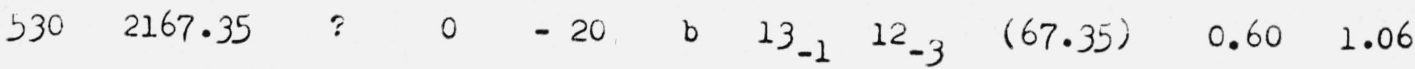

$\begin{array}{llllllll}531 & 2169.17 & ? & 0 & -6^{*} & & & \end{array}$

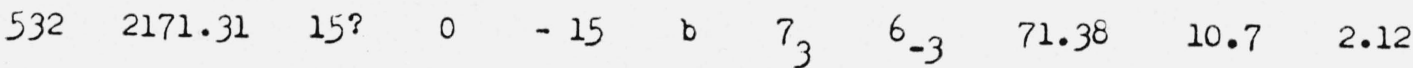

$5332172.43 \quad ? \quad 0 \quad-16^{*}\left\{\begin{array}{llllll}e & 2 & 2 & 72.06 & 0.60 & 0.49 \\ d & 60 & 5-1 & 72.35 & 0.07 & 0.08 \\ c 0 & & -k(7) & -4 & 72.76 & \end{array}\right.$

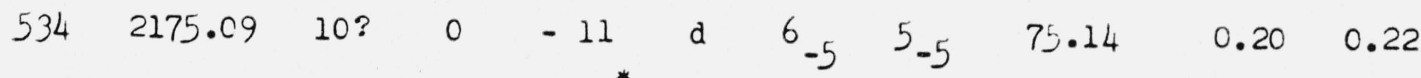

$\begin{array}{lllllllll}535 & 2176.45 & 8 & 0 & -7^{*} & \mathrm{CO} & \mathrm{R}(8) & 76.28\end{array}$

$\begin{array}{llllllllllll}536 & 2178.99 & ? & 0 & -8 & \mathrm{~d} & 4_{-1} & 3_{-1} & 78.91 & 0.17 & 0.16\end{array}$

$\begin{array}{lllllllll}537 & 2179.87 & ? & 0 & -10^{*} & \text { CO } & K(9) & 79.77\end{array}$

$\begin{array}{llllllllllll}538 & 2181.41 & ? & 0 & -40 & b & 10 & 9 & 9 & 81.61 & 8.84 & 3.98\end{array}$

$5392183.33 \div 2 \quad 6^{2} \quad 6^{*}$ co $R(10) \quad 83.22$

$\begin{array}{lll}540 & 2185.41\end{array} \quad-38\left\{\begin{array}{cccccc}b & 8 & 7 & 85.24 & 2.0 & 0.42 \\ b & 12 & 11-6 & 85.37 & 1.9 & 2.75\end{array}\right.$

$5412187.05 ? \quad-28 *\left\{\begin{array}{llllll}b & 12 & 11 & 87.0 & 0.65 & 0.92 \\ b & 102 & 9 & 87.04 & 16.6 & 15.8\end{array}\right.$

$\begin{array}{llllllllllll}542 & 2189.69 & 3 & 0 & -11 & d & 7 & 6_{-5} & 89.52 & 0.13 & 0.16\end{array}$

$\begin{array}{lllll}543 & 2190.4 & * & -8^{*}\end{array} \quad\left\{\begin{array}{llllll}c 0 & R(12) & 90.02 & \\ d & 7 & 6 & 90.7 & 0.04 & 0.05\end{array}\right.$

$\begin{array}{llllllllllll}544 & 2191.83 & 3 & 0 & - & 2 & d & 3_{2} & 2_{2} & 91.34 & 0.086 & 0.07\end{array}$ 
TABIE I-Continued.

\begin{tabular}{|c|c|c|c|c|c|c|c|c|}
\hline \multirow{4}{*}{ 站 } & \multicolumn{4}{|c|}{ OBSERVED DATA } & \multirow{4}{*}{$\begin{array}{l}\text { Identification } \\
J_{\tau}^{\prime}-J_{\tau}^{\prime \prime}\end{array}$} & \multicolumn{3}{|c|}{ CALCULATED DATA } \\
\hline & \multirow{3}{*}{$\begin{array}{c}\text { Wave } \\
\text { Number } \\
\mathrm{cm}^{-1} \text { (voc) }\end{array}$} & \multicolumn{3}{|c|}{ Intensity } & & \multirow{3}{*}{$\begin{array}{c}\text { Wove } \\
\text { Number } \\
\mathrm{cm}^{-1} \text { (voc) }\end{array}$} & \multicolumn{2}{|c|}{ Intensity } \\
\hline & & \multirow[b]{2}{*}{ Solor } & \multicolumn{2}{|c|}{ Laboratory } & & & & \\
\hline & & & Alr & 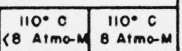 & & & $14^{\circ} \mathrm{C}$ & $110 \cdot \mathrm{c}$ \\
\hline
\end{tabular}

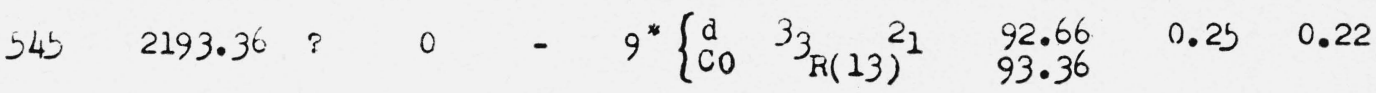

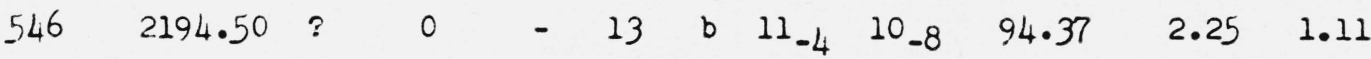

$5472196.69 ? \quad 0 \quad-\quad 5^{*}$ Co $R(14) \quad 96.66$

$\begin{array}{lllllllllllll}548 & 2198.63 & ? & 0 & - & 11 & \text { e } & 2 & -1 & 10 & 98.42 & 0.40 & 0.31\end{array}$

$\begin{array}{llllllllllll}549 & 2200.29 & 0 & -25^{*} & \mathrm{~b} & 11 & 10_{-9} & 00.4 & 5.5 & 2.23\end{array}$

$550 \quad 2201.2 \quad ? \quad 0 \quad-4$

$5512202.0 \quad ? \quad 0 \quad-\quad 2$

$5522203.23 \div 0 \quad-4^{*}$ Co $R(16) \quad 03.16$

$\begin{array}{llllllllllllll}553 & 2205.19 & ? & 0 & - & 60 & \text { b } & 99 & 8 & 9 & 05.30 & 19.3 & 18.1\end{array}$

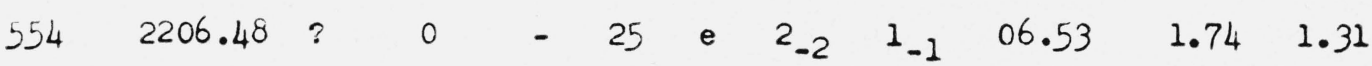

$5552208.83 ? \quad 0 \quad-16\left\{\begin{array}{lllllll}e & 2 & 1 & 1 & 08.65 & 1.12 & 0.86 \\ b & 11_{-6} & 10_{-10}^{1} & 08.96 & 1.88 & 0.75\end{array}\right.$

$\begin{array}{llllllllllllll}556 & 2209.9 & ? & 0 & - & 5 & b & 13 & 12 & -1 & (09.9) & 0.42 & 0.87\end{array}$

$\begin{array}{lllllllllllll}557 & 2210.4 & 3 & 0 & - & 5 & \mathrm{~b} & 8 & 8_{2} & 7_{-4} & 10.76 & 2.38 & 0.58\end{array}$

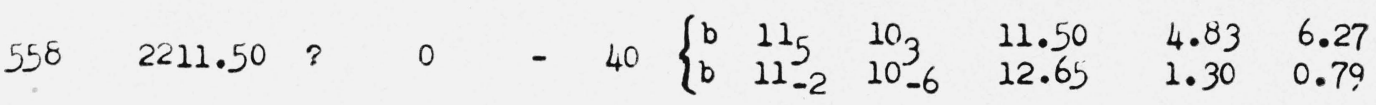

$\begin{array}{lllllllllllll}559 & 2214.0 & ? & 0 & - & 8 & \text { e } & 30 & 2_{1} & 14.09 & 0.80 & 0.70\end{array}$

$\begin{array}{llllll}560 & 2214 \cdot 3 & ? & 0 & - & 8\end{array}$

$5612215.80 ? 0 \quad-5$

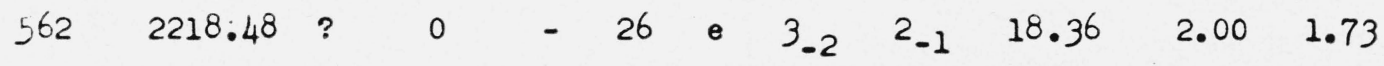

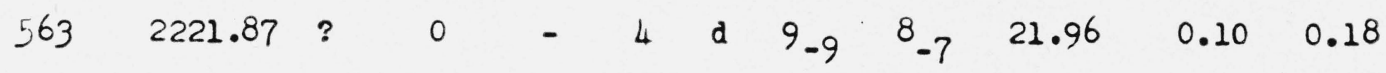

$5642223.61 \quad ? \quad 0 \quad-4$ 
TABLE $1-$ Continued.

\begin{tabular}{|c|c|c|c|c|c|c|c|c|c|c|c|}
\hline \multirow{3}{*}{ 竞离 } & \multicolumn{5}{|c|}{ OBSERVED DATA } & & & & \multicolumn{3}{|c|}{ CALCULATED DATA } \\
\hline & \multirow{3}{*}{$\begin{array}{c}\text { Wave } \\
\text { Number } \\
\mathrm{cm}^{-1} \text { (vac) }\end{array}$} & \multicolumn{4}{|c|}{ Intensity } & \multirow{3}{*}{\multicolumn{3}{|c|}{$\begin{array}{l}\text { Identification } \\
J_{\tau}^{\prime}-J_{\tau}^{\prime \prime}\end{array}$}} & \multirow{3}{*}{$\begin{array}{c}\text { Wave } \\
\text { Number } \\
\mathrm{cm}^{-1} \text { (vac) }\end{array}$} & \multicolumn{2}{|c|}{ Intensity } \\
\hline & & \multirow{2}{*}{ Solar } & \multicolumn{3}{|c|}{ Laboratory } & & & & & $14^{\circ} \mathrm{c}$ & $1100^{\circ}$ \\
\hline & & & only & & & & & & & & \\
\hline 565 & 2225.84 & ? & 0 & - & & $\begin{array}{l}b \\
e\end{array}$ & $\begin{array}{l}6 \\
3_{-3}^{5}\end{array}$ & ${ }^{5}-2$ & $\begin{array}{l}25.45 \\
25.87\end{array}$ & $\begin{array}{l}1.55 \\
0.72\end{array}$ & $\begin{array}{l}0.29 \\
0.57\end{array}$ \\
\hline 566 & 2227.30 & $?$ & 0 & - & 16 & $\begin{array}{l}d \\
d \\
b\end{array}$ & $\begin{array}{l}4_{4} \\
4_{3} \\
6_{3}\end{array}$ & $\begin{array}{l}3 \\
3 \\
5 \\
5 \\
-5\end{array}$ & $\begin{array}{l}27.09 \\
27.45 \\
27.59\end{array}$ & $\begin{array}{l}0.07 \\
0.22 \\
0.06\end{array}$ & $\begin{array}{l}0.08 \\
0.24 \\
0.07\end{array}$ \\
\hline 567 & 2231.01 & $?$ & 0 & - & 40 & $b$ & $10_{7}$ & $9_{7}$ & 31.01 & 7.10 & 9.0 \\
\hline 568 & 2232.98 & $?$ & 0 & - & 10 & e & ${ }^{3}-1$ & $2_{0}$ & 33.13 & 0.55 & 0.45 \\
\hline 569 & 2235.62 & $?$ & 0 & - & 25 & $\mathrm{~b}$ & $12{ }_{3}$ & $11_{3}$ & 35.62 & 1.30 & 2.30 \\
\hline 570 & 2237.76 & $?$ & 0 & - & 10 & e & ${ }^{4}-3$ & $3-2$ & 37.64 & 0.66 & 0.58 \\
\hline 571 & 2230.30 & $?$ & 0 & - & 8 & & & & & & \\
\hline
\end{tabular}

†, Frequency obtalned from solar spectme; $g$, probably due to $\infty_{2}$;

$h$, this line looks like an unresolved doublet; $j$, obviously consists of several unresolved components; *, intensity variable, due to $\infty$ contaminant.

TABLE 2. Energy Levels of $\mathrm{H}_{2} \mathrm{O}\left(\mathrm{cm}^{-1}\right)$.

\begin{tabular}{|c|c|c|c|c|c|c|c|}
\hline \multirow{2}{*}{$\mathrm{J}_{\tau}$} & \multicolumn{3}{|c|}{$\mathrm{V}_{1} \mathrm{~V}_{2} \mathrm{~V}_{3}$} & \multirow{2}{*}{${ }^{J_{\tau}}$} & \multicolumn{3}{|c|}{$\mathrm{V}_{1} \mathrm{~V}_{2} \mathrm{~V}_{3}$} \\
\hline & 000 & 010 & 020 & & $\infty 00$ & 010 & 020 \\
\hline 0 & 0.00 & 1594.59 & 3151.53 & 4 & 222.04 & 1817.35 & 3375.3 \\
\hline $1-1$ & 23.79 & 1618.41 & 3175.4 & 4 & 224.83 & 1821.63 & 3381.9 \\
\hline $1_{0}$ & 37.13 & 1634.94 & 3196.2 & 4 & 275.48 & 1875.42 & 3438.6 \\
\hline 1 & 42.37 & 1640.48 & 3202.0 & & 300.38 & 1907.94 & 3482.0 \\
\hline & & & & & 315.73 & 1923.04 & 3495.85 \\
\hline $2-2$ & 70.06 & 1664.93 & 3222.06 & 4 & 382.49 & 2004.89 & 3597.80 \\
\hline $2-1$ & 79.48 & 1677.07 & 3237.77 & $4 \frac{1}{2}$ & 383.86 & 2006.12 & 3598.8 \\
\hline $2_{0}$ & 95.17 & 1693.62 & 3255.26 & $4_{3}$ & 488.16 & 2129.60 & 3746.8 \\
\hline 21 & 134.91 & 1742.51 & 3316.0 & $4_{4}$ & 488.17 & 2129.60 & 3746.8 \\
\hline $2_{2}$ & 136.15 & 1743.64 & 3317.0 & & & & \\
\hline & & & & $5-5$ & 325.36 & 1920.70 & 3479.1 \\
\hline $3-3$ & 136.77 & 1731.92 & 3289.27 & & 326.59 & 1922.80 & 34.82 .5 \\
\hline${ }^{3}-2$ & 142.27 & 1739.63 & 3300.1 & $5-3$ & 399.45 & 2000.80 & 3565.5 \\
\hline${ }^{3}-1$ & 173.36 & 1772.30 & 3334.55 & $5-2$ & 416.12 & 2024.24 & 3598.6 \\
\hline 30 & 206.28 & 1813.87 & 3387.8 & $5-1$ & 446.50 & 2054.07 & 3627.0 \\
\hline 3 & 212.16 & 1819.16 & 3392.90 & 50 & 504.00 & 2126.44 & 3719.2 \\
\hline $3_{2}$ & 285.25 & 1907.60 & 3500.4 & 51 & 508.79 & 2130.52 & 3722.55 \\
\hline $3_{3}^{2}$ & 285.40 & 1907.71 & 3500.5 & $5_{2}$ & 610.12 & 2251.67 & 3868.3 \\
\hline & & & & & 610.34 & 2251.83 & 3868.5 \\
\hline & & & & 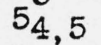 & 742.10 & 2406.24 & 4052.8 \\
\hline
\end{tabular}


TABLE 2 - Continued.

\begin{tabular}{|c|c|c|c|c|c|c|c|}
\hline \multirow{2}{*}{${ }^{\mathrm{J}}$} & \multicolumn{3}{|c|}{$V_{1} V_{2} V_{3}$} & \multirow{2}{*}{${ }^{\mathrm{J}}{ }_{\tau}$} & \multicolumn{3}{|c|}{$\mathrm{V}_{1} \mathrm{~V}_{2} \mathrm{~V}_{3}$} \\
\hline & 000 & 010 & 020 & & 000 & 010 & 020 \\
\hline $6-6$ & 446.71 & 2041.73 & 3600.4 & $9-3$ & 1283.02 & 2904.82 & \\
\hline $6-5$ & 447.24 & 2042.73 & 3602.0 & $9_{-2}^{-3}$ & 1341.03 & 2983.43 & \\
\hline $6-4$ & 542.87 & 2146.39 & 3713.1 & $9^{-2}$ & 1360.56 & 2999.45 & \\
\hline $6-3$ & 552.92 & 2161.31 & 3736.2 & $9_{0}^{-1}$ & 1475.14 & 3139.65 & \\
\hline $6-2$ & 602.67 & 2211.23 & 3784.3 & $9_{1}$ & 1477.46 & 3141.55 & \\
\hline $6-1$ & 648.97 & 2271.60 & 3864.8 & $9 \frac{1}{2}$ & 1631.44 & 3321.0 & \\
\hline & 661.54 & 2282.56 & 3873.55 & 93 & 1631.58 & 3321.10 & \\
\hline 61 & 756.76 & 2398.39 & 4015.1 & 945 & 1810.76 & 3526.77 & \\
\hline 62 & 757.70 & 2399.27 & 4015.8 & 9,5 & 2009.99 & 3752.58 & \\
\hline $6_{3}$ & 888.67 & 2552.95 & 4197.4 & $98, ?$ & 2225.56 & 3994.39 & \\
\hline $6_{4}$ & 888.71 & 2552.98 & 4197.4 & 78,9 & 2020.00 & 3994.39 & \\
\hline 65,6 & 1045.15 & 2734.24 & 24411.0 & $10-10$ & 1114.59 & 2705.20 & 4260.7 \\
\hline $7-7$ & 586.28 & 2180.68 & 3738.6 & $10_{-9}^{-10}$ & 1114.59 & 2705.23 & \\
\hline $7-6$ & 586.43 & 2181.27 & 3739.5 & $10-8$ & 1293.22 & 2903.38 & \\
\hline $7^{-0}-5$ & 704.20 & 2309.89 & 3879.05 & $10^{-0}$ & 1293.80 & 2904.68 & \\
\hline $7-4$ & 709.50 & 2318.48 & 3894.5 & $10_{-6}^{-7}$ & 1438.19 & 3058.60 & \\
\hline $7_{-3}^{-4}$ & 782.41 & 2392.38 & 3967.4 & $10_{-5}^{-6}$ & 1446.23 & 3072.95 & \\
\hline $7-2$ & 816.65 & 2440.06 & 4038.7 & $10_{-4}^{-5}$ & 1538.31 & 3162.53 & \\
\hline $7^{-2}$ & 842.36 & 2462.87 & 4053.0 & $10-3$ & 1581.53 & 3224.80 & \\
\hline $7_{0}^{-1}$ & 927.76 & 2569.66 & 34187.8 & $10_{-2}$ & 1616.49 & 3253.91 & \\
\hline $7_{1}$ & 931.23 & 2572.11 & 4190.8 & $10_{-1}^{-2}$ & 1719.36 & 3383.65 & \\
\hline 72 & 1059.68 & 2724.15 & & $10_{0}^{-1}$ & 1724.80 & 3387.67 & \\
\hline $7_{3}^{2}$ & 1059.89 & 2724.30 & & 10 & 1875.24 & 3565.00 & \\
\hline 74,5 & 1216.39 & 2905.43 & & $10_{2}^{1}$ & 1875.72 & 3565.3 & \\
\hline 76,7 & 1394.85 & 3110.02 & & $10_{3,4}^{2}$ & 2054.55 & 3770.95 & \\
\hline & & & & $10_{5,6}^{0,4}$ & 2254.55 & 3997.80 & \\
\hline $8-8$ & 744.09 & 2337.61 & 3894.4 & 107,8 & 2471.59 & 4241.0 & \\
\hline $8-7$ & 744.14 & 2337.84 & 3894.8 & 10,10 & 2702.09 & & \\
\hline $8-6$ & 882.97 & 2490.42 & 4063.9 & & & & \\
\hline $8-5$ & 885.64 & 2495.25 & 4071.6 & $11-11,-10$ & 1327.25 & 2916.09 & 4470.1 \\
\hline $8-4$ & 983.09 & 2595.9 & & $11-9$ & 1525.02 & 3135.9 & \\
\hline $8-3$ & 1006.14 & 2630.28 & 4224.8 & $11_{-8}$ & 1525.31 & 3136.66 & \\
\hline $8-2$ & 1050.20 & 2670.75 & & $11_{-7}$ & 1690.85 & 3315.0 & \\
\hline $8_{-1}^{-2}$ & 1122.78 & 2764.75 & 4383.9 & $11_{-6}$ & 1695.24 & 3323.55 & \\
\hline 80 & 1131.88 & 2771.67 & & $11_{-5}^{-0}$ & 1813.47 & 3441.22 & \\
\hline 81 & 1255.19 & 2919.76 & & $11_{-4}^{-4}$ & 1843.32 & 3487.59 & \\
\hline & 1255.98 & 2920.26 & & $11-3$ & 1899.21 & 3532.75 & \\
\hline $8_{3,4}^{2}$ & 1411.59 & 3101.28 & & $11_{-2}$ & 1986.08 & 3650.84 & \\
\hline 8,6 & 1590.70 & 3306.58 & & $11_{-1}^{-2}$ & 1999.34 & 33660.20 & \\
\hline 87,8 & 1789.09 & 3531.05 & & 110 & 2143.01 & 3832.7 & \\
\hline & & & & $11_{1}$ & 2144.46 & 3833.86 & \\
\hline $9-9$ & 920.20 & 2512.37 & 4068.8 & 112 & 2322.20 & 4038.62 & \\
\hline $9_{-8}^{-9}$ & 920.22 & 2512.50 & & $11_{3}$ & 2322.25 & 4038.70 & \\
\hline $9^{-8}$ & 1079.16 & 2688.26 & 4266.7 & $11_{4}$ & 2522.46 & 4266.05 & \\
\hline & 1080.51 & 2690.73 & & 116.7 & 2740.73 & & \\
\hline & 1202.04 & 2818.40 & & $11_{8} 9$ & 2973.07 & & \\
\hline & 1216.37 & 2841.57 & & ${ }^{11_{10,11}}$ & 3216.6 & & \\
\hline
\end{tabular}


TABLE 2 - Continued.

\begin{tabular}{|c|c|c|c|c|c|c|c|}
\hline \multirow{2}{*}{$\mathrm{J}_{\tau}$} & \multicolumn{3}{|c|}{$\mathrm{V}_{1} \mathrm{~V}_{2} \mathrm{~V}_{3}$} & \multirow{2}{*}{$\mathrm{J}_{\tau}$} & \multicolumn{3}{|c|}{$V_{1} V_{2} V_{3}$} \\
\hline & 000 & 010 & 020 & & 000 & 010 & 020 \\
\hline $12-12-11$ & 1558.07 & 3144.77 & & $14-2$ & 3101.65 & & \\
\hline $12_{-10}^{-12,-11}$ & 1774.75 & 3386.27 & & $14_{-1}^{-2}$ & 3264.2 & & \\
\hline $12-9$ & 1774.88 & 3386.53 & & 140 & 3266.36 & & \\
\hline $12-8$ & 1960.38 & 3587.87 & & 141 & 3465.18 & & \\
\hline $12-7$ & 1962.60 & 3592.71 & & 142 & 3465.4 & & \\
\hline $12-6$ & 2106.7 & & & ${ }^{14} 3,4$ & & & \\
\hline $12_{-5}$ & 2124.84 & 3771.13 & & & & & \\
\hline $12_{-4}^{-4}$ & 2205.95 & & & $15-15,-14$ & 2358.58 & 3937.87 & \\
\hline $12-3$ & 2275.65 & 3940.56 & & $\begin{array}{l}-15,-14 \\
15-13,-12\end{array}$ & 2631.6 & & \\
\hline $12-2$ & 2300.94 & & & $15_{-11}^{-13,-12}$ & 2872.56 & & \\
\hline $12-1$ & 2434.14 & 4123.73 & & $15_{-10}^{-11}$ & 2872.9 & & \\
\hline $12_{0}$ & 2437.84 & & & $15-9$ & 3081.2 & & \\
\hline 12 & 2613.26 & 4329.83 & & $15_{-8}^{-8}$ & 3084.2 & & \\
\hline $12_{2}^{1}$ & 2613.49 & 4330.0 & & $15-7$ & 3252.0 & & \\
\hline $12_{3,4}^{2}$ & 2813.94 & 4557.87 & & $15_{-6}^{-1}$ & 3277.0 & & \\
\hline 12,6 & 3033.17 & & & $15_{-5}^{-0}$ & 3365.0 & & \\
\hline 1278 & 3267.2 & & & $15_{-4}^{-0}$ & 3446.0 & & \\
\hline $12_{9}^{\prime}, 10$ & 3512.8 & & & $15-3$ & 3473.0 & & \\
\hline $12_{11,12}^{9}$ & 3767.1 & & & $15-2$ & 3624.0 & & \\
\hline 1373 & 1806.94 & 3391.46 & & $15^{-2}-1$ & 3628.7 & & \\
\hline $\begin{array}{l}10-13,-12 \\
13-11\end{array}$ & 2042.5 & 3654.28 & & $15_{0}^{\circ}$ & 3824.8 & & \\
\hline $13-11$ & 2042.5 & & & $15_{1}$ & 3826.1 & & \\
\hline $13-9$ & 2247.0 & 3877.9 & & $15_{2,3}^{2}$ & 4045.8 & & \\
\hline $13_{-8}^{-8}$ & 2248.24 & & & & 2661.2 & 4237.5 & \\
\hline $13-7$ & 2415.95 & & & & 2953.0 & 4200 & \\
\hline $13-6$ & 2426.2 & & & $16-14,-13$ & 3211.5 & & \\
\hline $13-5$ & 2534.14 & & & & 3437.7 & & \\
\hline $13-4$ & 2586.5 & & & & 3439.7 & & \\
\hline $13-3$ & 2629.54 & & & $16^{-9}$ & 3640.3 & & \\
\hline $13-2$ & 2748.4 & & & $16-7$ & 3657.1 & & \\
\hline 13 & 2756.61 & 4443.0 & & & & & \\
\hline $130^{-1}$ & 2927.38 & & & $17-17-16$ & 2931.5 & 4554.6 & \\
\hline $13_{1}$ & 2928.45 & 4644.0 & & $\begin{array}{l}17-17,-16 \\
-15,-14\end{array}$ & 3291.0 & & \\
\hline $13_{2,3}^{+}$ & 3128.25 & & & $17-13,-12$ & 3567.5 & & \\
\hline $13_{4,5}$ & 3348.2 & & & $17-11$ & 3011.7 & & \\
\hline $13_{6,7}$ & 3584.0 & & & $17^{-11}-10$ & $\begin{array}{l}3812.8 \\
4047.1\end{array}$ & & \\
\hline $14-14,-13$ & 2073.81 & 3655.74 & & $17_{-8}^{-9}$ & 4057.3 & & \\
\hline $14-12,-11$ & 2328.2 & 3940.1 & & & & & \\
\hline $14-10$ & 2551.0 & & & $18-18,-17$ & 3319.4 & & \\
\hline $14-9$ & 2551.5 & & & $18-16,-15$ & 3648.0 & & \\
\hline $14-8$ & 2740.5 & & & $18-14,-13$ & 3940.8 & & \\
\hline $14-7$ & 2745.5 & & & $18-12,-11$ & 4201.8 & & \\
\hline $14-6$ & 2883.5 & & & & & & \\
\hline $14-5$ & 2919.5 & & & & & & \\
\hline $14-4$ & 2983.6 & & & & - & & \\
\hline $14-3$ & 3085.0 & & & & & & \\
\hline
\end{tabular}


Table 3. Series regularities in the $P_{1, \overline{1}}, P_{\overline{1}, \overline{1}}, P_{\overline{1}, 1}$, and $P_{\overline{3} 1}$ branches of $\nu_{2}$

\begin{tabular}{|c|c|c|c|c|c|c|c|c|c|c|c|c|c|c|c|c|c|c|}
\hline Branch & $(J+\tau)^{\prime}$ & $J^{\prime}=0$ & 1 & 2 & 3 & 4 & 5 & 6 & 7 & 8 & 9 & 10 & 11 & 12 & 13 & 14 & 15 & 16 \\
\hline$\overline{3}, 1$ & 6 & & & &.- & -..... & 1085.4 & (1066. 2) & 1051.28 & $(1039,53)$ & $(1029,69)$ & 1019.5 & 1007.3 & & \multirow{25}{*}{$\begin{array}{l}1317.6 \\
1317.6 \\
1326.14\end{array}$} & \multirow{25}{*}{$\begin{array}{l}(1296.7) \\
(1296.7)\end{array}$} & \multirow{25}{*}{$\begin{array}{l}1276.63 \\
1276.63\end{array}$} & \multirow{25}{*}{\begin{tabular}{l|l}
3 & 1255.95 \\
3 & 1255.95
\end{tabular}} \\
\hline$\overline{3}, 1$ & 4 & & & & - & 1180.75 & 1165.4 & 1151.59 & 1137.46 & 1120.9 & 1099.74 & 1072.69 & 1039.53 & & & & & \\
\hline$\overline{3}, 1$ & 2 & & & - & 1284.37 & 1265.42 & 1244.18 & 1218.63 & 1187.00 & 1149.48 & 1106.76 & 1060.14 & 1010.86 & 961.09 & & & & \\
\hline$\overline{3}, 1$ & 0 & & $\ldots$ & $(1379.6)$ & 1349. 39 & (1313.64) & 1271.80 & 1225.08 & 1174.54 & 1121.24 & 1066. 20 & 1010.12 & 953.49 & 896.65 & & & & \\
\hline$\underline{1}, \underline{1}$ & 1 & & 1498. 79 & 1464.92 & $(1423.9)$ & 1375.09 & 1320.09 & 1260.38 & 1198. 22 & 1135.80 & 1074.46 & 1014. 56 & 955.71 & 897.77 & & & & \\
\hline$\overline{1}, \overline{1}$ & 0 & (1557.5) & 1538.8 & 1522.67 & 1507 & 1490.81 & 1473.44 & 1455,26 & (1436. 7) & 1417.39 & 1397.74 & 1378.04 & 1358.06 & (1338.5) & & & & \\
\hline$\underline{1}, \overline{1}$ & 1 & & 1564.7 & 1540.1 & 1517.50 & 1496. 23 & 1476.29 & 1456.49 & (1436.7) & 1417.39 & 1397.74 & 1378.04 & 1358.06 & (1338.5) & & & & \\
\hline$\overline{1}, \underline{1}$ & 2 & & 1505.8 & 1487.34 & 1472.0 & 1459. 26 & 1447.91 & (1436. 7) & 1423.90 & 1409.94 & (1394. 5) & $(1378.0)$ & (1361, 1) & - & & & & \\
\hline $1, \overline{1}$ & 3 & & - . & $(1569.4)$ & $(1538.8)$ & 1503.55 & 1481.33 & $(1457.0)$ & 1435. 77 & 1416.11 & (1397.7) & 1379.63 & $(1362.7)$ & 1344.04 & & & & \\
\hline$\underline{1}, 1$ & 3 & & & 1457.09 & 1429. 97 & 1399.16 & 1362.70 & 1318.97 & 1268.40 & 1212.28 & 1152.44 & 1091.24 & 1030.58 & $(970.66)$ & & & & \\
\hline$\overline{1}, \overline{1}$ & 4 & & $\ldots$ & 1458. 24 & 1436.72 & $(1419.3)$ & 1404.98 & $(1394.5)$ & 1386.51 & 1379.63 & 1372.28 & $(1363.2)$ & 1352.41 & (1340) & & & & \\
\hline $1, \overline{1}$ & 5 & & & & 1591.9 & 1558.5 & $(1522.7)$ & 1489.23 & $(1457.1)$ & 1428.31 & 1403. 54 & 1382.11 & $(1362.7)$ & 1345.51 & & & & \\
\hline$\underline{1}, 1$ & 5 & & & & 1419.3 & 1394.50 & 1368.60 & 1340.35 & 1308.25 & 1269.97 & $(1225.0)$ & 1173.76 & 1117.71 & 1058.69 & & & & \\
\hline$\overline{1}, 1$ & 6 & & & & 1419.3 & 1395.81 & 1373.76 & 1354.87 & $(1340.3)$ & 1329.90 & 1323.31 & $(1319.0)$ & 1316.20 & & & & & \\
\hline$\underline{1}, 1$ & 7 & & & & & 1387.55 & 1363.17 & 1338. 56 & 1313.64 & 1287.38 & 1258.63 & 1225.5 & $(1187.0)$ & 1141.64 & & & & \\
\hline$\overline{1}, \overline{1}$ & 8 & & & & & 1387.55 & 1363.17 & 1339.55 & 1317.04 & 1296.67 & 1280.09 & $(1268,4)$ & 1257.07 & & & & & \\
\hline$\overline{1}, 1$ & 9 & & & & & 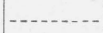 & 1361.09 & 1336.64 & 1312.61 & 1288. 28 & 1264.04 & 1239. 25 & 1213.0 & 1184.14 & & & & \\
\hline$\overline{1}, \overline{1}$ & 10 & & & & & - & 1361.09 & 1336.64 & 1312.61 & 1288. 92 & 1266.11 & 1244. 77 & 1226.1 & & & & & \\
\hline$\underline{1}, 1$ & 11 & & & & & & & 1339.23 & 1314.82 & 1290.59 & 1266.63 & 1242.90 & 1219.1 & 1195.41 & & & & \\
\hline$\overline{1}, \overline{1}$ & 12 & & & & & & $\ldots$ & 1339. 23 & 1314.82 & 1290.59 & 1266.63 & 1242.90 & 1220.43 & & & & & \\
\hline 1,1 & 13 & \} & & & & & & & 1320.90 & $(1296.67)$ & 1272,37 & 1248. 52 & $(1225.08)$ & 1201.55 & & & & \\
\hline 1,1 & 14 & \} & & & & & & & & $(1200.00)$ & 120.20 & 1270. 0 & $(1220.00)$ & 1201.00 & & & & \\
\hline$\frac{1}{1}, \frac{1}{1}$ & $\begin{array}{l}15 \\
16\end{array}$ & & & & & & & & & 1305.60 & 1281.22 & $(1257.1)$ & 1233.31 & 1209.79 & & & & \\
\hline$\overline{1}, 1$ & 17 & & & & & & & & & & & & & & & & & \\
\hline$\overline{1} \overline{1}$ & 18 & & & & & & & & & & 1292.40 & $(1200.4)$ & & & & & & \\
\hline
\end{tabular}

TABLE 4. Lines in the $R_{3, \overline{1}}, R_{3, \overline{3}}, R_{5,3}$, and $R_{5, \overline{5}}$ branches, pure rotation

\begin{tabular}{|c|c|c|c|c|c|c|c|c|c|c|c|c|c|}
\hline Branch & $(J+\tau)^{\prime \prime}$ & $J^{\prime \prime}=6$ & 7 & 8 & 9 & 10 & 11 & 12 & 13 & 14 & 15 & 16 & 17 \\
\hline $\begin{array}{l}3, \overline{1} \\
3, \overline{3}\end{array}$ & 0 & $\begin{array}{l}370.16 \\
484.05\end{array}$ & $\begin{array}{l}419.98 \\
545,55\end{array}$ & $\begin{array}{r}472.54 \\
(616.4)\end{array}$ & $\begin{array}{r}526.08 \\
(696.7)\end{array}$ & $\begin{array}{l}580.7 \\
784.54\end{array}$ & $\begin{array}{l}635.3 \\
87861\end{array}$ & $\begin{array}{c}(690.3) \\
976.07\end{array}$ & (744.1) & 799. 11 & 852.91 & 906.32 & 959.33 \\
\hline $\begin{array}{l}3, \overline{3} \\
3, \overline{1}\end{array}$ & $\begin{array}{l}1 \\
2\end{array}$ & $\begin{array}{l}484.05 \\
385.06\end{array}$ & $\begin{array}{l}545.55 \\
418.57\end{array}$ & $\begin{array}{l}(616.4) \\
(457.9)\end{array}$ & $\begin{array}{r}(696.7) \\
502.31\end{array}$ & $\begin{array}{l}784.54 \\
550.18\end{array}$ & $\begin{array}{l}878.61 \\
599.82\end{array}$ & $\begin{array}{r}976.07 \\
(650.5)\end{array}$ & $(703.0)$ & $(756)$ & 808.14 & 859,78 & 910.77 \\
\hline $3, \frac{1}{3}$ & 3 & $\begin{array}{l}506.96 \\
506\end{array}$ & 546.50 & 592.0 & $(644,7)$ & $(705.5)$ & 775.63 & 854.66 & 941.12 & & & & $90 . .1$ \\
\hline $3, \overline{1}$ & 4 & $(457.0)$ & $(472.5)$ & 492.08 & 517.00 & 547.88 & 574. 74 & $(625.3)$ & $(672.5)$ & (726) & $(784.54)$ & 840.36 & \\
\hline $3, \overline{3}$ & 5 & 566.9 & 594.5 & 625.25 & $(659.2)$ & (696.6) & $(742.6)$ & 794. 01 & 853.41 & 921.48 & & & \\
\hline $3, \overline{1}$ & 6 & 554. 82 & 569.2 & $(580.7)$ & $(592.0)$ & 605.0 & 620.7 & (641. 4) & $(669.6)$ & $(707)$ & & & \\
\hline $3, \overline{3}$ & 7 & $(638.1)$ & $(663.0)$ & $(688.0)$ & $(713.5)$ & $(740.7)$ & 770.17 & 803.60 & 841.16 & 883.18 & & & \\
\hline $3, \overline{1}$ & 8 & $(637.2)$ & $(659.4)$ & $(678.9)$ & $(694.0)$ & $(705,2)$ & $(713,0)$ & $(721.5)$ & $(730.8)$ & $(741)$ & & & \\
\hline $3, \overline{3}$ & 9 & & $(729.4)$ & (754. 8) & 779. 36 & 803.09 & 827.80 & $(852,62)$ & 878.9 & 906.8 & & & \\
\hline $3, \overline{1}$ & 10 & & $(729.2)$ & (754. 0) & 777.07 & 797.65 & 814,61 & 827.21 & 835.64 & $(841.1)$ & & & \\
\hline $3, \overline{3}$ & 11 & & & 813.97 & 840.01 & 865.51 & 890.14 & 914. 06 & 937.40 & 960.77 & & & \\
\hline $3, \overline{1}$ & 12 & & & 813.97 & 840.01 & 865.02 & 888.71 & 910.17 & 929.00 & & & & \\
\hline $3, \overline{3}$ & 13 & & & & 891.33 & 918,52 & 944.95 & 970.66 & & & & & \\
\hline $3, \frac{1}{2}$ & 14 & & & & & & & & & & & & \\
\hline $\begin{array}{l}3, \overline{3} \\
3, \overline{1}\end{array}$ & 15 & & & & & 962.05 & 990.3 & & & & & & \\
\hline $5, \overline{3}$ & 0 & & & & 798.75 & 871.36 & 948.35 & $(1028,5)$ & & & & & \\
\hline 5,3 & 2 & (673. 5) & $(707.4)$ & $(748.5)$ & 796.01 & 849.69 & 909.02 & 973. 59 & 1042.57 & & & & \\
\hline $5, \overline{3}$ & 4 & 792.2 & 808.33 & 827.80 & 852.62 & 883.89 & 922.19 & 967.00 & 1017.9 & & & & \\
\hline $5, \overline{3}$ & 6 & & 846.73 & 959.78 & 971.43 & 984.2 & 1000.35 & 1022.0 & & & & & \\
\hline $5, \overline{5}$ & 1 & 769.1 & 825.24 & 887.33 & 955.32 & 1029. 69 & & & & & & & \\
\hline $5, \overline{5}$ & 3 & 841.97 & 881.15 & 925.05 & 974.04 & 1028.47 & 1088.1 & & & & & & \\
\hline
\end{tabular}

TABLE 5. Rotational constants for $\nu_{2}$ of $\mathrm{H}_{2} \mathrm{O}$

\begin{tabular}{|c|c|c|c|c|c|c|}
\hline \multirow{2}{*}{$v_{1} v_{2} v_{3}$} & \multicolumn{6}{|c|}{ Constant } \\
\hline & A & $B$ & C & $D_{A}$ & $D_{B}$ & $D_{C}$ \\
\hline $\begin{array}{l}010- \\
000\end{array}$ & $\begin{array}{c}c m-1 \\
31.12 \\
27.877\end{array}$ & $\begin{array}{l}c m-1 \\
14.66 \\
14.512\end{array}$ & $\begin{array}{l}c m^{-1} \\
9.15 \\
9.285\end{array}$ & $\begin{array}{c}10^{-4} \mathrm{~cm}^{-1} \\
56.7 \\
33.7\end{array}$ & $\begin{array}{c}1 \mathrm{C}^{-4} \mathrm{~cm}^{-1} \\
2.6 \\
2.39\end{array}$ & $\begin{array}{c}10^{-4} \mathrm{~cm}^{-1} \\
0.4 \\
.25\end{array}$ \\
\hline
\end{tabular}

Washington, November 9, 1951. 